\title{
Multigene phylogeny of the family Cordycipitaceae (Hypocreales): new taxa and the new systematic position of the Chinese cordycipitoid fungus Paecilomyces hepiali
}

\author{
Yuan-Bing Wang ${ }^{1,2,3} \cdot$ Yao Wang ${ }^{1,2,4} \cdot$ Qi Fan ${ }^{1,2} \cdot$ Dong-E Duan ${ }^{1,2} \cdot$ Guo-Dong Zhang ${ }^{1,2,3} \cdot$ Ru-Qin Dai ${ }^{6}$. \\ Yong-Dong Dai ${ }^{1,2,4} \cdot$ Wen-Bo Zeng $^{7} \cdot \mathrm{Zi}$-Hong Chen ${ }^{8} \cdot$ Dan-Dan $\mathrm{Li}^{1,2} \cdot$ De-Xiang Tang ${ }^{1,2} \cdot$ Zhi-Hong Xu $^{1,2} \cdot$ Tao Sun $^{1,2,4}$. \\ Thi-Tra Nguyen ${ }^{2}$. Ngoc-Lan Tran ${ }^{9}$. Van-Minh Dao ${ }^{9}$. Can-Ming Zhang ${ }^{10}$ • Luo-Dong Huang ${ }^{1}$. Yong-Jun Liu ${ }^{11}$. \\ Xiao-Mei Zhang ${ }^{1,2,3,12} \cdot$ Da-Rong Yang ${ }^{13} \cdot$ Tatiana Sanjuan $^{14} \cdot$ Xing-Zhong Liu ${ }^{15} \cdot$ Zhu L. Yang $^{4,5} \cdot$ Hong Yu $^{1,2}$
}

Received: 15 November 2019 / Accepted: 31 July 2020 / Published online: 26 August 2020

(c) The Author(s) 2020

\begin{abstract}
The phylogeny and systematics of cordycipitoid fungi have been extensively studied in the last two decades. However, systematic positions of some taxa in the family Cordycipitaceae have not yet been thoroughly resolved. In this study, a new phylogenetic framework of Cordycipitaceae is reconstructed using multigene ( $\operatorname{nrSSU}, \mathrm{nr} L S U, t e f-1 \alpha, r p b 1$ and $r p b 2)$ sequence data with large-scale taxon sampling. In addition, ITS sequence data of species belonging to the Lecanicillium lineage in the family Cordycipitaceae are used to further determine their phylogenetic placements. Based on molecular phylogenetic data together with morphological evidence, two new genera (Flavocillium and Liangia), 16 new species and four new combinations are introduced. In the new genus Flavocillium, one new species $F$. bifurcatum and three new combinations previously described as Lecanicillium, namely F. acerosium, F. primulinium and F. subprimulinium, are proposed. The genus Liangia is built by the new species Lia. sinensis with Lecanicillium-like asexual morph, isolated from an entomopathogenic fungus Beauveria yunnanensis. Due to the absence of Paecilomyces hepiali, an economically and medically significant fungus, in the earlier phylogenetic analyses, its systematic position has been puzzling in both business and academic communities for a long time. Here, $P$. hepiali is recharacterized using the holotype material along with seven additional samples. It is assigned to the genus Samsoniella (Cordycipitaceae, Hypocreales) possessing Cordyceps-like sexual morph and Isaria-like asexual morph, and thus a new combination, namely S. hepiali is proposed. An additional nine new species in Samsoniella are described: S. alpina, S. antleroides, S. cardinalis, S. cristata, S. lanmaoa, S. kunmingensis, S. ramosa, S. tortricidae and S. yunnanensis. Four new species in Cordyceps are described: C. chaetoclavata, C. cocoonihabita, C. shuifuensis and C. subtenuipes. Simplicillium yunnanense, isolated from synnemata of Akanthomyces waltergamsii, is described as a new species.
\end{abstract}

Keywords Cordycipitaceae $\cdot$ Cordyceps $\cdot$ Flavocillium $\cdot$ Liangia $\cdot$ Paecilomyces hepiali $\cdot$ Phylogeny $\cdot$ Samsoniella

\section{Introduction}

In the taxonomic system of the twentieth century, Cordyceps Fr. sensu lato belonged to the family Clavicipitaceae s. 1 . characterized by possessing cylindrical asci, thickened ascus apices, and filiform ascospores that often disarticulate into

Yuan-Bing Wang, Yao Wang, Qi Fan, Dong-E Duan and GuoDong Zhang have equally contributed to this work.

Zhu L. Yang

fungi@mail.kib.ac.cn

Hong Yu

hongyu@ynu.edu.cn

Extended author information available on the last page of the article secondary ascospores (Mains 1958; Kobayasi 1982; Rossman et al. 1999, 2002; Sung et al. 2007). This genus is the most diverse group of Clavicipitaceae s. 1 . due to the large number of species and wide host range. The host associations for Cordyceps s. 1. are complex and diverse. Most of species are pathogens of more than 10 orders of invertebrates, while others are parasites of hypocrealean fungi, 
the truffle-like Elaphomyces Nees and myxomycetes (Kobayasi and Shimizu 1960; Kobayasi 1982; Sung et al. 2007; Kepler et al. 2013, 2017; Wang et al. 2015a,b). Phylogenetic analyses have indicated that neither Cordyceps s. 1. nor Clavicipitaceae s. 1. are monophyletic (Sung et al. 2007). Three cordycipitoid families are now recognized in the order Hypocreales: Clavicipitaceae, Cordycipitaceae and Ophiocordycipitaceae. At least 39 genera accommodating more than 1300 cordycipitoid species have been assigned to these three families (Sung et al. 2007; Chaverri et al. 2008; Johnson et al. 2009; Luangsa-ard et al. 2011, 2017; Kepler et al. 2013, 2014, 2017; Quandt et al. 2014; Spatafora et al. 2015; Tsang et al. 2016; Zare and Gams 2016; Mongkolsamrit et al. 2018).

The family Cordycipitaceae shares a common ancestor with Hypocreaceae and contains most of the species that have pallid or brightly pigmented, fleshy stromata (Sung et al. 2007; Maharachchikumbura et al. 2015). However, some species are characterized by possessing reduced stipes or subiculate stromata on the host. This family is the most complex group in Hypocreales with its varied morphological characteristics and wide-ranging hosts. Some genera with sexual or asexual morphs, such as Akanthomyces Lebert, Beauveria Vuill., Cordyceps, Gibellula Cavara, Isaria Pers., Lecanicillium W. Gams \& Zare and Torrubiella Boud., present numerous taxonomical problems and exist competing names. Numerous species of Cordyceps are associated with genera described originally for asexual morphs, including Akanthomyces, Beauveria, Evlachovaea B.A. Borisov \& Tarasov, Isaria, Lecanicillium, Microhilum H.Y. Yip $\&$ A.C. Rath and Paecilomyces Bainier. For example, in the genus Akanthomyces proposed by the type species $A$. aculeatus Lebert, C. tuberculata (Lebert) Maire is linked to an asexual morph A. pistillariiformis (Pat.) Samson \& H.C. Evans (Samson and Evans 1974). The sexual morph C. confragosa (Mains) G.H. Sung et al. described by Mains (1949) in Torrubiella, is linked to the type species Lecanicillium lecanii (Zimm.) Zare \& W. Gams of Lecanicillium and considered to be a synonym of Akanthomyces (Kepler et al. 2017). Cordyceps militaris (L.) Fr. also produces an asexual conidiogenous structure linked to Lecanicillium (Gams and Zare 2001). Cordyceps bassiana Z.Z. Li et al. was described as the sexual morph of B. bassiana (Bals.-Criv.) Vuill., the type species of Beauveria, which caused economically devastating epizootics of domestic larval silkworms in southern Europe during the eighteenth and nineteenth centuries (Li et al. 2001). Evlachovaea kintrischica B.A. Borisov \& Tarasov, the type species of Evlachovaea, was demonstrated to be a synonym of Isaria and was later combined into $C$. kintrischica (B.A. Borisov \& Tarasov) Kepler et al. (Humber et al. 2013; Kepler et al. 2017).

Kepler et al. (2017) provided the most complete taxonomic treatment of Cordycipitaceae and harmonized competing names based on principles of priority, recognition of monophyletic groups, and the practical usage of affected taxa, following Article 59 of the International Code of Nomenclature for algae, fungi and plants. They proposed to accommodate 11 genera within Cordycipitaceae, namely Akanthomyces, Ascopolyporus Möller, Beauveria, Blackwellomyces Spatafora \& Luangsa-ard, Cordyceps, Engyodontium de Hoog, Gibellula, Hyperdermium J.F. White et al., Hevansia Luangsa-ard et al., Parengyodontium C.C. Tsang et al. and Simplicillium W. Gams \& Zare. The other eight genera Evlachovaea, Granulomanus de Hoog \& Samson, Isaria, Lecanicillium, Microhilum, Phytocordyceps C.H. Su \& H.H. Wang, Synsterigmatocystis Costantin and Torrubiella were rejected. The genus Leptobacillium Zare \& W. Gams, recently described with L. leptobactrum (W. Gams) Zare \& W. Gams and two related new varieties, was added to the family Cordycipitaceae, presenting a sister generic relationship with Simplicillium (Zare and Gams 2016). The genus Amphichorda Fr. was established by Fries (1825) and comprised only one species, Amp. felina (DC.) Fr., which was later recombined into B. felina (DC.) J.W. Carmich. Recently, Zhang et al. (2017) described a species Amp. guana Z.F. Zhang, F. Liu \& L. Cai on bat guano in this genus based on multigene phylogeny and morphology. Subsequently, Mongkolsamrit et al. (2018) erected the genus Samsoniella Mongkols et al. to accommodate three species with orange cylindrical to clavate stromata, superficial perithecia and orange conidiophores with Isaria-like phialides and white to cream conidia, and to segregate them from the Akanthomyces group. Although several taxonomic studies have been conducted, many species originally described in Lecanicillium remain incertae sedis members in the family Cordycipitaceae and are polyphyletic (Zare and Gams 2016; Kepler et al. 2017; Mongkolsamrit et al. 2018). To date, 32 Lecanicillium species have been formally described and recorded in the Index Fungorum (https://www.indexfungo rum.org). Available data indicated that some species, such as $L$. aranearum (Petch) Zare \& W. Gams, L. antillanum (R.F. Castañeda \& G.R.W. Arnold) Zare \& W. Gams, $L$. primulinum Kaifuchi et al. and L. psalliotae (Treschew) Zare \& W. Gams represent basal to subbasal monophyletic clades in the family Cordycipitaceae (Kepler et al. 2017; Huang et al. 2018; Zhou et al. 2018). Therefore, new generic names for these species in the family Cordycipitaceae need to be introduced and supported by more detailed morphological and phylogenetic evidence combined with a larger taxon sampling.

The genus Paecilomyces erected by Bainier (1907), based on the type species $P$. variotii Bainier, was placed in the family Aspergillaceae (Eurotiales). Samson (1974) expanded Paecilomyces and recognized some mesophilic species previously placed in Isaria or Spicaria Harting as a distinguishing sect. Isarioidea with mostly insect hosts. However, a 
$\mathrm{nr} S S U$ phylogenetic analysis indicated that Paecilomyces is not monophyletic and the sect. Isarioidea is not a eurotialean lineage (Luangsa-ard et al. 2004). Based on the $\beta$-tubulin and ITS phylogentic data, Luangsa-ard et al. (2005) found that Paecilomyces sect. Isarioidea is polyphyletic in the order Hypocreales. The group designed as the Isaria clade is excluded from the genus Paecilomyces. It is monophyletic comprising of 10 Paecilomyces species, nine of which are subsequently transfered into Cordyceps.

Paecilomyces hepiali Q.T. Chen \& R.Q. Dai ex R.Q. Dai et al. was first isolated from natural Ophiocordyceps sinensis (Berk.) G.H. Sung et al. (syn. C. sinensis) associated with the larvae of Hepialus armoricanus Oberthür in China (Dai et al. 1989). This is a very important fungus because of its therapeutic benefits. However, molecular phylogenetic position of $P$. hepiali has been unclear for a long time due to the absence of nucleotide sequences from the holotype material. Recent phylogenetic analyses based on mitochondrial genomic sequences from five families within the order Hypocreales indicated that the putative P. hepiali specimen belongs to the family Cordycipitaceae ( $\mathrm{Li}$ et al. 2019). However, without any generic assignment, its wellestablished phylogenetic position within the family remains undetermined. For such a species that makes a significant contribution to human health, it is indispensable to elucidate its phylogeny and systematics using the holotype material.

During the last two decades, our efforts have been exerted in the investigation of cordycipitoid fungi especially in China and Southeast Asia. To date, over 18,000 specimens and 7500 strains of Cordyceps s. 1., representing more than 450 species, have been deposited in Yunnan University, Kunming, Yunnan Province. In this study, 1568 specimens and 1075 strains of Cordycipitaceae from different regions in Yunnan Province of China and Vietnam were analyzed using molecular phylogeny and morphology. Among these materials, five-gene (nrSSU, $\mathrm{nr} L S U$, tef- $1 \alpha, r p b 1$ and $r p b 2)$ data from 56 samples, and ITS data from two samples were selected and submitted to GenBank. We established phylogenetic and evolutionary trees by maximum likelihood (ML) and Bayesian inference (BI) analyses from the five-gene and ITS data. Two new genera, 16 new species and four new combinations are introduced.

\section{Materials and methods}

\section{Fungal materials and isolation}

The majority of Cordycipitaceae specimens were collected from Yunnan Province in China. Some specimens were collected from the Hoang Lien Mountains of Lao Cai Province in Vietnam. Specimens were noted and photographed in the fields. Collections were placed in sterilized plastic tubes and boxes, returned to the laboratory, and stored at $4{ }^{\circ} \mathrm{C}$. The specimens were examined using an Olympus SZ61 stereomicroscope. To obtain axenic cultures, the stromata or synnemata were removed from insect bodies and divided into 5-10 segments, each $3 \mathrm{~mm}$ long. The segments were immersed in $30 \% \mathrm{H}_{2} \mathrm{O}_{2}$ for $30 \mathrm{~s}$ and then rinsed five times in sterilized water. After drying on sterilized filter paper, segments were inoculated onto potato dextrose agar (PDA: potato $200 \mathrm{~g} / \mathrm{L}$, dextrose $20 \mathrm{~g} / \mathrm{L}$, agar $20 \mathrm{~g} / \mathrm{L}$ ) plates. The conidia of cordycipitoid fungi at the conidial masses were picked up with an inoculating loop and spread on PDA plates containing $0.1 \mathrm{~g} / \mathrm{l}$ streptomycin and $0.05 \mathrm{~g} / \mathrm{l}$ tetracycline. To isolate the strains from the sexual morph, the stroma containing mature perithecia was placed over a PDA plate and care was taken that the stroma was above the PDA plate and did not touch the agar surface in an effort to discharge ascospores. Discharged ascospores were removed with a sterile needle from the agar and transferred onto a new PDA plate containing $0.1 \mathrm{~g} / \mathrm{l}$ streptomycin and $0.05 \mathrm{~g} / \mathrm{l}$ tetracycline. Pure cultures were transferred onto PDA plates and incubated in a culture room at $25{ }^{\circ} \mathrm{C}$. After isolation into pure cultures, they were transplanted to a PDA slant and stored at $4{ }^{\circ} \mathrm{C}$. Specimens were deposited in Yunnan Herbal Herbarium (YHH) of Yunnan University. The cultures were deposited in Yunnan Fungal Culture Collection (YFCC) of Yunnan University.

\section{Morphological observations}

For descriptions of the sexual morph, fruiting bodies were photographed and measured using an Olympus SZ61 stereomicroscope. Hand sections of the fruiting structures were mounted in water or lactophenol cotton blue solution for microscopic studies and photomicrography. The micromorphological characteristics of fungi such as perithecia, asci and ascospores were examined using Olympus CX40 and BX53 microscopes. Cultures on slants were transferred to PDA plates and cultured in an incubator for 21 days at $25{ }^{\circ} \mathrm{C}$. The circular agar blocks, circa $5 \mathrm{~mm}$ in diameter, from a colony were removed and placed on new PDA plates to observe colony morphology. Colonies were photographed and measured every fourth day. For asexual morphological descriptions, microscope slide cultures were prepared by placing a small amount of mycelia on $5-\mathrm{mm}$ diameter PDA medium blocks overlaid by a cover slip. Micro-morphological observations and measurements were conducted using Olympus CX40 and BX53 microscopes, and a FEI QUANTA200 scanning electron microscope.

\section{DNA extraction, PCR, and sequencing}

Clean-washed specimens and axenic living cultures were prepared for DNA extraction. Total DNA was extracted using the CTAB method described by Liu et al. (2001). The 
following primer pairs were used for PCR amplification. The primer pair, $\mathrm{nrSSU}-\mathrm{CoF}$ and $\mathrm{nrSSU}$-CoR was used to amplify the nuclear ribosomal small subunit (nrSSU) (Wang et al. 2015a). The primer pair, LR5 and LR0R was used to amplify the nuclear ribosomal large subunit ( $\mathrm{nr} L S U$ ) (Vilgalys and Hester 1990; Rehner and Samuels 1994). The primer pair, EF $1 \alpha-\mathrm{EF}$ and $\mathrm{EF} 1 \alpha-\mathrm{ER}$ was used to amplify the translation elongation factor $1 \alpha(t e f-1 \alpha)$ (Bischoff et al. 2006; Sung et al. 2007). The two primer pairs, RPB1-5'F and RPB1-5'R, RPB2-5'F and RPB2-5'R were used to amplify the largest and second largest subunits of RNA polymerase II (rpbland rpb2), respectively (Bischoff et al. 2006; Sung et al. 2007). The primer pair, ITS4 and ITS5 was used to amplify the nuclear ribosomal internal transcribed spacer region (ITS) (White et al. 1990). Polymerase chain reaction (PCR) assays of five genes and ITS were performed as previously described (Bischoff et al. 2006; Wang et al. 2015b). The PCR assay was conducted as described by Wang et al. (2015b). PCR products were separated by electrophoresis in 1.0\% agarose gels, purified using the Gel Band Purification Kit (Bio Teke Co., Ltd, Beijing, China) and then sequenced on an automatic sequence analyser (BGI Co., Ltd, Shenzhen, China). When PCR products could not be sequenced directly, coloning was performed by the TaKaRa $\mathrm{PMD}^{\mathrm{TM}} 18-\mathrm{T}$ vector system (TaKaRa Biotechnology Co., Ltd, Dalian, China).

\section{Phylogenetic analyses}

Five-gene (nrSSU, $\mathrm{nr} L S U$, tef-1 $\alpha, r p b 1$ and $r p b 2)$ sequences from 56 samples of 30 species belonging to six genera, and ITS sequences from two samples of the new species Flavocillium bifurcatum, were newly generated. Sequences of five genes and ITS were retrieved from GenBank, and then combined with the newly generated sequences. The taxon information and GenBank accession numbers of five genes were listed in Table 1. GenBank accession numbers of ITS sequences were placed in the front of the species name, appearing in the ITS phylogenetic tree (Fig. 3). Sequences of five genes and ITS were aligned using Clustal X2.0 and MEGA6 (Larkin 2007; Tamura et al. 2013). Ambiguously aligned sites were excluded from phylogenetic analyses, and gaps were treated as missing data. Adjustment to the computer-assisted alignment was necessary regarding the nrSSU sequences containing an intron. These sequences were manually adjusted and ambiguous regions created by insertions and deletions (indel) were eliminated. After sequence alignments, the aligned sequences of five genes were concatenated. Conflicts between the five genes were tested using PAUP* 4.0b10 (Swofford 2002). The results showed that the phylogenetic signals in the five genes were not in conflict. Eleven data partitions were defined for the combined five-gene dataset employing PartitionFinder V1.1.1 (Lanfear et al. 2012). These included one each for $\operatorname{nr} S S U$ and $\operatorname{nr} L S U$, and three for each of the three codon positions of tef- $1 \alpha$, $r p b 1$ and $r p b 2$. Phylogenetic analyses of the five-gene and ITS datasets were conducted using ML and BI methods. ML analyses were performed with RAxML v7.9.1 using the optimal model GTR + I with 1000 rapid bootstrap replicates on the five-gene and ITS datasets (Stamatakis 2006). The model was separately applied to each of the 11 partitions of five genes. BI analyses were performed with MrBayes v3.1.2 for five million generations using a GTR $+\mathrm{G}+\mathrm{I}$ model determined by jModelTest version 2.1.4 and employed the model separately for each partition of five-gene analyses, whereas the default F81 model was used for the ITS analyses (Ronquist and Huelsenbeck 2003; Darriba et al. 2012). Trees were sampled every 100 generations. The first $25 \%$ trees were discarded as burn-in and the remaining trees were used to create a consensus tree using the sumt demand. Phylogenetic trees were visualised and modified using the Interactive Tree Of Life (iTOL) (https://itol.embl.de) online tool (Letunic and Bork 2019).

\section{Results}

In ML and BI phylogenetic analyses, five-gene sequences of 30 species collected in this study were employed to reconstruct phylogenetic framework of the family Cordycipitaceae. Taxa within the order Hypocreales consisted of four families, viz. Cordycipitaceae, Ophicordycipitaceae, Clavicipitaceae, Hypocreaceae, and two taxa of Nectriaceae (Nectria cinnabarina CBS 114055 and Gliocephalotrichum bulbilium ATCC 22228) designated as the outgroup. The concatenated sequence dataset of 241 taxa was composed of $4837 \mathrm{bp}$ sequence data (1082 bp for nrSSU, $904 \mathrm{bp}$ for $\mathrm{nr} L S U, 1064 \mathrm{bp}$ for $t e f-1 \alpha, 802 \mathrm{bp}$ for $r p b 1$ and $985 \mathrm{bp}$ for $r p b 2)$. Phylogenetic trees obtained from ML and BI analyses were identical in overall topologies and were not significantly different (Fig. 1). Most well-resolved genera and lineages in Cordycipitaceae shared similar relationships with previous analyses (Sung et al. 2007; Sukarno et al. 2009; Kepler et al. 2017; Mongkolsamrit et al. 2018). Our ML and BI analyses showed that the placement of Cordycipitaceae in the order Hypocreales was well-supported by bootstrap proportions $(\mathrm{BP}=70 \%)$ and posterior probabilities $(\mathrm{PP}=95 \%)$, respectively.

Species in the typifed genus Lecanicillium were distributed throughout the family Cordycipitaceae and were polyphyletic (Fig. 1,2). These species were clustered into the clades of $L$. aranearum, $L$. antillanum, $L$. primulinum, $L$. fusisporum and $L$. psalliotae, respectively. In the five-gene phylogenetic tree, the $L$. primulinum clade harbored $L$. primulinum, $L$. acerosum W. Gams et al., Lecanicillium sp. and another new species (YFCC 6101) described in this study. ML and BI phylogenetic 
Table 1 Specimen information and GenBank accession numbers for sequences used in this study

\begin{tabular}{|c|c|c|c|c|c|c|}
\hline \multirow[t]{2}{*}{ Taxon } & \multirow[t]{2}{*}{ Voucher information } & \multicolumn{5}{|c|}{ GenBank accession number } \\
\hline & & $\operatorname{nrSSU}$ & $\operatorname{nr} L S U$ & tef- $1 \alpha$ & $r p b 1$ & $r p b 2$ \\
\hline Akanthomyces aculeatus & HUA 186145 & MF416572 & MF416520 & MF416465 & & \\
\hline Akanthomyces aculeatus & HUA 772 & KC519368 & KC519370 & KC519366 & & \\
\hline Akanthomyces attenuatus & CBS 402.78 & AF339614 & AF339565 & EF468782 & EF468888 & EF468935 \\
\hline Akanthomyces cf. coccidioperitheciatus & NHJ 5112 & EU369109 & EU369043 & EU369026 & EU369066 & \\
\hline Akanthomyces coccidioperitheciatus & NHJ 6709 & EU369110 & EU369042 & EU369025 & EU369067 & EU369086 \\
\hline Akanthomyces dipterigenus & CBS 126.27 & AF339605 & AF339556 & KM283820 & KR064300 & KR064303 \\
\hline Akanthomyces kanyawimiae & TBRC 7242 & & MF140718 & MF140838 & MF140784 & MF140808 \\
\hline Akanthomyces kanyawimiae & TBRC 7244 & & MF140716 & MF140836 & & \\
\hline Akanthomyces lecanii & CBS 101247 & AF339604 & AF339555 & DQ522359 & DQ522407 & DQ522466 \\
\hline Akanthomyces muscarius & CBS 143.62 & KM283774 & KM283798 & KM283821 & KM283841 & KM283863 \\
\hline Akanthomyces pistillariaeformis & HUA 186131 & MF416573 & MF416521 & MF416466 & & \\
\hline Akanthomyces sabanensis & ANDES-F 1023 & KC633253 & & KC633267 & KC875222 & \\
\hline Akanthomyces sabanensis & ANDES-F 1024 & KC633251 & KC875225 & KC633266 & & KC633249 \\
\hline Akanthomyces sulphureus & TBRC 7247 & & MF140720 & MF140841 & MF140785 & MF140811 \\
\hline Akanthomyces sulphureus & TBRC 7248 & & MF140722 & MF140843 & MF140787 & MF140812 \\
\hline Akanthomyces thailandicus & TBRC 7245 & & & MF140839 & & MF140809 \\
\hline Akanthomyces thailandicus & TBRC 7246 & & MF140719 & MF140840 & & MF140810 \\
\hline Akanthomyces tuberculatus & OSC 111002 & DQ522553 & DQ518767 & DQ522338 & DQ522384 & DQ522435 \\
\hline Akanthomyces tuberculatus & BCC 16819 & MF416600 & MF416546 & MF416490 & MF416647 & MF416444 \\
\hline Akanthomyces waltergamsii & TBRC 7251 & & MF140713 & MF140833 & MF140781 & MF140805 \\
\hline Akanthomyces waltergamsii & TBRC 7252 & & MF140714 & MF140834 & MF140782 & MF140806 \\
\hline Amphichorda guana & CGMCC 3.17908 & & KU746711 & KX855211 & & \\
\hline Ascopolyporus polychrous & P.C. 546 & & DQ118737 & DQ118745 & DQ127236 & \\
\hline Ascopolyporus villosus & ARSEF 6355 & & AY886544 & DQ118750 & DQ127241 & \\
\hline Beauveria acridophila & HUA 179220 & JQ895527 & JQ895536 & JQ958614 & JX003852 & JX003842 \\
\hline Beauveria acridophila & HUA 179219 & & JQ895541 & JQ958613 & JX003857 & JX003841 \\
\hline Beauveria amorpha & ARSEF 2641 & & AB100039 & AY531917 & HQ880880 & HQ880952 \\
\hline Beauveria araneola & GZAC 150317 & & & KT961699 & KT961701 & \\
\hline Beauveria asiatica & ARSEF 4850 & & & AY531937 & HQ880859 & HQ880931 \\
\hline Beauveria asiatica & YFCC 5600 & MN576770 & MN576826 & MN576996 & MN576886 & MN576940 \\
\hline Beauveria australis & ARSEF 4598 & & & HQ880995 & HQ880861 & HQ880933 \\
\hline Beauveria bassiana & ARSEF 1564 & EU334676 & & HQ880974 & HQ880833 & HQ880905 \\
\hline Beauveria bassiana & YFCC 3369 & MN576768 & MN576824 & MN576994 & MN576884 & MN576938 \\
\hline Beauveria blattidicola & MCA 1727 & MF416593 & MF416539 & MF416483 & MF416640 & \\
\hline Beauveria blattidicola & MCA 1814 & MF416594 & MF416540 & MF416484 & MF416641 & \\
\hline Beauveria brongniartii & YFCC 3240 & MN576769 & MN576825 & MN576995 & MN576885 & MN576939 \\
\hline Beauveria brongniartii & ARSEF 617 & AB027335 & AB027381 & HQ880991 & HQ880854 & HQ880926 \\
\hline Beauveria caledonica & ARSEF 2567 & AF339570 & AF339520 & EF469057 & HQ880889 & HQ880961 \\
\hline Beauveria caledonica & YFCC 7025 & MN576771 & MN576827 & MN576997 & MN576887 & MN576941 \\
\hline Beauveria diapheromeriphila & QCNE 186272 & JQ895530 & JQ895534 & JQ958610 & JX003848 & \\
\hline Beauveria diapheromeriphila & QCNE 186714 & MF416601 & MF416547 & MF416491 & MF416648 & \\
\hline Beauveria hoplocheli & MNHN-RF-06107 & & & KC339702 & KM453954 & KM453963 \\
\hline Beauveria hoplocheli & Bt116 & & & KC339703 & KM453957 & KM453966 \\
\hline Beauveria kipukae & ARSEF 7032 & & & HQ881005 & HQ880875 & HQ880947 \\
\hline Beauveria locustiphila & HUA 179217 & JQ958598 & JQ958597 & & JX003847 & \\
\hline Beauveria locustiphila & HUA 179218 & JQ895525 & JQ895535 & JQ958619 & JX003846 & JX003845 \\
\hline Beauveria malawiensis & ARSEF 7760 & & & DQ376246 & HQ880897 & HQ880969 \\
\hline Beauveria pseudobassiana & YFCC 7120 & MN576772 & MN576828 & MN576998 & MN576888 & MN576942 \\
\hline
\end{tabular}


Table 1 (continued)

\begin{tabular}{|c|c|c|c|c|c|c|}
\hline \multirow[t]{2}{*}{ Taxon } & \multirow[t]{2}{*}{ Voucher information } & \multicolumn{5}{|c|}{ GenBank accession number } \\
\hline & & $\mathrm{nrSSU}$ & $\operatorname{nr} L S U$ & tef- $1 \alpha$ & $r p b 1$ & $r p b 2$ \\
\hline Beauveria pseudobassiana & ARSEF 3405 & & & AY531931 & HQ880864 & HQ880936 \\
\hline Beauveria scarabaeidicola & ARSEF 5689 & AF339574 & AF339524 & DQ522335 & DQ522380 & DQ522431 \\
\hline Beauveria staphylinidicola & ARSEF 5718 & EF468981 & EF468836 & EF468776 & EF468881 & \\
\hline Beauveria varroae & ARSEF 8257 & & & HQ881002 & HQ880872 & HQ880944 \\
\hline Beauveria vermiconia & ARSEF 2922 & & & AY531920 & HQ880894 & HQ880966 \\
\hline Beauveria yunnanensis & YFCC 3105 & MN576773 & MN576829 & MN576999 & MN576889 & MN576943 \\
\hline Blackwellomyces cardinalis & OSC 93609 & AY184973 & AY184962 & DQ522325 & DQ522370 & DQ522422 \\
\hline Blackwellomyces cardinalis & OSC 93610 & AY184974 & AY184963 & EF469059 & EF469088 & EF469106 \\
\hline Blackwellomyces pseudomilitaris & BCC 1919 & MF416588 & MF416534 & MF416478 & & MF416440 \\
\hline Blackwellomyces pseudomilitaris & BCC 2091 & MF416589 & MF416535 & MF416479 & & MF416441 \\
\hline Claviceps purpurea & S.A. cp11 & EF469122 & EF469075 & EF469058 & EF469087 & EF469105 \\
\hline Cordyceps albocitrina & spat $07-174$ & MF416575 & & MF416467 & MF416629 & \\
\hline Cordyceps amoenerosea & CBS 107.73 & AY526464 & MF416550 & MF416494 & MF416651 & MF416445 \\
\hline Cordyceps amoenerosea & CBS 729.73 & MF416604 & MF416551 & MF416495 & MF416652 & MF416446 \\
\hline Cordyceps bifusispora & spat $08-129$ & MF416576 & MF416523 & MF416468 & MF416630 & \\
\hline Cordyceps bifusispora & spat $08-133.1$ & MF416577 & MF416524 & MF416469 & MF416631 & MF416434 \\
\hline Cordyceps bifusispora & EFCC 5690 & EF468952 & EF468806 & EF468746 & EF468854 & EF468909 \\
\hline Cordyceps bifusispora & EFCC 8260 & EF468953 & EF468807 & EF468747 & EF468855 & EF468910 \\
\hline Cordyceps blackwelliae & TBRC 7255 & & MF140703 & MF140823 & MF140772 & MF140796 \\
\hline Cordyceps blackwelliae & TBRC 7256 & & MF140702 & MF140822 & MF140771 & MF140795 \\
\hline Cordyceps caloceroides & MCA 2249 & MF416578 & MF416525 & MF416470 & MF416632 & \\
\hline Cordyceps cateniannulata & CBS 152.83 & AY526465 & MG665226 & JQ425687 & & \\
\hline Cordyceps cateniobliqua & YFCC 3367 & MN576765 & MN576821 & MN576991 & MN576881 & MN576935 \\
\hline Cordyceps cateniobliqua & YFCC 5935 & MN576766 & MN576822 & MN576992 & MN576882 & MN576936 \\
\hline Cordyceps cateniobliqua & CBS 153.83 & AY526466 & & JQ425688 & & MG665236 \\
\hline Cordyceps cf. ochraceostromata & ARSEF 5691 & EF468964 & EF468819 & EF468759 & EF468867 & EF468921 \\
\hline Cordyceps cf. pruinosa & NHJ 10627 & EF468967 & EF468822 & EF468763 & EF468870 & \\
\hline Cordyceps cf. pruinosa & NHJ 10684 & EF468968 & EF468823 & EF468761 & EF468871 & \\
\hline Cordyceps cf. pruinosa & EFCC 5693 & EF468966 & EF468821 & EF468762 & EF468869 & \\
\hline Cordyceps cf. pruinosa & EFCC 5197 & EF468965 & EF468820 & EF468760 & EF468868 & \\
\hline Cordyceps cf. takaomontana & BCC 12688 & MF416599 & MF416545 & MF416489 & MF416646 & \\
\hline Cordyceps cf. takaomontana & NHJ 12623 & EF468984 & EF468838 & EF468778 & EF468884 & EF468932 \\
\hline Cordyceps chaetoclavata & YHH 15101 & MN576722 & MN576778 & MN576948 & MN576838 & MN576894 \\
\hline Cordyceps cicadae & RCEF HP090724-31 & MF416605 & MF416552 & MF416496 & MF416653 & MF416447 \\
\hline Cordyceps cocoonihabita & YFCC 3415 & MN576723 & MN576779 & MN576949 & MN576839 & MN576895 \\
\hline Cordyceps cocoonihabita & YFCC 3416 & MN576724 & MN576780 & MN576950 & MN576840 & MN576896 \\
\hline Cordyceps coleopterorum & CBS 110.73 & JF415965 & JF415988 & JF416028 & JN049903 & JF416006 \\
\hline Cordyceps exasperata & MCA 2288 & MF416592 & MF416538 & MF416482 & MF416639 & \\
\hline Cordyceps farinosa & CBS 111113 & AY526474 & MF416554 & MF416499 & MF416656 & MF416450 \\
\hline Cordyceps fumosorosea & YFCC 4561 & MN576761 & MN576817 & MN576987 & MN576877 & MN576931 \\
\hline Cordyceps fumosorosea & CBS 244.31 & MF416609 & MF416557 & MF416503 & MF416660 & MF416454 \\
\hline Cordyceps fumosorosea & CBS 375.70 & & AB083035 & MF416501 & MF416658 & MF416452 \\
\hline Cordyceps fumosorosea & CBS 107.10 & & MG665227 & HM161735 & & MG665237 \\
\hline Cordyceps grylli & MFLU 17-1023 & MK863048 & MK863055 & MK860193 & & \\
\hline Cordyceps grylli & MFLU 17-1024 & MK863049 & MK863056 & MK860194 & & \\
\hline Cordyceps javanica & YFCC 3368 & MN576767 & MN576823 & MN576993 & MN576883 & MN576937 \\
\hline Cordyceps javanica & TBRC 7259 & & MF140711 & MF140831 & MF140780 & MF140804 \\
\hline Cordyceps javanica & CBS 134.22 & MF416610 & MF416558 & MF416504 & MF416661 & MF416455 \\
\hline
\end{tabular}


Table 1 (continued)

\begin{tabular}{|c|c|c|c|c|c|c|}
\hline \multirow[t]{2}{*}{ Taxon } & \multirow[t]{2}{*}{ Voucher information } & \multicolumn{5}{|c|}{ GenBank accession number } \\
\hline & & $\mathrm{nrSSU}$ & $\mathrm{nr} L S U$ & $t e f-1 \alpha$ & $r p b 1$ & $r p b 2$ \\
\hline Cordyceps kyusyuensis & EFCC 5886 & EF468960 & EF468813 & EF468754 & EF468863 & EF468917 \\
\hline Cordyceps militaris & YFCC 6587 & MN576762 & MN576818 & MN576988 & MN576878 & MN576932 \\
\hline Cordyceps militaris & YFCC 5840 & MN576763 & MN576819 & MN576989 & MN576879 & MN576933 \\
\hline Cordyceps morakotii & BCC 55820 & & MF140730 & KT261399 & & \\
\hline Cordyceps morakotii & BCC 68398 & & MF140731 & KT261398 & & \\
\hline Cordyceps ninchukispora & EGS 38.165 & EF468991 & EF468846 & EF468795 & EF468900 & \\
\hline Cordyceps ninchukispora & EGS 38.166 & EF468992 & EF468847 & EF468794 & EF468901 & \\
\hline Cordyceps ningxiaensis & HMJAU 25074 & & KF309671 & & & \\
\hline Cordyceps ningxiaensis & HMJAU 25076 & & KF309673 & & & \\
\hline Cordyceps oncoperae & ARSEF 4358 & AF339581 & AF339532 & EF468785 & EF468891 & EF468936 \\
\hline Cordyceps piperis & CBS 116719 & & AY466442 & DQ118749 & DQ127240 & EU369083 \\
\hline Cordyceps polyarthra & MCA 996 & MF416597 & MF416543 & MF416487 & MF416644 & \\
\hline Cordyceps polyarthra & MCA 1009 & MF416598 & MF416544 & MF416488 & MF416645 & \\
\hline Cordyceps pruinosa & ARSEF 5413 & AY184979 & AY184968 & DQ522351 & DQ522397 & DQ522451 \\
\hline Cordyceps rosea & spat $09-053$ & MF416590 & MF416536 & MF416480 & MF416637 & MF416442 \\
\hline Cordyceps roseostromata & ARSEF 4871 & AF339573 & AF339523 & & & \\
\hline Cordyceps shuifuensis & YFCC 5230 & MN576721 & MN576777 & MN576947 & MN576837 & MN576893 \\
\hline Cordyceps sp. & CBS 101284 & AF339613 & AF339564 & EF468803 & EF468907 & EF468948 \\
\hline Cordyceps sp. & EFCC 2535 & EF468980 & EF468835 & EF468772 & & \\
\hline Cordyceps sp. & YFCC 5833 & MN576764 & MN576820 & MN576990 & MN576880 & MN576934 \\
\hline Cordyceps spegazzinii & ARSF 7850 & & DQ196435 & & & \\
\hline Cordyceps subtenuipes & YFCC 6051 & MN576719 & MN576775 & MN576945 & MN576835 & MN576891 \\
\hline Cordyceps subtenuipes & YFCC 6084 & MN576720 & MN576776 & MN576946 & MN576836 & MN576892 \\
\hline Cordyceps succavus & MFLU 18-1890 & MK086058 & MK086062 & & MK084616 & MK079353 \\
\hline Cordyceps tenuipes & ARSEF 5135 & MF416612 & JF415980 & JF416020 & JN049896 & JF416000 \\
\hline Cordyceps tenuipes & YFCC 4266 & MN576774 & MN576830 & MN577000 & MN576890 & MN576944 \\
\hline Drechmeria gunnii & OSC 76404 & AF339572 & AF339522 & AY489616 & AY489650 & DQ522426 \\
\hline Engyodontium aranearum & CBS 309.85 & AF339576 & AF339526 & DQ522341 & DQ522387 & DQ522439 \\
\hline Engyodontium parvisporum & IHEM 22910 & & LC092915 & & & \\
\hline Engyodontium rectidentatum & CBS 206.74 & & LC092912 & & & \\
\hline Engyodontium rectidentatum & CBS 641.74 & & LC092914 & & & \\
\hline Flavocillium bifurcatum & YFCC 6101 & MN576725 & MN576781 & MN576951 & MN576841 & MN576897 \\
\hline Flavocillium primulinum & JCM 18525 & & AB712263 & & & \\
\hline Flavocillium primulinum & JCM 18526 & & AB712264 & & & \\
\hline Flavocillium primulinum & JCM 18527 & & AB712265 & & & \\
\hline Gibellula cf. alba & NHJ 11679 & & & EU369016 & EU369054 & \\
\hline Gibellula leiopus & BCC 16025 & MF416602 & MF416548 & MF416492 & MF416649 & \\
\hline Gibellula longispora & NHJ 12014 & EU369098 & & EU369017 & EU369055 & EU369075 \\
\hline Gibellula pulchra & NHJ 10808 & EU369099 & EU369035 & EU369018 & EU369056 & EU369076 \\
\hline Gibellula sp. & NHJ 10788 & EU369101 & EU369036 & EU369019 & EU369058 & EU369078 \\
\hline Gibellula sp. & NHJ 5401 & EU369102 & & & EU369059 & EU369079 \\
\hline Gibellula sp. & NHJ 13158 & EU369100 & EU369037 & EU369020 & EU369057 & EU369077 \\
\hline Gliocephalotrichum bulbilium & ATCC 22228 & AY489700 & AY489732 & AY489627 & AY489664 & EF469114 \\
\hline Harposporium harposporiferum & ARSEF 5472 & AF339569 & AF339519 & DQ118747 & DQ127238 & \\
\hline Hevansia arachnophilus & NHJ 10469 & EU369090 & EU369031 & EU369008 & EU369047 & \\
\hline Hevansia cinereus & NHJ 3510 & EU369091 & & EU369009 & EU369048 & EU369070 \\
\hline Hevansia nelumboides & BCC 41864 & JN201863 & JN201873 & JN201867 & & \\
\hline Hevansia novoguineensis & NHJ 13161 & EU369093 & & EU369011 & EU369050 & \\
\hline
\end{tabular}


Table 1 (continued)

\begin{tabular}{|c|c|c|c|c|c|c|}
\hline \multirow[t]{2}{*}{ Taxon } & \multirow[t]{2}{*}{ Voucher information } & \multicolumn{5}{|c|}{ GenBank accession number } \\
\hline & & $\mathrm{nrSSU}$ & $\operatorname{nr} L S U$ & $t e f-1 \alpha$ & $r p b 1$ & $r p b 2$ \\
\hline Hevansia novoguineensis & NHJ 13117 & EU369092 & & EU369010 & EU369049 & EU369073 \\
\hline Hevansia novoguineensis & NHJ 4314 & EU369094 & & EU369012 & EU369051 & EU369071 \\
\hline Hevansia novoguineensis & NHJ 11923 & EU369095 & EU369032 & EU369013 & EU369052 & EU369072 \\
\hline Hevansia sp. & BCC 28584 & GQ249965 & GQ249989 & GQ250040 & & \\
\hline Hyperdermium caulium & Genebank AF242354 & & AF242354 & & & \\
\hline Hyperdermium pulvinatum & P.C. 602 & & DQ118738 & DQ118746 & DQ127237 & \\
\hline Hypocrella siamensis & BCC 8105 & DQ522537 & DQ518752 & DQ522317 & DQ522363 & DQ522411 \\
\hline Isaria cf. farinosa & OSC 111004 & EF468986 & EF468840 & EF468780 & EF468886 & \\
\hline Isaria cicadae & YFCC 7128 & MN576759 & MN576815 & MN576985 & MN576875 & MN576929 \\
\hline Isaria cicadae & YFCC 7019 & MN576760 & MN576816 & MN576986 & MN576876 & MN576930 \\
\hline Isaria farinosa & OSC 111005 & DQ522558 & DQ518773 & DQ522348 & DQ522394 & \\
\hline Isaria farinosa & OSC 111006 & EF469127 & EF469080 & EF469065 & EF469094 & \\
\hline Isaria $\mathrm{sp}$. & spat $09-050$ & MF416613 & MF416559 & MF416506 & MF416663 & MF416457 \\
\hline Isaria sp. & spat $09-051$ & MF416614 & MF416560 & MF416507 & MF416664 & MF416458 \\
\hline Isaria sp. & TNS 16333 & MF416611 & & MF416505 & MF416662 & MF416456 \\
\hline Lecanicillium acerosum & CBS 418.81 & KM283762 & KM283786 & KM283810 & KM283832 & KM283852 \\
\hline Lecanicillium antillanum & CBS 350.85 & AF339585 & AF339536 & DQ522350 & DQ522396 & DQ522450 \\
\hline Lecanicillium aranearum & CBS 726.73a & AF339586 & AF339537 & EF468781 & EF468887 & EF468934 \\
\hline Lecanicillium fusisporum & CBS 164.70 & KM283769 & KM283793 & KM283817 & KM283836 & KM283858 \\
\hline Lecanicillium psalliotae & CBS 363.86 & AF339608 & AF339559 & EF468784 & EF468890 & \\
\hline Lecanicillium psalliotae & CBS 101270 & EF469128 & EF469081 & EF469066 & EF469095 & EF469113 \\
\hline Lecanicillium psalliotae & CBS 532.81 & AF339609 & AF339560 & EF469067 & EF469096 & EF469112 \\
\hline Lecanicillium $\mathrm{sp}$. & KYK00214 & & AB378528 & & & \\
\hline Lecanicillium $\mathrm{sp}$. & КYК00305 & & AB378529 & & & \\
\hline Lecanicillium sp. & CBS 639.85 & KM283777 & KM283801 & KM283824 & KM283843 & KM283865 \\
\hline Leptobacillium chinense & LC1345 & & JQ410322 & & & \\
\hline Leptobacillium coffeanum & CDA 734 & & MF066032 & & & \\
\hline Leptobacillium leptobactrum & CBS 771.69 & & KU382224 & & & \\
\hline Leptobacillium leptobactrum & IRAN 1230 & & KU382225 & & & \\
\hline $\begin{array}{l}\text { Leptobacillium leptobactrum var. } \\
\text { calidius }\end{array}$ & CBS 748.73 & & KU382227 & & & \\
\hline $\begin{array}{l}\text { Leptobacillium leptobactrum var. } \\
\text { calidius }\end{array}$ & CBS 703.86 & & KU382226 & & & \\
\hline Leptobacillium muralicola & CGMCC3.19014 & & MH379997 & & & \\
\hline Leptobacillium symbioticum & KYK00024 & & AB378539 & & & \\
\hline Leptobacillium symbioticum & Soy1-2 & & LC506046 & & & \\
\hline Leptobacillium symbioticum & OPTF00168 & & LC506047 & & & \\
\hline Liangia sinensis & YFCC 3103 & MN576726 & MN576782 & MN576952 & MN576842 & MN576898 \\
\hline Liangia sinensis & YFCC 3104 & MN576727 & MN576783 & MN576953 & MN576843 & MN576899 \\
\hline Metapochonia suchlasporia var. suchlasporia & CBS 251.83 & AF339615 & MH873311 & KJ398790 & KJ398601 & KJ398697 \\
\hline Metarhizium guizhouense & CBS 258.90 & MH143830 & MH873894 & EU248862 & EU248914 & EU248942 \\
\hline Nectria cinnabarina & CBS 114055 & U32412 & U00748 & AF543785 & AY489666 & DQ522456 \\
\hline Ophiocordyceps sinensis & EFCC 7287 & EF468971 & EF468827 & EF468767 & EF468874 & EF468924 \\
\hline Ophiocordyceps unilateralis & OSC 128574 & DQ522554 & DQ518768 & DQ522339 & DQ522385 & DQ522436 \\
\hline Parengyodontium album & CBS 504.83 & & LC092899 & & & \\
\hline Parengyodontium album & IHEM 4198 & JF797223 & LC092906 & DQ268655 & & \\
\hline Parengyodontium album & CBS 368.72 & & LC092910 & & & \\
\hline Pochonia chlamydosporia var. catenulata & CBS 504.66 & AF339593 & AF339544 & EF469069 & EF469098 & EF469120 \\
\hline Purpureocillium lilacinum & CBS 284.36 & AY526475 & FR775484 & EF468792 & EF468898 & EF468941 \\
\hline
\end{tabular}


Table 1 (continued)

\begin{tabular}{|c|c|c|c|c|c|c|}
\hline \multirow[t]{2}{*}{ Taxon } & \multirow[t]{2}{*}{ Voucher information } & \multicolumn{5}{|c|}{ GenBank accession number } \\
\hline & & $\mathrm{nrSSU}$ & $\operatorname{nr} L S U$ & tef- $1 \alpha$ & $r p b 1$ & $r p b 2$ \\
\hline Samsoniella alboaurantium & CBS 240.32 & JF415958 & JF415979 & JF416019 & JN049895 & JF415999 \\
\hline Samsoniella alboaurantium & CBS 262.58 & MH869308 & AB080087 & MF416497 & MF416654 & MF416448 \\
\hline Samsoniella alpina & YFCC 5818 & MN576753 & MN576809 & MN576979 & MN576869 & MN576923 \\
\hline Samsoniella alpina & YFCC 5831 & MN576754 & MN576810 & MN576980 & MN576870 & MN576924 \\
\hline Samsoniella alpina & YFCC 5836 & MN576755 & MN576811 & MN576981 & MN576871 & MN576925 \\
\hline Samsoniella antleroides & YFCC 6016 & MN576747 & MN576803 & MN576973 & MN576863 & MN576917 \\
\hline Samsoniella antleroides & YFCC 6113 & MN576748 & MN576804 & MN576974 & MN576864 & MN576918 \\
\hline Samsoniella aurantia & TBRC 7271 & & MF140728 & MF140846 & MF140791 & MF140818 \\
\hline Samsoniella aurantia & TBRC 7272 & & MF140727 & MF140845 & & MF140817 \\
\hline Samsoniella cardinalis & YFCC 5830 & MN576732 & MN576788 & MN576958 & MN576848 & MN576902 \\
\hline Samsoniella cardinalis & YFCC 6144 & MN576730 & MN576786 & MN576956 & MN576846 & MN576900 \\
\hline Samsoniella cardinalis & YFCC 6320 & MN576731 & MN576787 & MN576957 & MN576847 & MN576901 \\
\hline Samsoniella cristata & YFCC 6021 & MN576735 & MN576791 & MN576961 & MN576851 & MN576905 \\
\hline Samsoniella cristata & YFCC 6023 & MN576736 & MN576792 & MN576962 & MN576852 & MN576906 \\
\hline Samsoniella cristata & YFCC 7004 & MN576737 & MN576793 & MN576963 & MN576853 & MN576907 \\
\hline Samsoniella hepiali & ICMM 82-2 & MN576738 & MN576794 & MN576964 & MN576854 & MN576908 \\
\hline Samsoniella hepiali & Cor-4 & MN576743 & MN576799 & MN576969 & MN576859 & MN576913 \\
\hline Samsoniella hepiali & YFCC 661 & MN576739 & MN576795 & MN576965 & MN576855 & MN576909 \\
\hline Samsoniella hepiali & YFCC 2702 & MN576740 & MN576796 & MN576966 & MN576856 & MN576910 \\
\hline Samsoniella hepiali & YFCC 5823 & MN576745 & MN576801 & MN576971 & MN576861 & MN576915 \\
\hline Samsoniella hepiali & YFCC 5828 & MN576744 & MN576800 & MN576970 & MN576860 & MN576914 \\
\hline Samsoniella hepiali & YFCC 7024 & MN576741 & MN576797 & MN576967 & MN576857 & MN576911 \\
\hline Samsoniella hepiali & YFCC 7215 & MN576742 & MN576798 & MN576968 & MN576858 & MN576912 \\
\hline Samsoniella inthanonensis & TBRC 7915 & & MF140725 & MF140849 & MF140790 & MF140815 \\
\hline Samsoniella kunmingensis & YHH 16002 & MN576746 & MN576802 & MN576972 & MN576862 & MN576916 \\
\hline Samsoniella lanmaoa & YFCC 6148 & MN576733 & MN576789 & MN576959 & MN576849 & MN576903 \\
\hline Samsoniella lanmaoa & YFCC 6193 & MN576734 & MN576790 & MN576960 & MN576850 & MN576904 \\
\hline Samsoniella ramosa & YFCC 6020 & MN576749 & MN576805 & MN576975 & MN576865 & MN576919 \\
\hline Samsoniella tortricidae & YFCC 6013 & MN576751 & MN576807 & MN576977 & MN576867 & MN576921 \\
\hline Samsoniella tortricidae & YFCC 6131 & MN576750 & MN576806 & MN576976 & MN576866 & MN576920 \\
\hline Samsoniella tortricidae & YFCC 6142 & MN576752 & MN576808 & MN576978 & MN576868 & MN576922 \\
\hline Samsoniella yunnanensis & YFCC 1527 & MN576756 & MN576812 & MN576982 & MN576872 & MN576926 \\
\hline Samsoniella yunnanensis & YFCC 1824 & MN576757 & MN576813 & MN576983 & MN576873 & MN576927 \\
\hline Samsoniella yunnanensis & YFCC 7282 & MN576758 & MN576814 & MN576984 & MN576874 & MN576928 \\
\hline Shimizuomyces paradoxus & EFCC 6279 & EF469131 & EF469084 & EF469071 & EF469100 & EF469117 \\
\hline Simplicillium lamellicola & CBS 116.25 & AF339601 & AF339552 & DQ522356 & DQ522404 & DQ522462 \\
\hline Simplicillium lanosoniveum & CBS 704.86 & AF339602 & AF339553 & DQ522358 & DQ522406 & DQ522464 \\
\hline Simplicillium lanosoniveum & CBS 101267 & AF339603 & AF339554 & DQ522357 & DQ522405 & DQ522463 \\
\hline Simplicillium obclavatum & CBS 311.74 & AF339567 & AF339517 & EF468798 & & \\
\hline Simplicillium yunnanense & YFCC 7133 & MN576728 & MN576784 & MN576954 & MN576844 & \\
\hline Simplicillium yunnanense & YFCC 7134 & MN576729 & MN576785 & MN576955 & MN576845 & \\
\hline Sphaerostilbella berkeleyana & CBS 102308 & AF543770 & U00756 & AF543783 & AY489671 & DQ522465 \\
\hline Tolypocladium inflatum & OSC 71235 & EF469124 & EF469077 & EF469061 & EF469090 & EF469108 \\
\hline Tolypocladium japonicum & OSC 110991 & DQ522547 & DQ518761 & DQ522330 & DQ522375 & DQ522428 \\
\hline Torrubiella ratticaudata & ARSEF 1915 & DQ522562 & DQ518777 & DQ522360 & DQ522408 & DQ522467 \\
\hline Torrubiella sp. & DJ 29 & EU369108 & & EU369027 & EU369065 & \\
\hline Torrubiella sp. & NHJ 7859 & EU369107 & & & EU369064 & EU369085 \\
\hline Torrubiella wallacei & CBS 101237 & AY184978 & AY184967 & EF469073 & EF469102 & EF469119 \\
\hline Trichoderma deliquescens & ATCC 208838 & AF543768 & AF543791 & AF543781 & AY489662 & DQ522446 \\
\hline Trichoderma stercorarium & ATCC 62321 & AF543769 & AF543792 & AF543782 & AY489633 & EF469103 \\
\hline
\end{tabular}




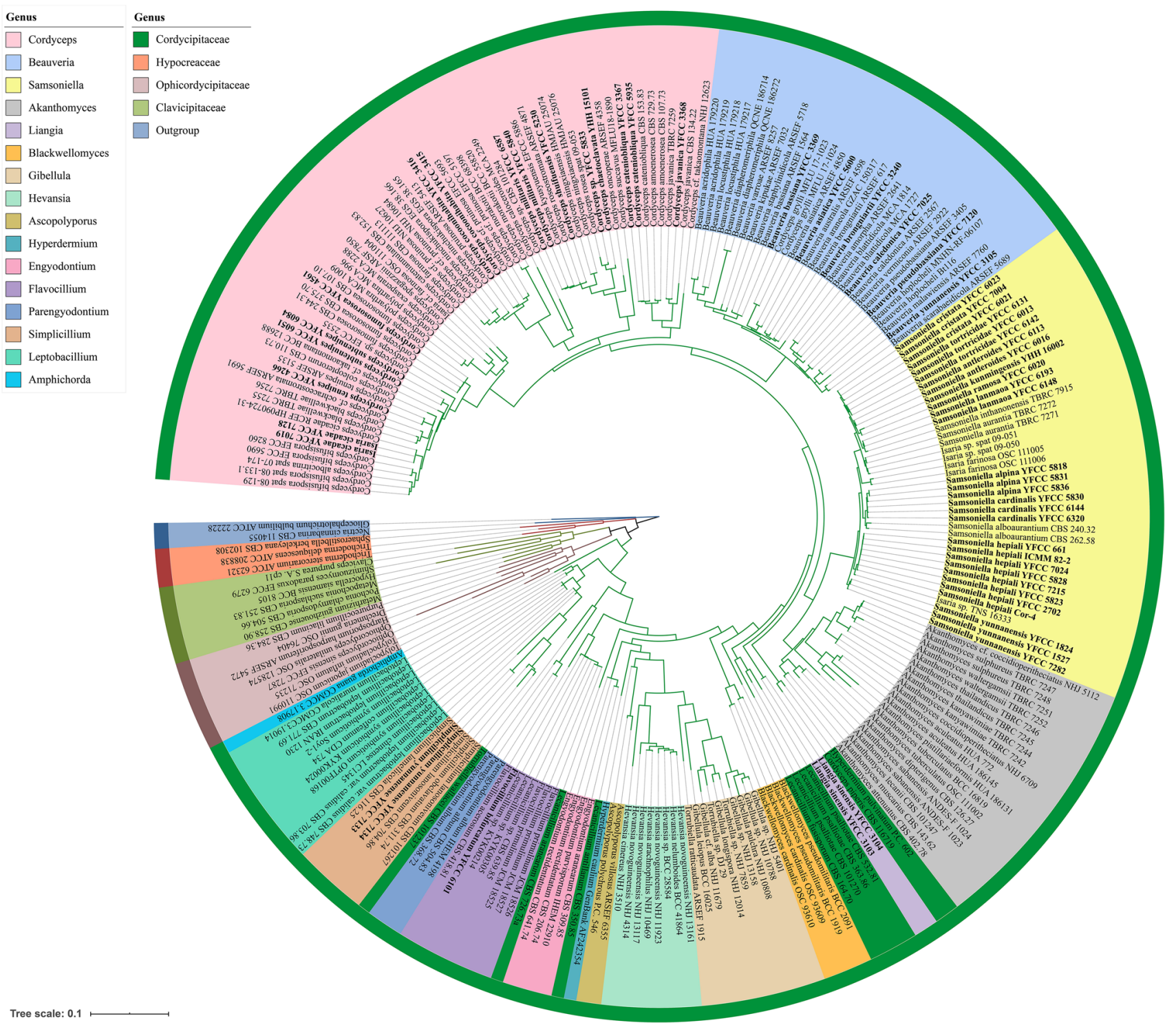

Fig. 1 Phylogenetic tree of Cordycipitaceae and related families inferred from a multigene dataset $(\mathrm{nr} L S U, \mathrm{nrSSU}, t e f-1 \alpha, r p b 1$ and $r p b 2$ ) based on maximum likelihood (ML) and Bayesian inference (BI) analyses. Families of Hypocreales and genera of Cordycipitaceae

analyses based on ITS sequences containing 655 bp from 30 taxa arranged in Lecanicillium and Simplicillium clarified further phylogenetic relationships in Lecanicillium. The ITS phylogenetic tree showed that the Lecanicillium lineage was polyphyletic and composed of eight monophyletic clades. In addition to $L$. primulinum, $L$. acerosum, Lecanicillium sp. and one new species (YHH 15428, YFCC 6101), the $L$. primulinum clade contained one recently described species $L$. subprimulinum S.K. Huang \& K.D. Hyde (Fig. 3). Five-gene phylogenetic analyses showed that a Lecanicillium-like species (YFCC 3103, YFCC 3104) isolated from B. yunnanensis Z.H. Chen \& L. Xu was clustered in the central portion of are differentiated with different colors. Phylogenetic relationships among families of Hypocreales are strongly supported by ML bootstrap proportions and $\mathrm{BI}$ posterior probabilities ( $\mathrm{BP} \geq 70 \%, \mathrm{PP} \geq 95 \%$ )

phylogenetic tree within Cordycipitaceae and formed a monophyletic clade, being well supported by analyses $(\mathrm{BP}=75 \%$, $\mathrm{PP}=100 \%$ ) (Fig. 1, 2).

The systematic position of $P$. hepiali was determined by five-gene phylogeny with the holotype living culture ICMM 82-2 and seven other samples. These eight samples closely clustered together with a well-supported clade and were placed in the genus Samsoniella, all of which were phylogenetically distinct from $C$. farinosa (Holmsk.) Kepler et al. (type strain CBS 111113) belonging to the type genus Cordyceps of Cordycipitaceae (Fig. 1). Nine undescribed species collected from Yunnan in China also clustered in the 


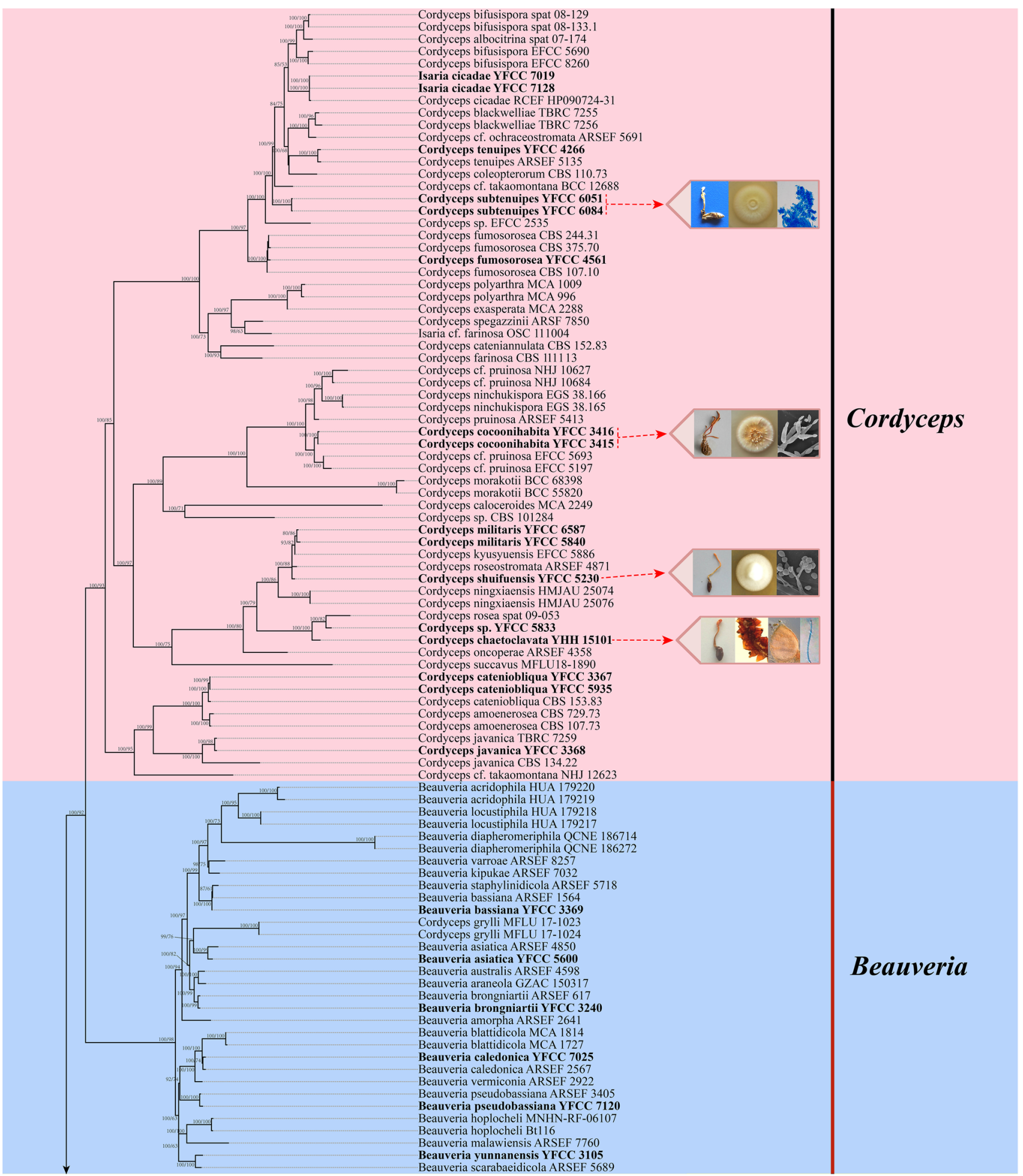

Fig. 2 Phylogenetic relationships among genera and related species in the family Cordycipitaceae inferred from a multigene dataset ( $\mathrm{nr} L S U$, $\mathrm{nrSSU}$, tef- $1 \alpha, r p b 1$ and $r p b 2$ ) based on ML and BI analyses. Values at the nodes before and after the backslash are BI posterior probabilities and ML bootstrap proportions, respectively. Support values greater than $50 \%$ are indicated at the nodes 


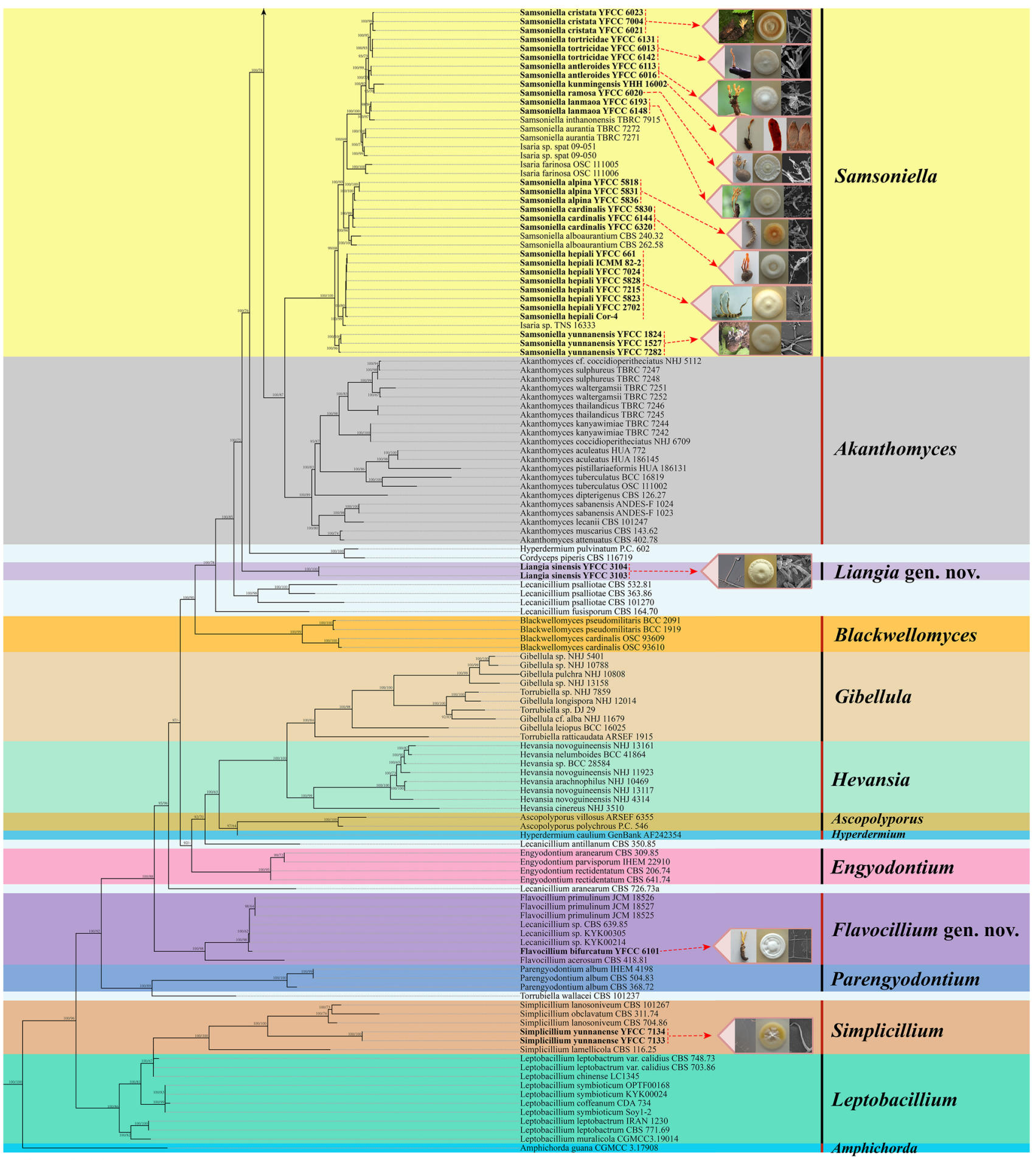

Tree scale: 0.1

Fig. 2 (continued)

genus Samsoniella and were clearly distinct from P. hepiali and three described species, viz. S. alboaurantia (G. Sm.) Mongkolsamrit et al., S. aurantia Mongkolsamrit et al. and S. inthanonensis Mongkolsamrit et al. (Fig. 1, 2). Five-gene phylogenetic analyses showed that four new species in Cordyceps and one new species in Simplicillium represented distinctive taxa in the family Cordycipitaceae. 


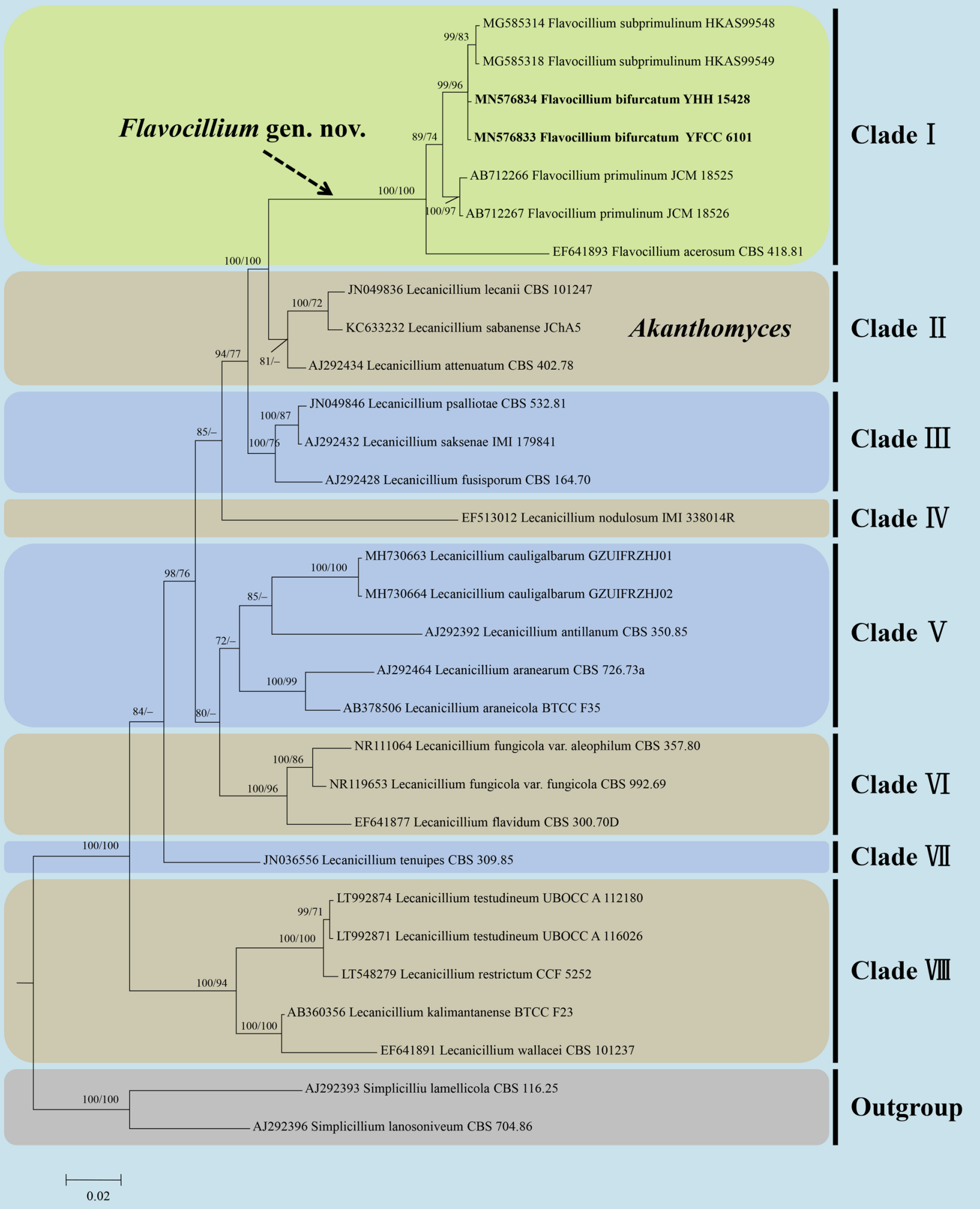

Fig. 3 Phylogenetic relationships of the genus Flavocillium and related Lecanicillium species in the family Cordycipitaceae inferred from ITS sequences based on ML and BI analyses. Statistical support values greater than $70 \%$ are shown at the nodes for BI posterior prob- abilities / ML boostrap proportions. Simplicillium lanosoniveum CBS 704.86 and Sim. lamellicola CBS 116.25 were designated as the outgroup taxa 

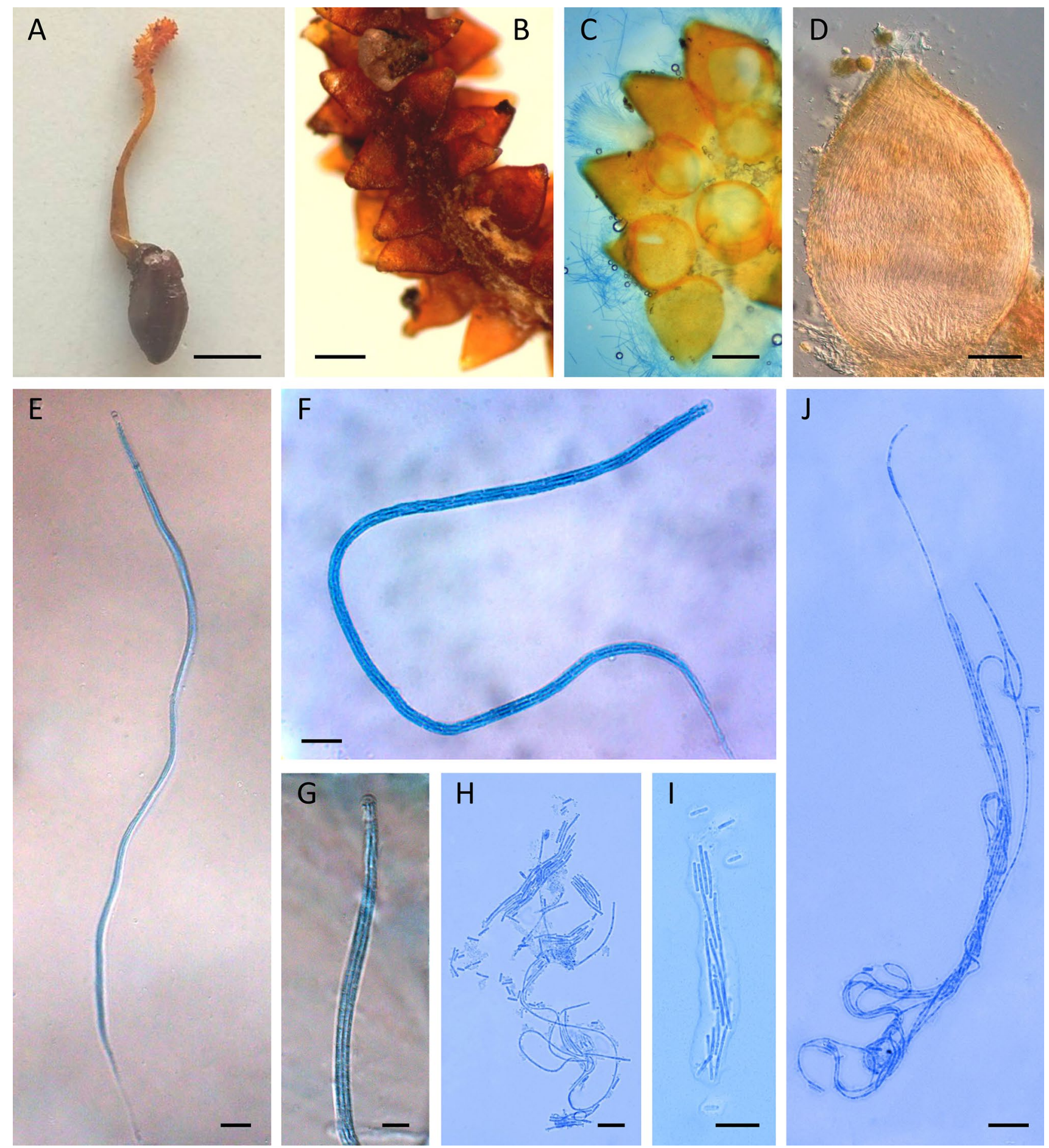

Fig. 4 Cordyceps chaetoclavata. A Fungus on the pupa of Lepidoptera. B Fertile part. C, D Perithecia. E-G Asci. H Ascospores and partspores. I Part-spores. J Ascospores. Scale bars: A =5 mm; B, C $=200 \mu \mathrm{m} ; \mathbf{D}=50 \mu \mathrm{m} ; \mathbf{E}, \mathbf{F}=10 \mu \mathrm{m} ; \mathbf{G}=5 \mu \mathrm{m} ; \mathbf{H}-\mathbf{J}=10 \mu \mathrm{m}$

\section{Taxonomy}

Cordyceps chaetoclavata H. Yu, Y.B. Wang, Y. Wang, Q. Fan \& Zhu L. Yang, sp. nov.

Mycobank: MB 833090; Fig. 4
Etymology: Referring to the clavate stromata with spinous fertile parts.

Holotype: YHH 15101.

Sexual morph: Stroma arising from the pupa of Lepidoptera buried in soil, solitary, cylindrical, reddish-orange 
to crimson, tapering gradually toward the apex, $2.3 \mathrm{~cm}$ long. Stipe cylindrical, orange to reddish-orange, $0.8 \mathrm{~mm}$ wide. Fertile part clavate, reddish-orange, covered by a spinous surface, up to $5.6 \mathrm{~mm}$ long, $0.7-1.1 \mathrm{~mm}$ wide, with sparsely distributed perithecia. Perithecia lageniform, crimson, superficial, 402-610 $\times 280-427 \mu \mathrm{m}$. Asci cylindrical, eightspored, 274-385 $\times 3.7-4.8 \mu \mathrm{m}$, with a hemispheric apical cap of 3.1-4.3×2.1-2.7 $\mu \mathrm{m}$. Ascospores 127-260×0.9-1.2 $\mu \mathrm{m}$, filiform, multiseptate, breaking into cylindrical part-spores of 3-12 $\mu \mathrm{m}$ long. Asexual morph: Undetermined.

Host: Pupa of Lepidoptera.

Habitat: On the pupa of Lepidoptera buried in soil.

Distribution: Kunming City, China.

Material examined: CHINA. YUNNAN PROVINCE: Kunming City, Wild Duck Lake Forest Park, on the pupa of Lepidoptera buried in soil, 12 August 2017, Qi Fan, (YHH 15101, holotype).

Notes: Five-gene phylogenetic analyses show that $C$. chaetoclavata is sister to $C$. rosea Kobayasi \& Shimizu (spat 09-053) and an undescribed Cordyceps species (YFCC 5833). Based on the original description of C. rosea, it has rose stromata (1.1 cm long), immersed perithecia with ovoid shape, and the host of lepidopteran larvae (Kobayasi and Shimizu 1982). However, C. chaetoclavata differs from $C$. rosea by its longer stromata $(2.3 \mathrm{~cm}$ long) with reddishorange to crimson colors, spinous fertile parts, superficial lageniform perithecia, and the host of lepidopteran pupae. In addition, $C$. chaetoclavata is also morphologically similar to those of $C$. militaris, $C$. ningxiaensis T. Bau \& J.Q. Yan, C. kyusyuensis Kawam, C. roseostromata Kobayasi \& Shimizu, C. shuifuensis and C. succavus Y.P. Xiao et al. by sharing fleshy and cylindrical stipes, and orange to reddishorange stromata (Kobayasi 1981; Kobayasi and Shimizu 1983; Liang 2007; Yang et al. 2012; Yan and Bau 2015; Hyde et al. 2019). The difference is that $C$. chaetoclavata has spinous fertile parts and superficial lageniform perithecia.

Cordyceps cocoonihabita H. Yu, Y.B. Wang, Y. Wang, Q. Fan \& Zhu L. Yang, sp. nov.

Mycobank: MB 833091; Fig. 5

Etymology: Referring to the host pupae of Lepidoptera inhabiting cocoons.

Holotype: YHH 8004.

Sexual morph: Stromata two or several, arising from the oval cocoon of the insect host, orange to pink, cylindrical, 15.2-57.8 $\mathrm{mm}$ long, unbranched or sometimes terminal branched. Stipes cylindrical, pink to reddishorange, $0.1-1.3 \mathrm{~mm}$ wide. Fertile parts clavate, orange to pink or reddish-orange, $3.5-17.4 \times 0.3-1.5 \mathrm{~mm}$, often with aperithecial apices. Perithecia superficial, oblong-ovate, 346-435 × 125-199 $\mu \mathrm{m}$. Asci cylindrical, 205-330 $\times 2.1-3.3 \mu \mathrm{m}$, eight-spored, with a hemispheric apical cap of 2.9-4.2 $\times 2.1-3.0 \mu \mathrm{m}$. Ascospores hyaline, cylindrical, septate, $140-269 \times 1.4-2.1 \mu \mathrm{m}$, disarticulating into cylindrical part-spores of 2.9-8.0 $\mu \mathrm{m}$ long. Asexual morph: Isaria-like. Colonies on PDA moderately fastgrowing, 38-45 mm diameter in 14 days at $25^{\circ} \mathrm{C}$, cottony, with high mycelial density; yellowish to pale orange at the centrum, white to yellowish at the edge, generating radially distributed stromata after 10 days, reverse brown. Hyphae smooth, septate, hyaline, 1.3-2.4 $\mu \mathrm{m}$ wide. Conidiophores smooth-walled, cylindrical, 5.8-8.3 $\times 1.4-2.0 \mu \mathrm{m}$. Phialides cylindrical to flask-shaped, solitary, alternate or whorled, 4.0-16.7 $\mu \mathrm{m}$ long, tapering gradually or abruptly from $1.5-2.7 \mu \mathrm{m}$ at the base to $0.5-1.2 \mu \mathrm{m}$ at the apex. Conidia in chains or solitary, hyaline, oval to fusiform, one-celled, 1.6-3.0 $\times 0.7-1.5 \mu \mathrm{m}$.

Host: Pupae of Limacodidae.

Habitat: On the pupae of Limacodidae in cocoons buried in soil.

Distribution: Kunming City, China.

Material examined: CHINA. YUNNAN PROVINCE: Kunming City, Shuanglong Village, on the pupa of Limacodidae in a cocoon buried in soil, 21 July 2011, YuanBing Wang, (YHH 8004, holotype; YFCC 3415, ex-holotype living culture); Ibid., (YHH 8005, paratype; YFCC 3416, ex-paratype living culture). Kunming City, Xishan Forest Park, on the pupa of Lepidoptera in a cocoon buried in soil, 13 August 2018, Yuan-Bing Wang, (YHH 16246; YFCC 6569, living culture).

Notes: Cordyceps cocoonihabita is characterized by unbranched or terminally branched stromata, clavate fertile parts often have aperithecial apices, orange to pink or reddish-orange, superficial perithecia with oblong-ovate shape, cylindrical ascospores, and the host of lepidopteran pupae in oval cocoons. The asexual morph from PDA culture produces conidiophores with cylindrical to flask-shaped phialides which are monothetic, alternate or whorled, as well as oval to fusiform conidia in chains.

It is phylogenetically closely related to a formally undescribed taxon $C$. cf. pruinosa (EFCC 5197, EFCC 5693) and is separated from C. pruinosa Petch and C. ninchukispora (C.H. Su \& H.H. Wang) G.H. Sung et al. in this clade. Cordyceps cocoonihabita, C. pruinosa and C. ninchukispora have the similar macromorphological characteristics of stromata with orange to pink colors, pyriformlike perithecia, with the exception of the former fertile parts often have aperithecial apices (Petch 1924; Su and Wang 1986). The former two taxa have similar hosts of lepidopteran pupae in cocoons, they differ, however, from C. ninchukispora with hosts such as seeds of Beilschmiedia Nees. Ecologically, C. cocoonihabita and C. obliquiordinata Kobayasi \& Shimizu have similar habitats that are in cocoons of Lepidoptera (Kobayasi and Shimizu 1982). However, C. obliquiordinata is morphologically different from $C$. cocoonihabita by having shorter stromata, brevis stipes, ovoid and irregular pars fertile parts, obliquely 

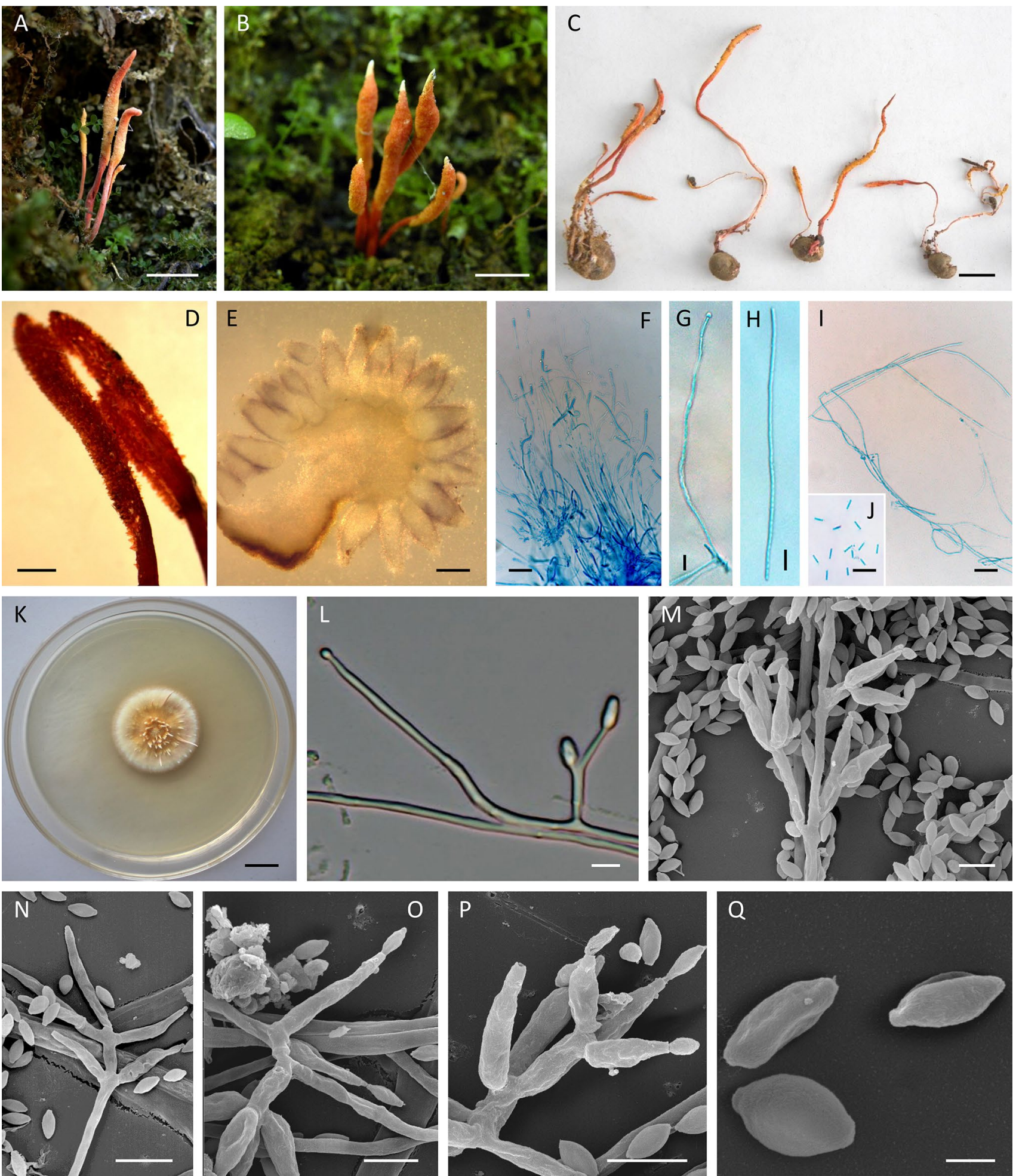

Fig. 5 Cordyceps cocoonihabita. A, B Stromata arising from hosts buried in soil. C Fungus on the pupae of Lepidoptera inhabiting cocoons. D Fertile part. E Perithecia. F, G Asci. H, I Ascospores. J Part-spores. K Colony on PDA. L-P Conidiophores and phialides. Q
Conidia. Scale bars: A-C $=1 \mathrm{~cm} ; \mathbf{D}=1 \mathrm{~mm} ; \mathbf{E}=200 \mu \mathrm{m} ; \mathbf{F}=20 \mu \mathrm{m}$; $\mathbf{G}=10 \mu \mathrm{m} ; \mathbf{H}=20 \mu \mathrm{m} ; \mathbf{I}, \mathbf{J}=10 \mu \mathrm{m} ; \mathbf{K}=1 \mathrm{~cm} ; \mathbf{L}=5 \mu \mathrm{m} ; \mathbf{M}=2 \mu \mathrm{m} ;$ $\mathbf{N}-\mathbf{P}=5 \mu \mathrm{m} ; \mathbf{Q}=1 \mu \mathrm{m}$ 
immersed perithecia, fairly short asci and ascospores. In terms of asexual morph, C. cocoonihabita has Isaria-like micromorphological characteristics and is significantly different from C. pruinosa and C. ninchukispora which respectively have morphs of Mariannaea G. Arnaud and Acremonium Link (Liang et al. 1983, 1991; Su and Wang 1986).

Cordyceps shuifuensis H. Yu, Y.B. Wang, Y. Wang \& Zhu L. Yang, sp. nov.

Mycobank: MB 833092; Fig. 6

Etymology: Named after the location Shuifu City where this species was collected.

Holotype: YHH 14101.

Sexual morph: Stromata solitary, cylindrical to slightly clavate, $2.5 \mathrm{~cm}$ long. Stipes cylindrical, yellowish to orange, $21 \times 1 \mathrm{~mm}$. Fertile parts clavate, yellowish, reddish-orange, $4 \times 1.5 \mathrm{~mm}$. Perithecia ovoid, reddish-orange, losely-packed, pseudoimmersed, 450-620 $\times 300-430 \mu \mathrm{m}$. Asci cylindrical, $275-510 \times 3.5-5.2 \mu \mathrm{m}$, with a hemispheric apical cap of 3.2-4.8 $\times 2.3-3.2 \mu \mathrm{m}$. Ascospores filiform, multiseptate, $180-410 \times 1.2-1.7 \mu \mathrm{m}$, breaking into cylindrical part-spores of 2.8-6.5 $\mu \mathrm{m}$ long. Asexual morph: Verticillium-like. Colonies on PDA fast-growing, $45-50 \mathrm{~mm}$ diameter in 14 days at $25{ }^{\circ} \mathrm{C}$, white, cottony, with protuberant mycelial density at the centrum, reverse yellowish. Hyphae smooth-walled, branched, septate, hyaline, 0.9-2.1 $\mu \mathrm{m}$ wide. Conidiophores smooth-walled, solitary, cylindrical, 5.5-9.2×1.6-2.7 $\mu \mathrm{m}$. Phialides cylindrical or subulate, solitary or in whorls of two to three, 4.7-20.0 $\mu \mathrm{m}$ long, tapering gradually toward the apex, 1.1-2.0 $\mu \mathrm{m}$ wide at the base, $0.4-2.1 \mu \mathrm{m}$ wide at the apex. Conidia have two types, hyaline, smooth-walled, onecelled, single or usually aggregate in subglobose to ellipsoidal heads at the apex of phialides. Macroconidia clavate to oblong-ovate, $5.1-11.8 \times 1.3-2.4 \mu \mathrm{m}$. Microconidia globose to ellipsoidal, $1.8-3.0 \times 1.6-2.5 \mu \mathrm{m}$.

Host: Pupa of Lepidoptera.

Habitat: On the pupa of Lepidoptera buried in soil.

Distribution: Shuifu City, China.

Material examined: CHINA. YUNNAN PROVINCE: Shuifu City, Tongluoba National Nature Reserve, on the pupa of Lepidoptera buried in soil, 07 September 2016, Yong-Dong Dai, (YHH 14101, holotype; YFCC 5230, exholotype living culture).

Notes: Cordyceps shuifuensis phylogenetically clusters with C. militaris, C. kyusyuensis Kawam and C. roseostromata Kobayasi \& Shimizu, but is distinguished from these three by forming a separate clade in this group. This species is morphologically closest to $C$. militaris having cylindrical to slightly clavate stromata with yellowish to reddish-orange colors, superficial perithecia and Verticillium-like asexual morph, but differs from the latter in size. Cordyceps shuifuensis only has Verticillium-like asexual morph, whereas C. militaris has both Verticillium- and Isaria-like asexual morphs (Yang et al. 2012). Cordyceps kyusyuensis differs from $C$. shuifuensis by having mutiple rhizoid stromata, the host larvae of Sphingidae and being very large in size (Kobayasi 1981; Liang 2007). Cordyceps roseostromata differs from C. shuifuensis by its mutiple and rhizoid stromata, rose color, and the host larvae of Coleoptera (Kobayasi and Shimizu 1983).

Cordyceps subtenuipes H. Yu, Y.B. Wang, Y. Wang, D.E. Duan \& Zhu L. Yang, sp. nov.

Mycobank: MB 833093; Fig. 7

Etymology: Referring to morphologically resembling Cordyceps tenuipes but phylogenetically distinct.

Holotype: YHH 15016.

Sexual morph: Undetermined. Asexual morph: Isaria-like. Synnemata arising from the pupae of Lepidoptera. Synnemata erect, solitary or two, flexuous, white, fleshy, up to $1.5 \mathrm{~cm}$ long, with terminal branches of $3-5 \times 1.5-2.0 \mathrm{~mm}$. Stipes cylindrical, $1 \mathrm{~mm}$ wide, producing a mass of conidia at the branches of synnemata, powdery and floccose. Conidiophores grouped together on th apex of synnemata, biverticillate with phialides in whorls of three to seven, $3.0-5.6 \times 1.8-3.2 \mu \mathrm{m}$. Phialides with a globose basal portion, 3.7-6.2 $\times 2.3-3.9 \mu \mathrm{m}$, tapering abruptly into a narrow neck of $0.5-1.0 \mu \mathrm{m}$ wide. Conidia usually single, one-celled, smooth-walled, hyaline, fusiform or oval, $2.2-3.2 \times 1.5-2.7 \mu \mathrm{m}$. Colonies on PDA moderately fastgrowing, $50-54 \mathrm{~mm}$ diameter in 14 days at $25^{\circ} \mathrm{C}$, white to yellowish, cottony, with low mycelial density, reverse deep yellow. Hyphae smooth-walled, branched, septate, hyaline, 1.3-2.7 $\mu \mathrm{m}$ wide. Conidiophores erect, arising from the aerial and prostrate hyphae, solitary or verticillate, with phialides in whorls of two to six, smooth-walled, cylindrical to ellipsoidal, 3.5-8.6 $\times 1.5-2.9 \mu \mathrm{m}$. Phialides solitary or verticillate, $5.3-42.5 \mu \mathrm{m}$ long, with a cylindrical or flaskshaped basal portion, tapering gradually or abruptly toward the apex, 1.6-3.4 $\mu \mathrm{m}$ wide at the base, and 0.5-1.1 $\mu \mathrm{m}$ wide at the apex. Conidia hyaline, one-celled, ellipsoidal or fusiform, $1.9-3.4 \times 1.7-2.5 \mu \mathrm{m}$, often in chains.

Host: Pupae of Lepidoptera.

Habitat: On the pupae of Lepidoptera buried in soil.

Distribution: Lanping County and Kunming City, China.

Material examined: CHINA. YUNNAN PROVINCE: Nujiang Lisu Autonomous Prefecture, Lanping County, Tongdian Town, on the pupa of Lepidoptera buried in soil, 05 August 2017, Yuan-Bing Wang, (YHH 15016, holotype; YFCC 6051, ex-holotype living culture). Kunming City, Xishan Forest Park, on the pupa of Lepidoptera buried in soil, 13 August 2018, Yuan-Bing Wang, (YHH 15002; YFCC 6084, living culture).

Notes: Phylogenetically, the new species $C$. subtenuipes forms a separate clade from the other species of Cordyceps with high credible support (100\%). Cordyceps subtenuipes is similar to C. tenuipes (Peck) Kepler et al. by its 

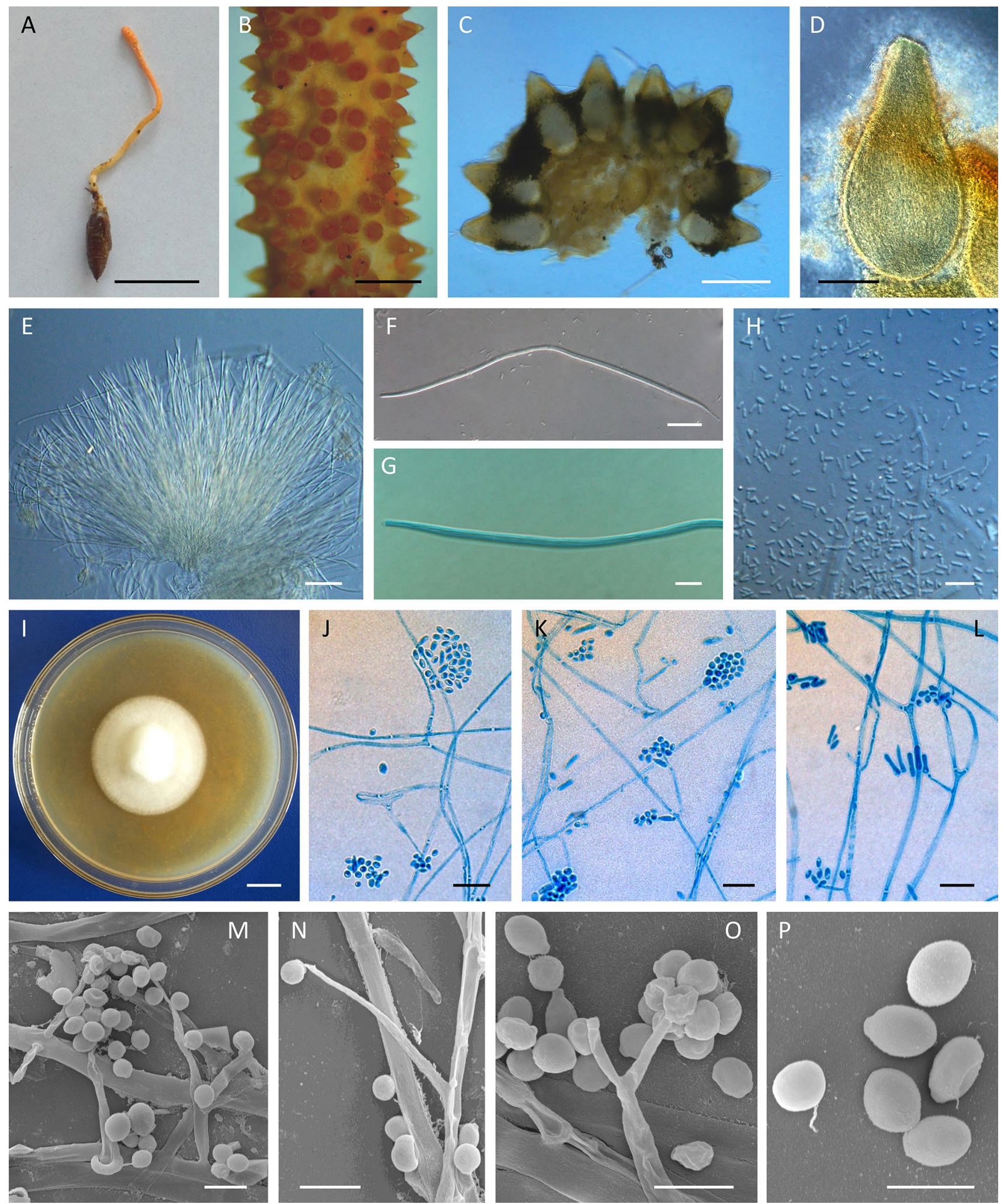

Fig. 6 Cordyceps shuifuensis. A Fungus on the pupa of Lepidoptera. B Fertile part. C, D Perithecia. E-G Asci. H Part-spores. I Colony on PDA. J-O Conidiophores and phialides. P Conidia. Scale bars:

$\mathbf{A}=1 \mathrm{~mm} ; \mathbf{B}, \mathbf{C}=500 \mu \mathrm{m} ; \mathbf{D}=200 \mu \mathrm{m} ; \mathbf{E}=50 \mu \mathrm{m} ; \mathbf{F}, \mathbf{G}=10 \mu \mathrm{m} ;$ $\mathbf{H}=5 \mu \mathrm{m} ; \mathbf{I}=1 \mathrm{~cm} ; \mathbf{J}-\mathbf{L}=10 \mu \mathrm{m} ; \mathbf{M}-\mathbf{O}=5 \mu \mathrm{m} ; \mathbf{P}=2 \mu \mathrm{m}$ 

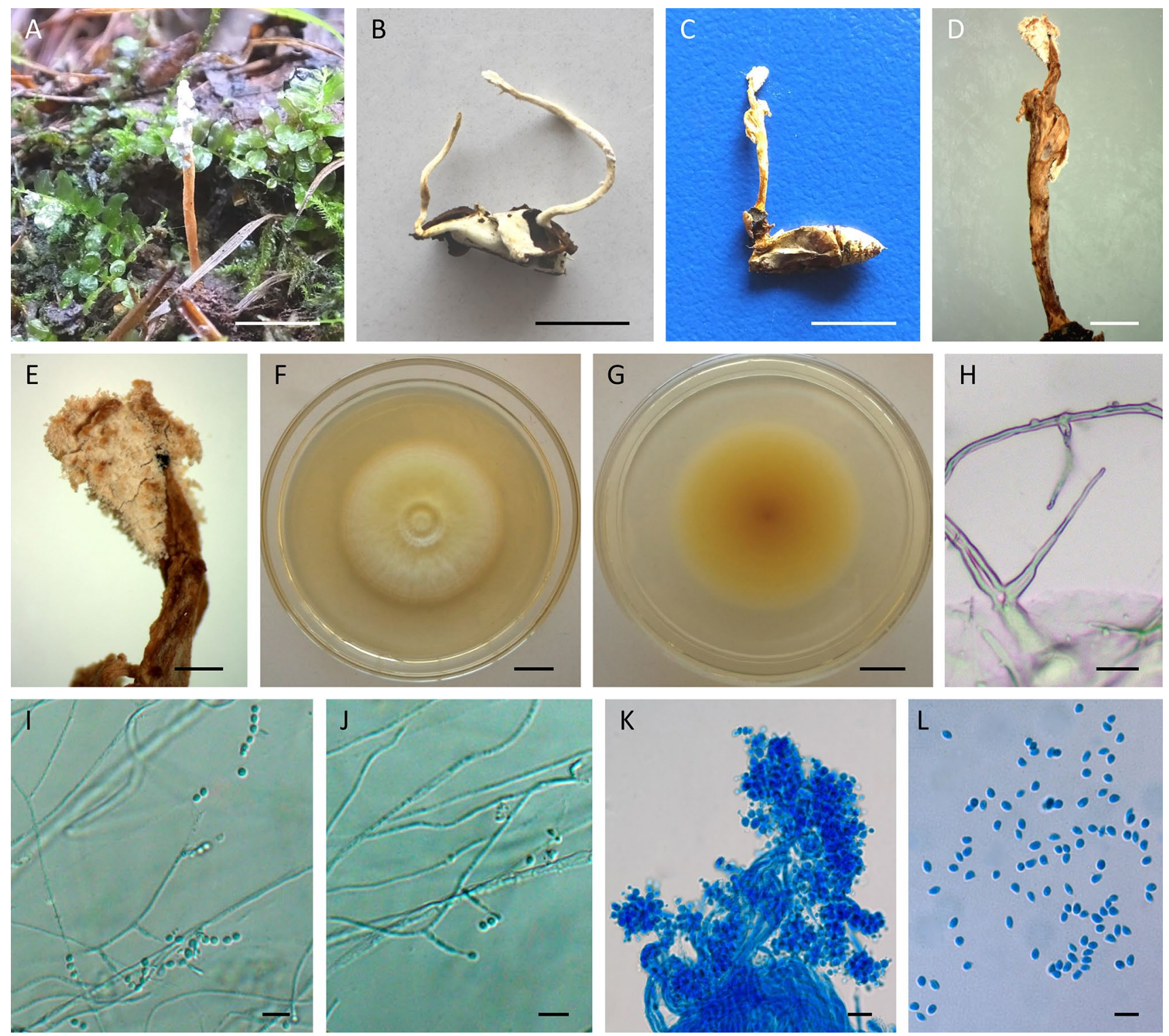

Fig. 7 Cordyceps subtenuipes. A Synnemata arising from the pupa of Lepidoptera buried in soil. B, C Fungus on the pupae of Lepidoptera. D, E Stipe producing a mass of conidia at the apex. F Colony

conspicuous synnemata and Isaria-like asexual conidiogenous structure producing phialides with a swollen basal portion. It differs from $C$. tenuipes by its single or two synnemata, white color, phialides with a globose basal portion and smaller fusiform or oval conidia measuring 1.9-3.4 × 1.5-2.7 $\mu \mathrm{m}$. Cordyceps tenuipes has mutiple synnemata, larger cylindrical to botuliform conidia with the size of $2.0-7.5 \times 1.0-2.5 \mu \mathrm{m}$ (Samson 1974). The sexual morph of $C$. tenuipes as proposed by the name $C$. takaomontana Yakush \& Kumaz has yellowish stromata and often co-occurs with its asexual morph (Liang 2007). However, the sexual morph of $C$. subtenuipes was not found in this study. on PDA. G Reverse of colony. H-K Conidiophores and phialides. L Conidia. Scale bars: $\mathbf{A}-\mathbf{C}=5 \mathrm{~mm} ; \mathbf{D}=1 \mathrm{~mm} ; \mathbf{E}=500 \mu \mathrm{m} ; \mathbf{F}$, $\mathbf{G}=1 \mathrm{~cm} ; \mathbf{H}=10 \mu \mathrm{m} ; \mathbf{I}, \mathbf{J}=5 \mu \mathrm{m} ; \mathbf{K}=10 \mu \mathrm{m} ; \mathbf{L}=5 \mu \mathrm{m}$

Flavocillium H. Yu, Y.B. Wang, Y. Wang, Q. Fan \& Zhu L. Yang, gen. nov.

Mycobank: MB 833094.

Etymology: Referring to the yellowish stromata and colonies.

Type species: Flavocillium bifurcatum H. Yu, Y.B. Wang, Y. Wang, Q. Fan \& Zhu L. Yang.

Sexual morph: Stromata arising from the insect buried in soil, clavate to flake-like, solitary, flexuous, yellowish, fleshy, up to $5 \mathrm{~cm}$ long, with a furcate terminal branch; stipe clavate, flexuous; fertile part contorted, clavate; perithecia densely packed, yellowish. Asexual morph: Lecanicilliumlike. Colonies yellowish, slow-growing. Conidiophores 
mononematous, cylindrical, with two to five phialides at the terminal nodes. Phialides lanceolate, solitary or in whorls of two to five, tapering gradually toward the apex. Two types of conidia hyaline, one-celled and smooth-walled, single or usually aggregate in subglobose to ellipsoidal heads at the apex of the phialides. Macroconidia fusiform, cymbiform or ellipsoidal to cylindrical. Microconidia oval to ellipsoidal or fusiform.

Notes: Five-gene phylogenetic analyses show that L. acerosum, L. primulinum, Lecanicillium sp. and our samples (YHH 15428, YFCC 6101) group together, in a monophyletic clade in the family Cordycipitaceae (Fig. 1, 2). This $L$. primulinum clade is clustered in the subbasal portion of phylogenetic tree within Cordycipitaceae and has a close phylogenetic relationship with Engyodontium and Parengyodontium, but forms a distinct lineage. ML and BI phylogenetic analyses based on ITS sequences from 30 taxa in Lecanicillium and Simplicillium show that the Lecanicillium group is polyphyletic and consists of eight monophyletic clades (Fig. 3). The L. primulinum clade includes L. acerosum, $L$. primulinum, Lecanicillium sp., L. subprimulinum and one new species with yellowish stromata (Fig. 3). This result is also supported by the previous phylogenetic analyses of Lecanicillium species from a combined $\mathrm{nrSSU}, \mathrm{nr} L S U$, tef-1 and ITS sequence dataset (Huang et al. 2018). In this clade, L. acerosum was first described by its distinguishing morphological characteristics producing the large straight macroconidia (Zare and Gams 2001). Recently, two species ( $L$. primulinum and L. subprimulinum) producing pastel yellow pigment were added, which were respectively isolated from soil and an ophioceras-like taxon on the dead submerged wood (Kaifuchi et al. 2013; Huang et al. 2018).

Morphologically, the L. primulinum clade is similar to other Lecanicillium species in terms of conidiophores, phialides and two types of conidia (Zare and Gams 2001; Zhou et al. 2018). However, these species of Flavocillium possess yellowish stromata with a furcate terminal branch, contorted fertile parts with yellowish perithecia and colonies that usually produce pastel yellow pigment, are obviously different from other members of the Lecanicillium lineage. In addition, the L. primulinum clade also can be distinguished from these phylogenetically related genera Engyodontium and Parengyodontium based on the morphological characteristics of the latters, both of which usually produce white colonies, conidiiferous rachids with denticles on phialides and terminal fertile regions that are zigzag-shaped (Gams et al. 1984; Tsang et al. 2016). Therefore, the new genus Flavocillium is introduced by the type species $F$. bifurcatum in order to accomodate the three following new combinations previously treated as members of Lecanicillium.

Flavocillium acerosum (W. Gams et al.) H. Yu, Y.B. Wang, Y. Wang \& Zhu L. Yang, comb. nov.

Mycobank: MB 833101.
Basionym: Lecanicillium acerosum W. Gams et al., Nova Hedwigia 73(1-2): 37 (2001).

Descriptions and illustrations: Zare and Gams (2001).

Distribution: Known from Brazil, Amazon (Zare and Gams 2001).

Notes: Flavocillium acerosum was first isolated from Crinipellis perniciosa (Stahel) Singer on Theobroma caocao Linn. (Zare and Gams 2001). This species is characterized by producing phialides solitary or up to two to five at the node, which gradually taper toward the apex (30-32 $\times 1.8-2.2 \mu \mathrm{m})$, fusiform macr- and microconidia with acute ends, presenting octahedral crystals (Zare and Gams 2001). It morphologically resembles L. antillanum, but its straight conidia are distinct from the sigmoidally curved conidia of the latter. Five-gene and ITS phylogenetic analyses indicate that $F$. acerosum and L. antillanum are located in different clades which represent a genus level difference in the family Cordycipitaceae.

Flavocillium bifurcatum H. Yu, Y.B. Wang, Y. Wang, Q. Fan \& Zhu L. Yang, sp. nov.

Mycobank: MB 833096; Fig. 8

Etymology: Referring to the stromata with a bifurcate terminal branch.

\section{Holotype: YHH 15428.}

Sexual morph: Stromata arising from the insect buried in soil, clavate to flake-like, solitary, flexuous, yellowish, fleshy, up to $2.0 \mathrm{~cm}$ long, with a furcate terminal branch of 13-16 × 1.5-2.0 mm. Stipe clavate, flexuous, $2.2 \mathrm{~mm}$ wide. Fertile parts contorted, clavate, 11-14×1.4-2.0 mm. Perithecia densely packed, yellowish, immature. Asci not observed. Asexual morph: Colonies on PDA slowgrowing, up to $3 \mathrm{~cm}$ diameter in 14 days at $25{ }^{\circ} \mathrm{C}$, white to yellowish, cottony with raised mycelial density at the centrum, generating several concentric rings at the edge, reverse pale yellow to brown. Hyphae hyaline, septate, branched, smooth-walled, 1.0-2.3 $\mu$ m wide. Conidiophores mononematous, cylindrical, 50.0-64.2 ×0.9-1.8 $\mu \mathrm{m}$. Phialides on conidiophores or arising from prostrate hyphae, lanceolate, solitary or in whorls of two to five, tapering gradually toward the apex, $18.1-44.5 \mu \mathrm{m}$ long, $1.1-2.4 \mu \mathrm{m}$ wide at the base, and 0.7-1.5 $\mu \mathrm{m}$ wide at the apex. Two types of conidia hyaline, one-celled and smooth-walled, single or usually aggregate in subglobose to ellipsoidal heads at the apex of the phialides. Macroconidia cymbiform, 5.5-9.2 × 1.3-2.7 $\mu \mathrm{m}$. Microconidia ellipsoidal to reniform, $2.1-4.2 \times 0.9-1.5 \mu \mathrm{m}$.

Host: Larva of Noctuidae.

Habitat: On the larva of Noctuidae buried in soil.

Distribution: Kunming City, China.

Material examined: CHINA. YUNNAN PROVINCE: Kunming City, Wild Duck Lake Forest Park, on the larva of Noctuidae buried in soil, 12 August 2017, Hong Yu, (YHH 15428, holotype; YFCC 6101, ex-holotype living culture). 

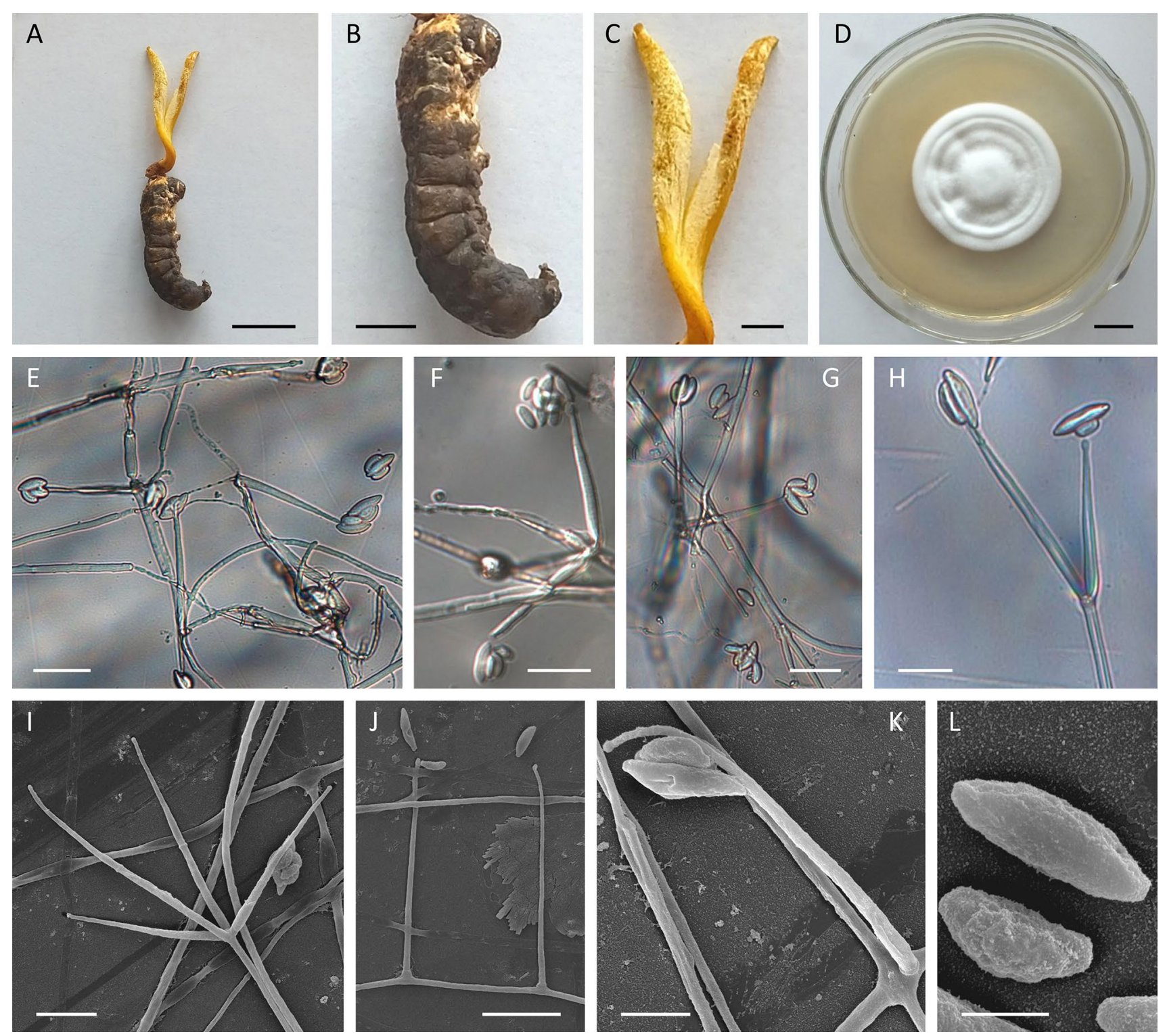

Fig. 8 Flavocillium bifurcatum. A Fungus on the host. B The host larva of Noctuidae. C Stroma with a bifurcate terminal branch. D Colony on PDA. E-I Conidiophores with phialides. J, K Phial-

Notes: Flavocillium bifurcatum is characterized by the fleshy stromata with a bifurcate terminal branch, solitary, yellowish, contorted fertile parts, long conidiophores, lanceolate phialides, two types of cymbiform macroconidia and ellipsoidal to reniform microconidia.

Five-gene phylogenetic analyses suggest that $F$. bifurcatum is close to Lecanicillium sp. and $F$. primulinum. In addition, ITS phylogenetic analyses from more complete sequence data in this clade show that $F$. bifurcatum is sister to $F$. subprimulinum. Morphologically, $F$. bifurcatum is similar to F. subprimulinum and F. primulinum by the yellowish colonies, solitary or whorled phialides, macro- and microconidia aggregate in subglobose to ellipsoidal heads ides with conidia. $\mathbf{L}$ Conidia. Scale bars: $\mathbf{A}=5 \mathrm{~mm} ; \mathbf{B}, \mathbf{C}=2 \mathrm{~mm}$; $\mathbf{D}=1 \mathrm{~cm} ; \mathbf{E}-\mathbf{J}=10 \mu \mathrm{m} ; \mathbf{K}=5 \mu \mathrm{m} ; \mathbf{L}=2 \mu \mathrm{m}$

at the apex of phialides (Kaifuchi et al. 2013; Huang et al. 2018). However, the sexual morphs of $F$. subprimulinum and $F$. primulinum have not been observed. Flavocillium bifurcatum differs from $F$. subprimulinum and $F$. primulinum by its cymbiform macroconidia and longer conidiophores up to $64 \mu \mathrm{m}$. Ecologically, F. bifurcatum is parasitic on the larva of Noctuidae buried in soil and is quite different from other congeneric species.

Flavocillium primulinum (Kaifuchi et al.) H. Yu, Y.B. Wang, Y. Wang \& Zhu L. Yang, comb. nov.

Mycobank: MB 833103.

Basionym: Lecanicillium primulinum Kaifuchi et al., Mycoscience. 54: 294-293 (2013). 
Descriptions and illustrations: Kaifuchi et al. (2013).

Distribution: Known from Okinawa Prefecture, Japan (Kaifuchi et al. 2013).

Notes: The type strain of $F$. primulinum was isolated from soil under an unidentified plant. It is characterized by phialides produced on prostrate aerial hyphae, solitary or in whorls of two to five which taper toward the apex, ellipsoidal to cylindrical macroconidia and oval to ellipsoidal microconidia aggregate in subglobose to ellipsoidal heads at the apex of the phialides, presenting octahedral crystals (Kaifuchi et al. 2013). Phylogenetically, this species is close to $F$. bifurcatum and $F$. subprimulinum, but it differs morphologically from $F$. bifurcatum by the latter's cymbiform macroconidia and smaller microconidia of $2.1-4.2 \times 0.9-1.5 \mu \mathrm{m}$ in size.

Flavocillium subprimulinum (S.K. Huang \& K.D. Hyde) H. Yu, Y.B. Wang, Y. Wang \& Zhu L. Yang, comb. nov. Mycobank: MB 833106.

Basionym: Lecanicillium subprimulinum S.K. Huang \& K.D. Hyde, Phytotaxa 348 (2): 102 (2018).

Descriptions and illustrations: Huang et al. (2018).

Distribution: Known from Baoshan City, China (Huang et al. 2018).

Notes: Flavocillium subprimulinum is characterized by solitary or two to three phialides on conidiophores arising from hyaline hyphae, with gregarious, ovoid to ellipsoidal conidia (Huang et al. 2018). Ecologically, this species is associated with a sexual morph of an ophioceras-like taxon on submerged wood and is different from those of $F$. bifurcatum on the larva of Noctuidae and $F$. subprimulinum isolated from soil. Phylogenetically, F. subprimulinum is sister to $F$. bifurcatum based on ITS phylogenetic analyses of Lecanicillium lineage, but it differs morphologically from $F$. bifurcatum because the latter has bifurcate stromata, cymbiform macroconidia and longer conidiophores.

Liangia H. Yu, Y.B. Wang, Y. Wang, Z.H. Chen \& Zhu L. Yang, gen. nov.

Mycobank: MB 833107.

Etymology: In honor of Prof. Zong-Qi Liang, acknowledging his contributions to our knowledge of cordycipitoid fungi.

Type species: Liangia sinensis H. Yu, Y.B. Wang, Y. Wang, Z.H. Chen \& Zhu L. Yang.

Colonies on PDA slow-growing, effuse or stellate, white, usually raising dome-shaped mycelial density with a sunken zone at the centrum, verrucose around the margin. Conidiophores not observed. Phialides lanceolate, occurring directly from the prostrate hyphae, solitary, gradually attenuated toward the apex. Two types of macro- and microconidia, aseptate, smooth-walled, one-celled, both of them existing singly or in pairs at the the apex of phialides. Macroconidia positioned at a right angle to the apex of phialides, straight, oblong-oval to fusiform. Microconidia oval to ellipsoidal.
Notes: Liangia sinensis, isolated from an entomopathogenic fungus B. yunnanensis, represents a well-supported monophyletic lineage in the family Cordycipitaceae (Fig. 1). The new genus Liangia with Lecanicillium-like asexual morph is proposed for the type species Lia. sinensis based on its phylogenetic placement. In this study, it appears more closely related to $C$. piperis (J.F. Bisch. \& J.F. White) D. Johnson et al. and L. psalliotae clades by the five-gene phylogenetic analyses. The genus Liangia is morphologically similar to these two clades which possess asexual morph of Lecanicillium (Zare and Gams 2001; Bischoff and White 2004). However, it differs from the latter two groups by the shape and size of its colonies, phialides and conidia.

Liangia sinensis H. Yu, Y.B. Wang, Y. Wang, Z.H. Chen \& Zhu L. Yang, sp. nov.

Mycobank: MB 833109; Fig. 9

Etymology: Named after China where the species is distributed.

Holotype: YHH 7455.

Sexual morph: Undetermined. Asexual morph: Lecanicillium-like. Strains isolated from the stromata of Beauveria yunnanensis associated with the pupa of Lepidoptera. Colonies on PDA slow-growing, 28-34 $\mathrm{mm}$ in diameter after 14 days at $25^{\circ} \mathrm{C}$, effuse or stellate, white, usually raising dome-shaped mycelial density with a sunken zone at the centrum, verrucose around the margin. Reverse pale brown, causing a brown concentric ring outside of the inoculum. Hyphae hyaline, septate, branched, smooth-walled, and 0.7-2.4 $\mu \mathrm{m}$ wide. Phialides lanceolate, occurring directly from the prostrate hyphae, solitary, gradually attenuated toward the apex, 16.7-59.0 $\mu \mathrm{m}$ long, 0.7-1.6 $\mu \mathrm{m}$ wide at the base and $0.3-0.7 \mu \mathrm{m}$ wide at the apex. Conidia existing in two types, macro- and microconidia, aseptate, hyaline, smooth-walled, one-celled, straight, both existing singly or in pairs at the apex of phialides. Macroconidia positioned at a right angle to the apex of phialides, oblong-oval to fusiform, 4.5-9.3 $\times 1.2-1.9 \mu \mathrm{m}$. Microconidia oval to ellipsoidal, $1.8-3.3 \times 1.1-1.8 \mu \mathrm{m}$.

Substratum: Beauveria yunnanensis.

Habitat: On the stromata of $B$. yunnanensis associated with the pupa of Lepidoptera buried in soil.

Distribution: Baoshan City, China.

Material examined: CHINA. YUNNAN PROVINCE: Baoshan City, Mangkuan Village, isolated from B. yunnanensis associated with the pupa of Lepidoptera buried in soil, 22 July 2016, Zi-Hong Chen, (YHH 7455, holotype; YFCC 3103, ex-holotype living culture); Ibid., (YHH 7456; YFCC 3104, living culture).

Notes: Liangia sinensis possesses Lecanicillium-like asexual morph and is characterized by white colonies forming a sunken zone at the centrum of dome-shaped mycelial density and verrucose around the margin, solitary and lanceolate phialides occurring directly from the prostrate 

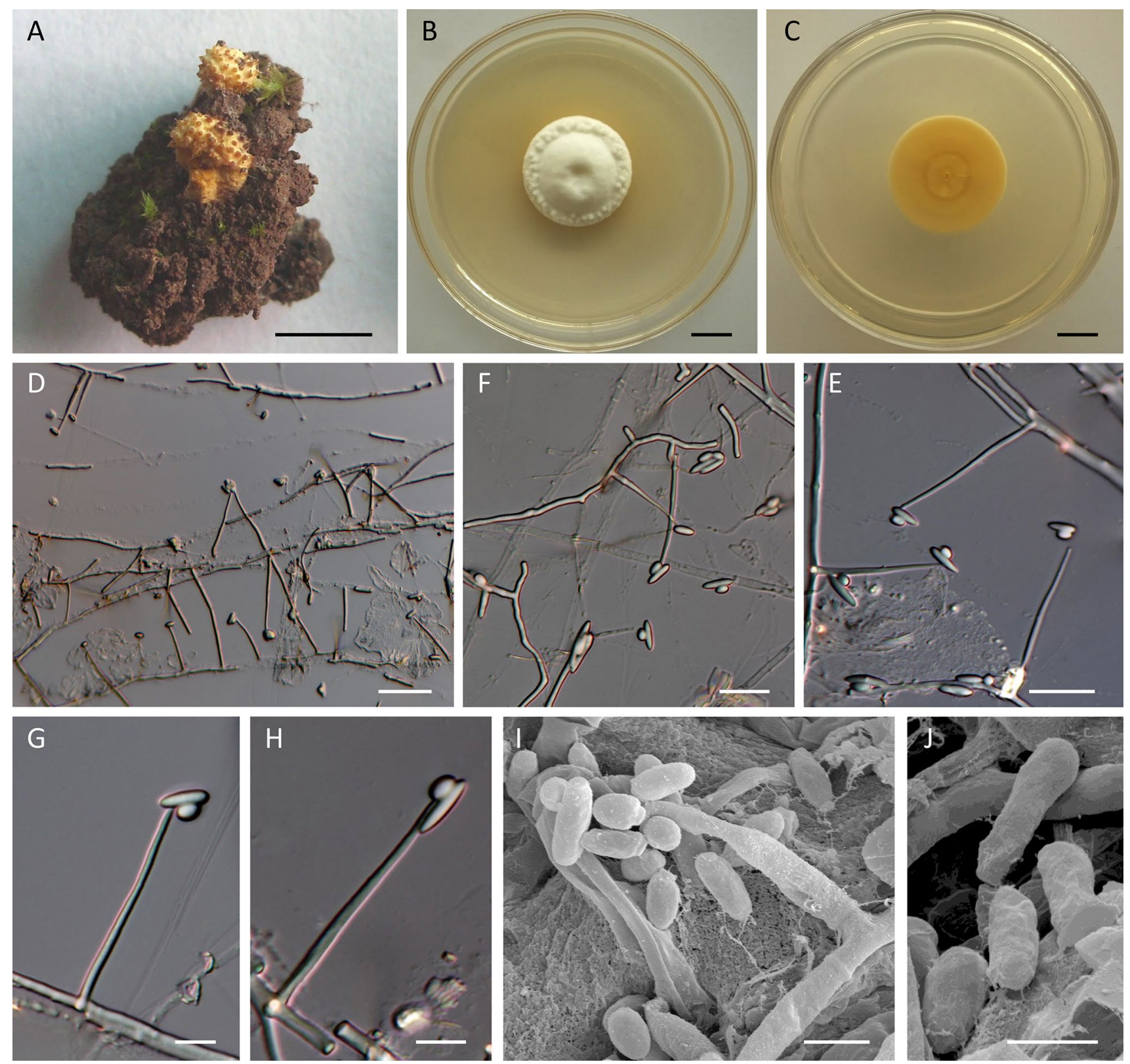

Fig. 9 Liangia sinensis. A Stromata of Beauveria yunnanensis arising from the pupa of Lepidoptera buried in soil, from which Lia. sinensis was isolated. B Colony on PDA. C Reverse of colony. D, E Phialides.

hyphae, oblong-oval to fusiform macroconidia, and oval to ellipsoidal microconidia existing singly or in pairs at the apex of phialides.

It is similar to the two phylogenetically more closely related $C$. piperis and $L$. psalliotae clades with asexual morph of Lecanicillium (Zare and Gams 2001; Bischoff and White 2004). However, Lia. sinensis differs from $C$. piperis and L. psalliotae by its distinguished colonies, solitary and lanceolate phialides without conidiophores and oblong-oval to fusiform macroconidia. Cordyceps piperis,
F-H Macro- and microconidia existing in pairs at the apex of phialides. I Phialides and conidia. J Conidia. Scale bars: $\mathbf{A}=5 \mathrm{~mm} ; \mathbf{B}$, $\mathbf{C}=1 \mathrm{~cm} ; \mathbf{D}=20 \mu \mathrm{m} ; \mathbf{E}, \mathbf{F}=10 \mu \mathrm{m} ; \mathbf{G}-\mathbf{I}=5 \mu \mathrm{m} ; \mathbf{J}=2 \mu \mathrm{m}$

originally named $T$. piperis J.F. Bischoff \& J.F. White, was reported to have the sexual morph of Torrubiella with sessile perithecial stromata which covered the corpses of scale insects attached to Piperaceae (Bischoff and White 2004). Lecanicillium psalliotae was originally described as Verticillium psalliotae Treschew which caused diseases of cultivated mushrooms (Treschew 1941), and later were widely discovered from insects, nematodes, soil, mushrooms, Rhopalomyces Corda and other fungi (Dayal and Barron 1970; Zare and Gams 2001; Yang et al. 2005). Liangia sinensis is 
distinctive for its isolates from the newly described cordycipitoid fungus $B$. yunnanensis parasitic on the lepidopteran pupa (Chen et al. 2019).

In the five-gene phylogenetic tree, B. yunnanensis (exholotype living culture CCTCC AF $2018010=$ YFCC 3105 ) is closely clustered with B. scarabaeidicola (Kobayasi) S.A. Rehner \& Kepler, and remotely related to Lia. sinensis (Fig. 1, 2). In this study, there is no strong hyperparasitic evidence that Lia. sinensis grows on the stromata of B. yunnanensis. However, two strains of Lia. sinensis were truly isolated from the stromata of $B$. yunnanensis. The possibility that Lia. sinensis is a hyperparasitic fungus of $B$. yunnanensis requires confirmation.

Samsoniella alpina H. Yu, Y.B. Wang, Y. Wang \& Zhu L. Yang, sp. nov.

Mycobank: MB 833110; Fig. 10
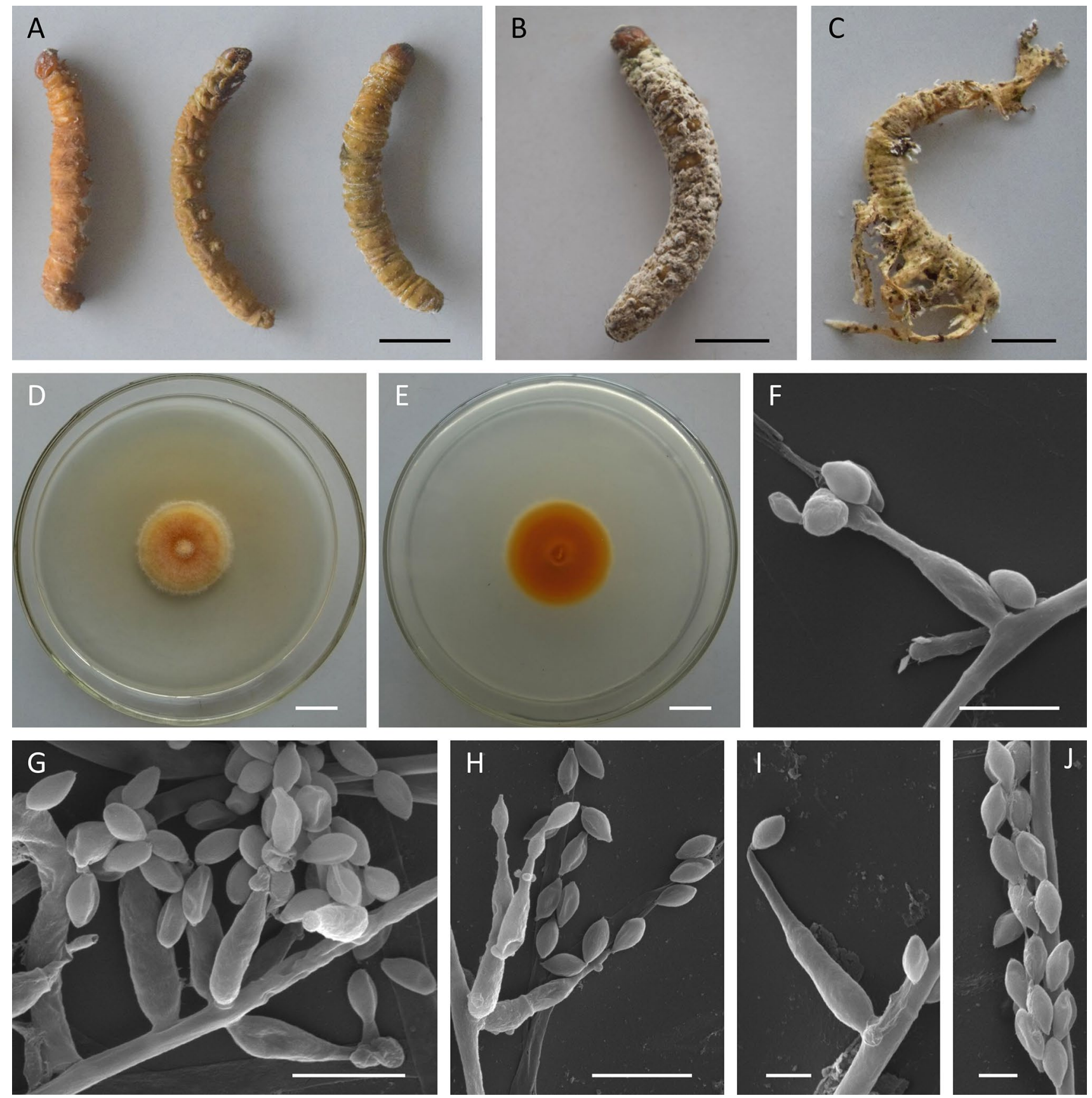

Fig. 10 Samsoniella alpina. A, B Larvae of Hepialus baimaensis infected by S. alpina. C Synnemata arising from the whole body of H. baimaensis. D Colony on PDA. E Reverse of colony. F Solitary phialides on hyphae. G, H Verticillate phialides. I Solitary phialides on hyphae. J Conidia in chains. Scale bars: $\mathbf{A}-\mathbf{E}=1 \mathrm{~cm} ; \mathbf{F}-\mathbf{H}=5 \mu \mathrm{m}$; $\mathbf{I}, \mathbf{J}=2 \mu \mathrm{m}$ 
Etymology: Named after the alpine locations where this species is distributed.

\section{Holotype: YHH 15316}

Sexual morph: Undetermined. Asexual morph: Isarialike. Synnemata arising from the whole body of larvae of Hepialus. Synnemata irregularly branched, $0.3-2.0 \mathrm{~cm}$ long, $0.1-0.3 \mathrm{~mm}$ wide; cylindrical or clavate stipes with white powdery heads, white to orange yellow. Conidiophores on the apex of synnemata, biverticillate with phialides in whorls of two to seven, cylindrical, 4.5-7.2 $\times 1.5-3.0 \mu \mathrm{m}$. Phialides with a basal portion cylindrical to narrowly lageniform, 4.3-10.3 $\times 1.6-2.9 \mu \mathrm{m}$, tapering abruptly into a narrow neck of $0.5-1.0 \mu \mathrm{m}$ wide. Conidia in chains, one-celled, smoothwalled, hyaline, fusiform or oval, $2.2-2.9 \times 1.3-2.0 \mu \mathrm{m}$. Colonies on PDA growing fairly well at $25^{\circ} \mathrm{C}$, up to $40 \mathrm{~mm}$ diameter in 14 days, hairy, floccose, light orange to orangered. Reverse cream, turning yellowish. Hyphae smoothwalled, branched, septate, hyaline, 0.9-2.7 $\mu \mathrm{m}$ wide. Conidiophores cylindrical, usually biverticillate with phialides in whorls of two to seven, 3.1-6.5 $\times 1.6-2.8 \mu \mathrm{m}$. Phialides verticillate on conidiophores, solitary or verticillate on hyphae, occasionally verruculose, basal portion cylindrical to narrowly lageniform, tapering abruptly toward the apex, 4.7-9.5 $\mu \mathrm{m}$ long, 1.9-3.1 $\mu \mathrm{m}$ wide at the base, $0.5-1.1 \mu \mathrm{m}$ wide at the apex. Conidia one-celled, smooth-walled, hyaline, fusiform or oval, $2.0-3.1 \times 1.3-2.1 \mu \mathrm{m}$, often in chains.

Host: Larvae of Hepialus baimaensis Liang.

Habitat: On the larvae of Hepialus baimaensis (Hepialidae) buried in soil.

Distribution: Diqing Tibetan Autonomous Prefecture, China.

Material examined: CHINA. YUNNAN PROVINCE: Diqing Tibetan Autonomous Prefecture, Shangrila City, Xiaozhongdian Town, on the larva of Hepialus baimaensis (Hepialidae) buried in soil, 6 May 2017, Can-Ming Zhang, (YHH 15316, holotype; YFCC 5818, ex-holotype living culture); Ibid., (YHH 15317, paratype; YFCC 5831, ex-paratype living culture); Ibid., (YHH 15319, paratype; YFCC 5836, ex-paratype living culture).

Notes: Samsoniella alpina has Isaria-like asexual morph and is characterized by irregularly branched synnemata, cylindrical or clavate stipes with white powdery heads, white to orange yellow, hairy and floccose colonies with light orange to orange-red colors, solitary or verticillate phialides with cylindrical to narrowly lageniform basal portion, fusiform or oval conidia.

Samsoniella alpina is phylogenetically sister to $S$. cardinalis with high statistical supports by $\mathrm{BP}=99 \%$ and $\mathrm{PP}=100 \%$. It is similar to $S$. cardinalis in producing phialides with cylindrical to narrowly lageniform basal portion, fusiform or oval conidia. However, it differs from $S$. cardinalis by irregularly branched synnemata with white powdery heads, white to orange yellow, colonies produing light orange to orange-red colors and parasitizing larvae of H. baimaensis.

Samsoniella antleroides H. Yu, Y.B. Wang, Y. Wang, Q. Fan \& Zhu L. Yang, sp. nov.

Mycobank: MB 833111; Fig. 11

Etymology: Referring to the antler-like stromata.

Holotype: YHH 15758

Sexual morph: Stromata fasciculate, antler-like, arising from the larvae of Noctuidae, 22.3-57.8 mm long, cylindrical to clavate, with oblate terminal branches of 4.6-26.2 mm long. Stipes flexuous, $16.4-43.5 \times 0.7-2.2 \mathrm{~mm}$. Fertile parts clavate to flake-like, lateral sides usually have a longitudinal ditch without producing perithecia, orange to orangered, 6.3-9.5 $\times 0.6-2.3 \mathrm{~mm}$. Perithecia superficial, fusiform, 294-442 ×131-216 $\mu \mathrm{m}$. Asci eight-spored, hyaline, cylindrical, $160-248 \times 2.1-2.7 \mu \mathrm{m}$. Ascus caps hemispherical, 1.9-3.2 $\times 1.8-2.5 \mu \mathrm{m}$. Ascospores hyaline, bola-shaped, septate, $110-184 \times 0.8-1.3 \mu \mathrm{m}$, central part filiform, terminal part narrowly fusiform, don't disarticulate into partspores. Asexual morph: Isaria-like. Colonies fast-growing on PDA, 35-40 mm diameter in 14 days at $25^{\circ} \mathrm{C}$, white to light orange, cottony, producing high mycelial density at the centrum. Reverse light orange, turning deep yellow brown, appearing a brown concentric ring and radiate stria out of the inoculum. Hyphae smooth, septate, hyaline, 1.1-1.9 $\mu \mathrm{m}$ wide. Conidiophores cylindrical, solitary or verticillate, 3.5-9.7 $\times 1.3-3.2 \mu \mathrm{m}$. Phialides verticillate, in whorls of two to nine, sometimes solitary on hyphae, basal portion cylindrical to narrowly lageniform, tapering abruptly toward the apex; 3.5-16.3 $\mu \mathrm{m}$ long, $1.7-2.9 \mu \mathrm{m}$ wide at the base, and $0.5-1.0 \mu \mathrm{m}$ wide at the apex. Conidia one-celled, smoothwalled, hyaline, fusiform or oval, $2.3-3.5 \times 1.6-2.5 \mu \mathrm{m}$, often in chains.

Host: Larvae of Noctuidae.

Habitat: On the larvae of Noctuidae buried in soil.

Distribution: Kunming City, China.

Material examined: CHINA. YUNNAN PROVINCE: Kunming City, Wild Duck Lake Forest Park, on the larva of Noctuidae buried in soil, 12 August 2017, Hong Yu, (YHH 15758, holotype; YFCC 6016, ex-holotype living culture); Ibid., (YHH 16034, paratype; YFCC 6113, ex-paratype living culture).

Notes: Samsoniella antleroides is characterized by fasciculate and antler-like stromata with oblate terminal branches, clavate to flake-like fertile parts, orange to orangered, superficial and fusiform perithecia, cylindrical asci with bola-shaped ascospores, light orange to orange-red colonies, having Isaria-like asexual conidiogenous structure, and on the larvae of Noctuidae buried in soil.

Phylogenetic analyses reveal that $S$. antleroides forms a sister lineage with $S$. tortricidae and S. cristata. Samsoniella antleroides resembles the latter two species in having stromata with terminal branches, superficial and fusiform 

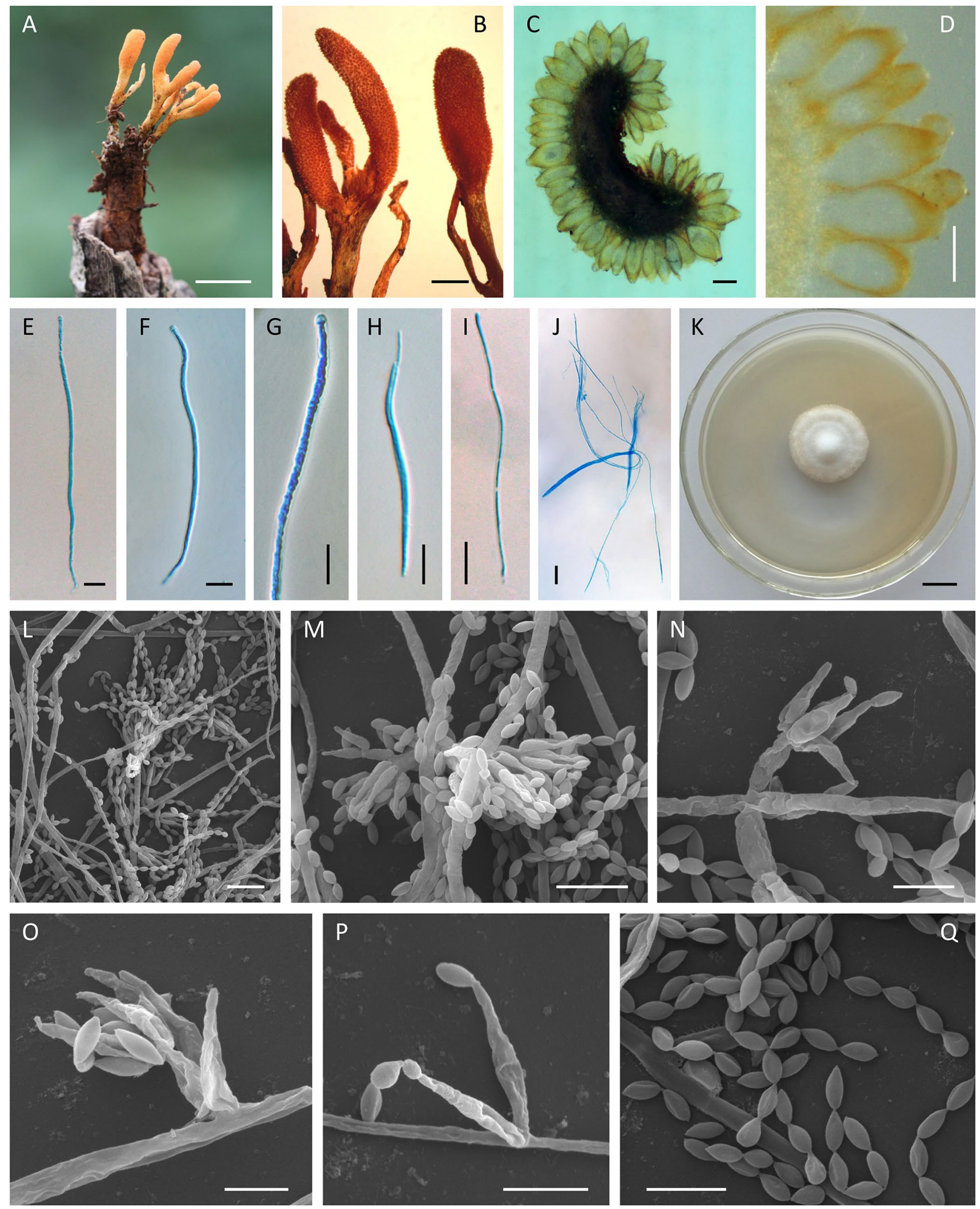

Fig. 11 Samsoniella antleroides. A Antler-like stromata arising from the larva of Noctuidae. B Fertile parts. C, D Perithecia. E-G Asci. H, I Immature ascospores. J Mature ascospores. K Colony on PDA. L-O Verticillate phialides on conidiophores. P Phialides in whorls of two on hypha. $\mathbf{Q}$ Conidia in chains. Scale bars: $\mathbf{A}=1 \mathrm{~cm}$; $\mathbf{B}=1 \mathrm{~mm} ; \mathbf{C}, \mathbf{D}=200 \mu \mathrm{m} ; \mathbf{E}, \mathbf{F}=20 \mu \mathrm{m} ; \mathbf{G}-\mathbf{J}=10 \mu \mathrm{m} ; \mathbf{K}=1 \mathrm{~cm} ; \mathbf{L}$, $\mathbf{M}=10 \mu \mathrm{m} ; \mathbf{N}-\mathbf{Q}=5 \mu \mathrm{m}$ 
perithecia, cylindrical asci with bola-shaped ascospores and Isaria-like asexual morph. However, it differs from S. cristata and S. tortricidae in the production of fasciculate and antler-like stromata with oblate terminal branches, clavate to flake-like fertile parts, conidiophores forming verticillate branches with shorter phialides in whorls of up to nine. Ecologically, S. antleroides is parasitic on the larvae of Noctuidae buried in soil and is different from S. cristata and $S$. tortricidae, both of which parasitize the pupae of Saturniidae in cocoons buried in soil and the pupae of Tortricidae in cocoons rolled in fallen leaves, respectively.

Samsoniella cardinalis H. Yu, Y.B. Wang, Y. Wang, Q. Fan \& Zhu L. Yang, sp. nov.

Mycobank: MB 833112; Fig. 12

Etymology: Referring to the scarlet stromata arising from the host in cocoons.

\section{Holotype: YHH 15732}

Sexual morph: Several stromata arising from oval cocoons of insect host, scarlet, cylindrical, $11.5-18.6 \mathrm{~mm}$ long. Stipes reddish-orange, $0.4-1.8 \mathrm{~mm}$ wide. Fertile parts clavate, ateral sides usually have a longitudinal ditch without producing perithecia, scarlet, $2.5-6.8 \times 0.5-2.6 \mathrm{~mm}$. Perithecia superficial, oblong-ovate to fusiform, 370-485 × 140-238 um. Asci eight-spored, hyaline, cylindrical $163-320 \times 3.2-4.3 \mu \mathrm{m}$. Ascus caps hemispherical, 1.9-3.0 $\times 1.4-2.6 \mu \mathrm{m}$. Ascospores hyaline, bola-shaped, septate, $165-230 \times 0.5-0.9 \mu \mathrm{m}$, central part filiform, terminal part narrowly fusiform, do not disarticulate into part-spores. Asexual morph: Isaria-like. Colonies on PDA growing fairly well at $25^{\circ} \mathrm{C}, 35-38 \mathrm{~mm}$ in 14 days, floccose, crater-shaped, white to pale pink, sporulating abundantly at the centrum. Reverse pale yellow to reddishbrown. Hyphae smooth-walled, branched, septate, hyaline, 1.3-2.2 $\mu \mathrm{m}$ wide. Conidiophores cylindrical, solitary or verticillate, $3.1-9.5 \times 1.3-2.0 \mu \mathrm{m}$. Phialides verticillate, in whorls of two to five, sometimes solitary on hyphae, basal portion cylindrical to narrowly lageniform, tapering gradually or abruptly toward the apex; $4.1-43.5 \mu \mathrm{m}$ long, 1.3-2.4 $\mu \mathrm{m}$ wide at the base, and $0.6-1.2 \mu \mathrm{m}$ wide at the apex. Conidia one-celled, smooth-walled, hyaline, fusiform or oval, 2.4-3.2 $\times 1.4-2.2 \mu \mathrm{m}$, often in chains.

Host: Pupae of Limacodidae.

Habitat: On the pupae of Limacodidae in cocoons buried in soil.

Distribution: Kunming City, China.

Material examined: CHINA. YUNNAN PROVINCE: Kunming City, Wild Duck Lake Forest Park, on the pupa of Limacodidae in a cocoon buried in soil, 12 August 2017, Hong Yu, (YHH 15732, holotype; YFCC 6144, ex-holotype living culture). Kunming City, Xishan Forest Park, on the pupa of Limacodidae in a cocoon buried in soil, 13 August 2018, Qi Fan, (YHH 15764; YFCC 6320, living culture). VIETNAM. LAOCAI PROVINCE: Sapa County, Hoang
Lien Mountains, on the pupa of Limacodidae in a cocoon buried in soil, 26 October 2016, Yuan-Bing Wang, (YHH 14891; YFCC 5830, living culture).

Notes: Samsoniella cardinalis is characterized by scarlet stromata with clavate fertile parts, superficial perithecia, oblong-ovate to fusiform, cylindrical asci, bola-shaped ascospores, crater-shaped colonies with white to pale pink, having Isaria-like asexual conidiogenous structure, and on the pupae of Limacodidae in cocoons buried in soil.

It is similar to $S$. alpina in sharing Isaria-like asexual conidiogenous structure which produces phialides with cylindrical to narrowly lageniform basal portion, fusiform or oval conidia. However, it differs from S. alpina by its scarlet stromata with clavate fertile parts and superficial perithecia, crater-shaped colonies with white to pale pink colors, and longer phialides up to $43.5 \mu \mathrm{m}$. Ecologically, it is parasitic on the pupae of Limacodidae in cocoons and is significantly different from S. alpina which parasitizes the larvae of $H$. baimaensis (Hepialidae).

Samsoniella cristata H. Yu, Y.B. Wang, Y. Wang, Q. Fan \& Zhu L. Yang, sp. nov.

Mycobank: MB 833113; Fig. 13

Etymology: Referring to the crista-like stromata.

\section{Holotype: YHH 16982}

Sexual morph: Stromata arising from the insect cocoons, solitary or two, much branched, $25-40 \mathrm{~mm}$ long, orange, crista-like. Stipes fleshly, white at the rhizine, becoming orange towards the upper part, $1.0-1.5 \mathrm{~mm}$ wide. Fertile parts reddish orange, crista-like or subulate, $3.1-18.5 \times 0.9-8.0 \mathrm{~mm}$. Perithecia crowded, superficial, narrowly ovoid, 370-485 $\times 150-245 \mu \mathrm{m}$. Asci eightspored, hyaline, cylindrical $180-356 \times 3.0-4.8 \mu \mathrm{m}$. Ascus caps hemispherical, $2.7-3.8 \times 1.5-2.4 \mu \mathrm{m}$. Ascospores hyaline, bola-shaped, septate, $155-290 \times 1.0-1.3 \mu \mathrm{m}$, central part filiform, terminal part narrowly fusiform, do not disarticulate into part-spores. Asexual morph: Isarialike. Colonies on PDA fast-growing, 43-50 mm diameter in 14 days at $25^{\circ} \mathrm{C}$, floccose, crater-shaped, white to light orange, forming yellow-brown concentric rings around the inoculum, sporulating abundantly, cottony, with high mycelial density around the edge, reverse pale brown. Hyphae smooth-walled, branched, septate, hyaline, 1.3-2.5 $\mu \mathrm{m}$ wide. Conidiophores smooth-walled, cylindrical, solitary or verticillate, $3.6-11.5 \times 1.7-2.5 \mu \mathrm{m}$. Phialides verticillate, in whorls of two to five, usually solitary on hyphae, basal portion cylindrical to narrowly lageniform, tapering gradually or abruptly toward the apex; $4.5-23.2 \mu \mathrm{m}$ long, 1.6-2.7 $\mu \mathrm{m}$ wide at the base, and $0.5-1.1 \mu \mathrm{m}$ wide at the apex. Conidia one-celled, smooth-walled, hyaline, fusiform or oval, 2.4-3.2 $\times 1.6-2.3 \mu \mathrm{m}$, often in chains.

Host: Pupae of Saturniidae.

Habitat: On the pupae of Saturniidae in cocoons buried in soil. 

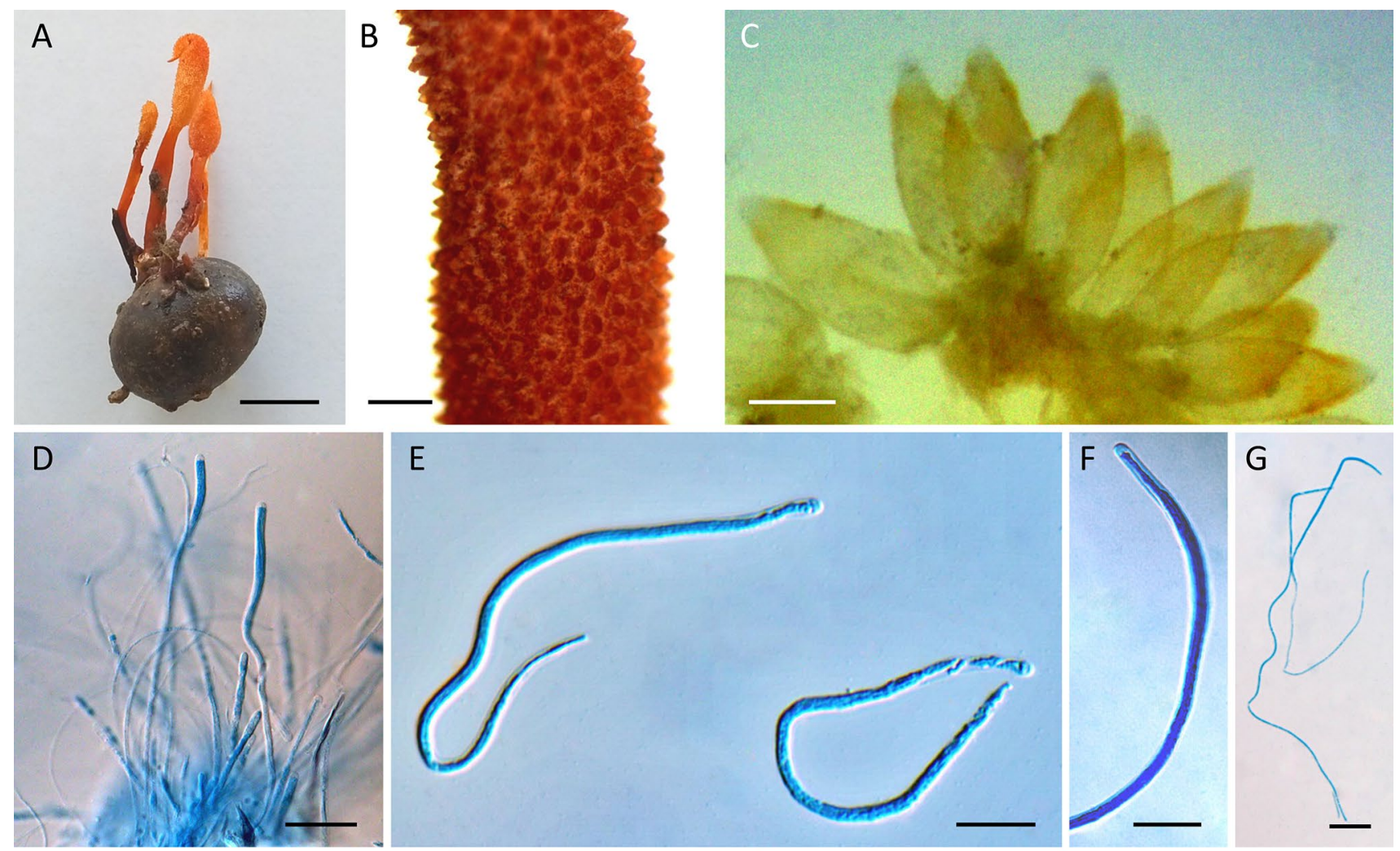

E
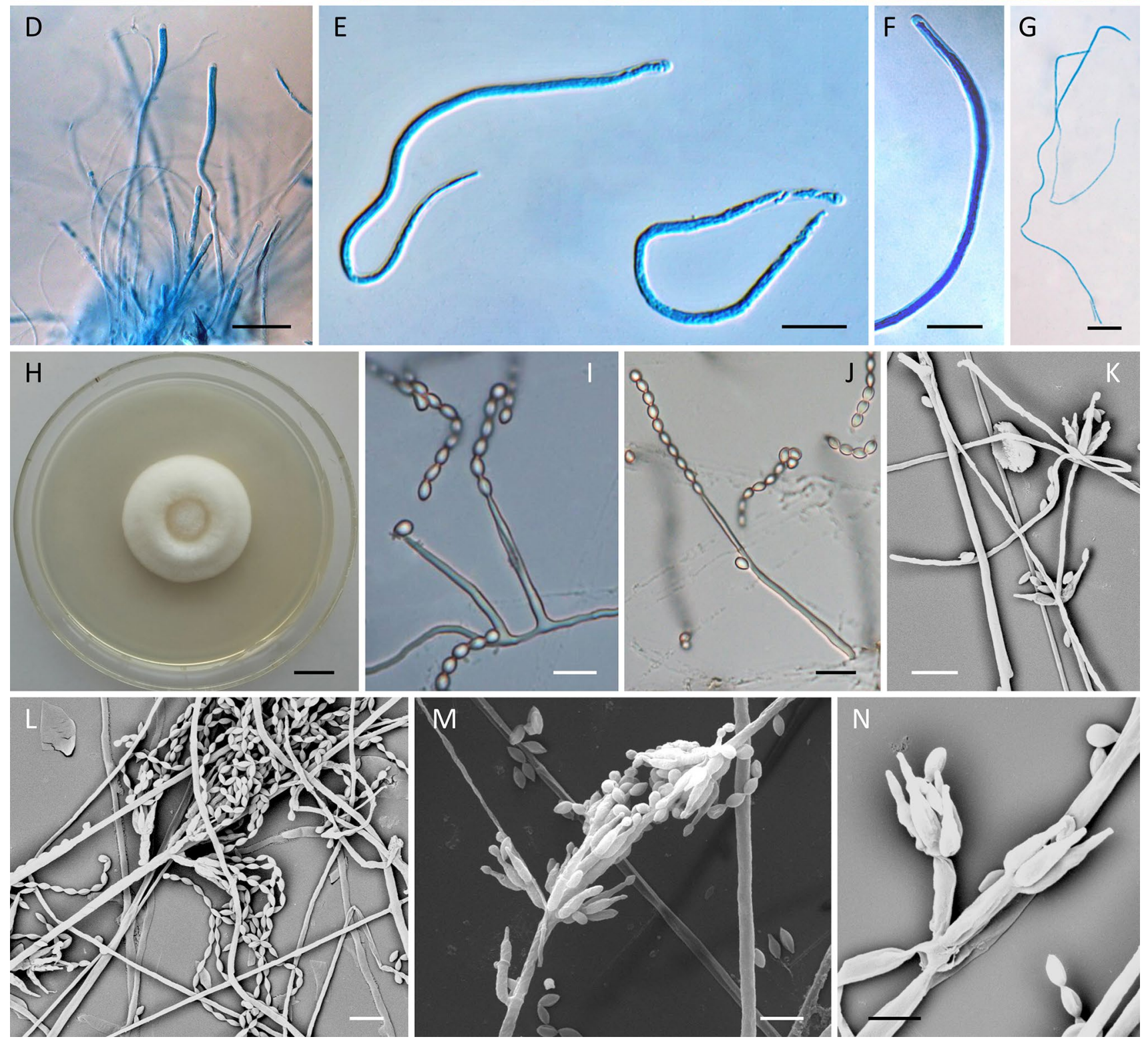

Fig. 12 Samsoniella cardinalis. A Fungus on the pupa of Limacodidae in a cocoon. B Fertile part. C Perithecia. D-F Asci. G Ascospores. H Colony on PDA. I, J Solitary phialides on hyphae.
$\mathbf{K}-\mathbf{N}$ Verticillate phialides on conidiophores. Scale bars: $\mathbf{A}=5 \mathrm{~mm}$; $\mathbf{B}, \mathbf{C}=200 \mu \mathrm{m} ; \mathbf{D}, \mathbf{E}=20 \mu \mathrm{m} ; \mathbf{F}, \mathbf{G}=10 \mu \mathrm{m} ; \mathbf{H}=1 \mathrm{~cm} ; \mathbf{I}-\mathbf{N}=5 \mu \mathrm{m}$ 

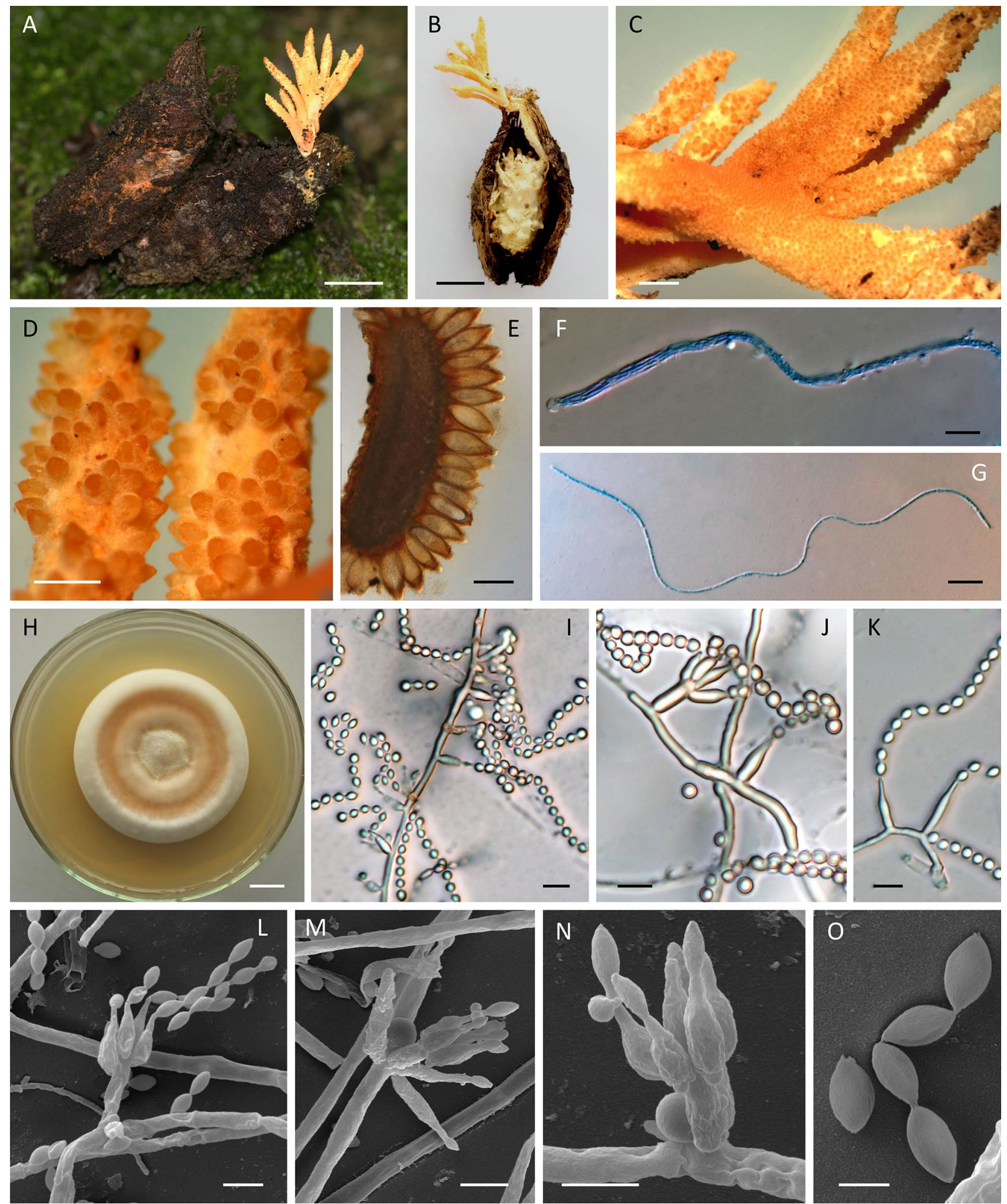

Fig. 13 Samsoniella cristata. A Crista-like stromata arising from the host. B Fungus on the pupa of Saturniidae in a cocoon. C, D Fertile parts. E Perithecia. F Asus. G Ascospore. H Colony on PDA. I Solitary phialides on hyphae. J Verticillate phialides on conidiophores.
K Solitary phialides on hyphae. L-N Verticillate phialides on conidiophores. O Conidia. Scale bars: A, $\mathbf{B}=1 \mathrm{~cm} ; \mathbf{C}, \mathbf{D}=500 \mu \mathrm{m}$; $\mathbf{E}=200 \mu \mathrm{m} ; \mathbf{F}=5 \mu \mathrm{m} ; \mathbf{G}=10 \mu \mathrm{m} ; \mathbf{H}=1 \mathrm{~cm} ; \mathbf{I}-\mathbf{N}=5 \mu \mathrm{m} ; \mathbf{O}=2 \mu \mathrm{m}$ 

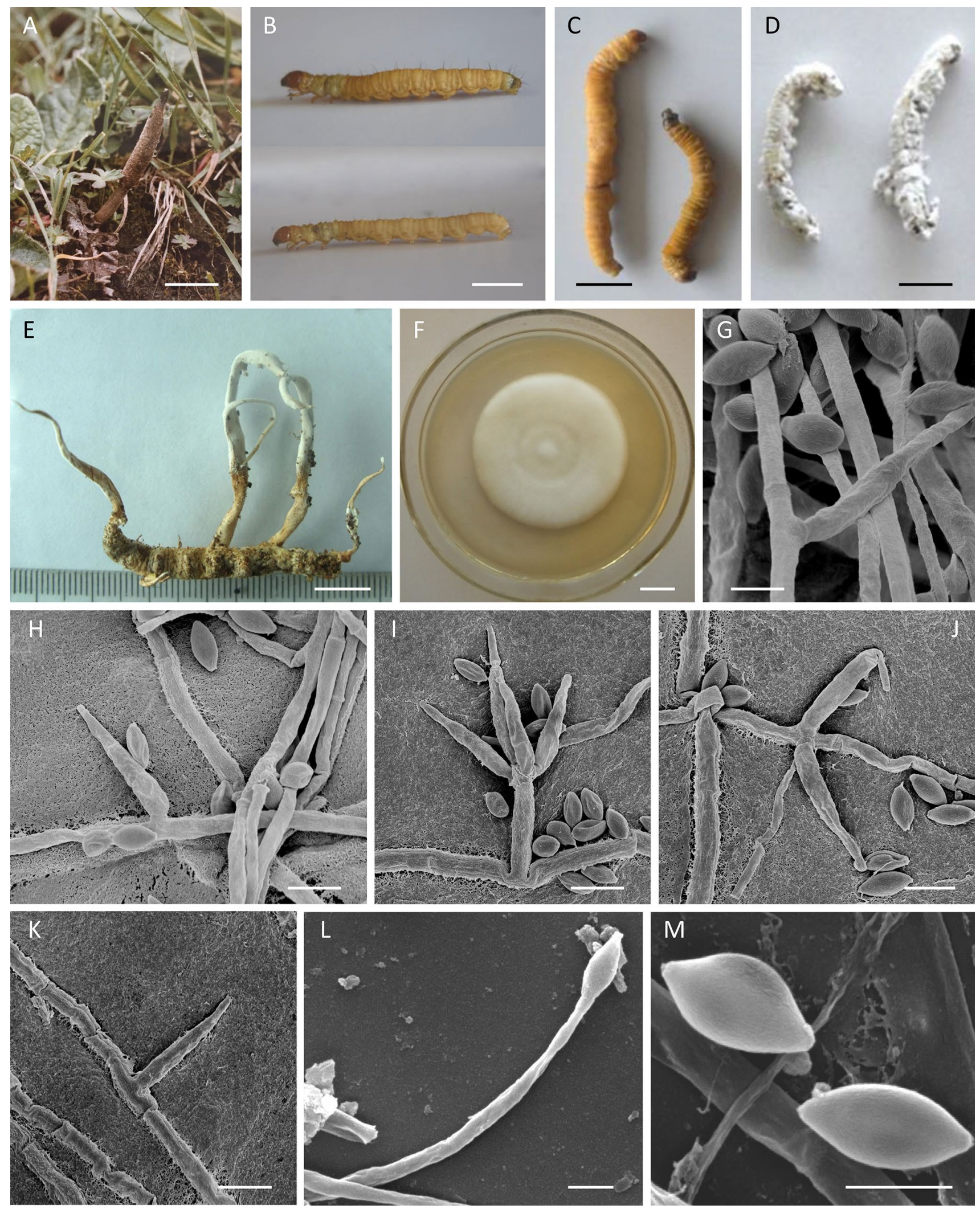
४Fig. 14 Samsoniella hepiali. A Stroma of Ophiocordyceps sinensis arising from the larva of Hepialus buried in soil in the Baima Snow Mountain, Yunnan Province (This picture taken in June 1982 was provided by Ru-Qin Dai); the holotype material of S. hepiali was isolated from this specimen. B The host larvae of Hepialus baimaensis. C, D Larvae of $H$. baimaensis infected by $S$. hepiali. E Synnemata arising from the whole body of $H$. yunnanensis. F Colony on PDA. G-J Verticillate phialides on conidiophores. K, L Solitary phialides on hyphae. M Conidia. Scale bars: $\mathbf{A}-\mathbf{F}=1 \mathrm{~cm} ; \mathbf{G}-\mathbf{K}=5 \mu \mathrm{m} ; \mathbf{L}$, $\mathbf{M}=2 \mu \mathrm{m}$

\section{Distribution: Kunming City, China.}

Material examined: CHINA. YUNNAN PROVINCE: Kunming City, Wild Duck Lake Forest Park, on the pupa of Saturniidae in a cocoon buried in soil, 14 August 2018, Hong Yu, (YHH 16982, holotype; YFCC 7004, ex-holotype living culture); Ibid. (YHH 15760, paratype; YFCC 6021, ex-paratype living culture); Ibid. (YHH 15761, paratype; YFCC 6023, ex-paratype living culture).

Notes: Samsoniella cristata is characterized by solitary or two stromata, crista-like, reddish orange fertile parts, superficial and narrowly ovoid perithecia, cylindrical asci, bola-shaped ascospores, crater-shaped colonies with white to light orange colors, Isaria-like asexual conidiogenous structure, and on the pupae of Saturniidae in cocoons.

Phylogenetically, S. cristata is sister to $S$. tortricidae, a novel species described in this study. Samsoniella cristata resembles $S$. tortricidae in sharing stromata with terminal branches, subulate fertile parts, and Isaria-like asexual conidiogenous structure. However, it differs in having fewer and shorter stromata, crista-like, crater-shaped colonies, shorter phialides, and it is parasitic on the pupae of Saturniidae in cocoons buried in soil.

Samsoniella hepiali (Q.T. Chen \& R.Q. Dai ex R.Q. Dai et al.) H. Yu, R.Q. Dai, Y.B. Wang, Y. Wang \& Zhu L. Yang, comb. nov.

Mycobank: MB 833114; Fig. 14

Basionym: Paecilomyces hepiali Q.T. Chen \& R.Q. Dai ex R.Q. Dai et al., Mycosystema 27 (5): 642 (2008); Paecilomyces hepiali Q.T. Chen \& R.Q. Dai, Acta Agric. Univ. Pekin. 6(2): 223 (1989), invalid.

\section{Holotype: IMM 82-2 [as “CHICMM 82-2”]}

Sexual morph: Undetermined. Asexual morph: Isarialike. Synnemata arising from the whole body of lepidopteran insects, branched or unbranched, $0.5-4.1 \mathrm{~cm}$ long. Stipes cylindrical or clavate, $0.6-3.2 \mathrm{~mm}$ wide, with powdery conidia at the apex, white to yellowish. Conidiophores along the apex of synnemata, solitary, with phialides in whorls of two to five, cylindrical, 4.1-7.3×1.4-2.0 $\mu \mathrm{m}$. Phialides with a basal portion cylindrical to narrowly lageniform, 4.5-12.6×1.5-2.6 $\mu \mathrm{m}$, tapering gradually or abruptly into a narrow neck of $0.5-1.0 \mu \mathrm{m}$ wide. Conidia in chains, one-celled, smooth-walled, hyaline, fusiform or oval, 2.0-3.1 $\times 1.4-1.9 \mu \mathrm{m}$. Colonies on PDA moderately fast-growing, 50-55 mm diameter in 14 days at $25^{\circ} \mathrm{C}$, cottony, with high mycelial density, white to yellowish, forming concentric rings around the inoculum. Reverse white to yellowish, turning orange when old. Hyphae smooth-walled, branched, septate, hyaline, 1.3-2.2 $\mu \mathrm{m}$ wide. Conidiophores smooth-walled, cylindrical, solitary, 4.0-7.6×1.4-2.2 $\mu \mathrm{m}$. Phialides on conidiophores verticillate, in whorls of two to five, solitary or opposite on hyphae, basal portion cylindrical to narrowly lageniform, tapering gradually or abruptly toward the apex; 3.5-13.6 $\mu \mathrm{m}$ long, 1.3-2.1 $\mu \mathrm{m}$ wide at the base, $0.5-1.0 \mu \mathrm{m}$ wide at the apex. Conidia one-celled, smoothwalled, hyaline, fusiform or oval, 1.8-3.3 $\times 1.4-2.2 \mu \mathrm{m}$, often in chains.

Host: Larvae and pupae of Lepidoptera.

Habitat: On the larvae and pupae of Lepidoptera, the larvae of Hepialus parasitized by $O$. sinensis buried in soil or clinging to fallen leaves.

Distribution: Deqin County, Shangrila City, Huanglong County, China; Sapa County, Vietnam.

Material examined: CHINA. YUNNAN PROVINCE: Diqing Tibetan Autonomous Prefecture, Deqin County, Baima Snow Mountain, isolated from the larva of $H$. armoricanus parasitized by $O$. sinensis, June 1982, Ru-Qin Dai, (IMM 82-2 = CHICMM 82-2, holotype; ICMM 82-2, exholotype living culture); same location, isolated from the larva of $H$. baimaensis associated with $O$. sinensis, May 2002, Zhuo Zhang, (YHH 1056; YFCC 661, living culture); same location, on the larva of $H$. baimaensis associated with O. sinensis, 26 May 2010, Hong Yu, (YHH 4258; YFCC 2702, living culture); same location, associated with $O$. sinensis on the larva of $H$. baimaensis, 18 May 2018, Hong Yu, (YHH 16883; YFCC 7024, living culture); Diqing Tibetan Autonomous Prefecture, Shangrila City, Xiaozhongdian Town, on the larva of H. yunnanensis, 24 December 2017, Hong Yu, (YHH 16827; YFCC 7215, living culture). QINHAI PROVINCE: Haidong City, Huanglong County, (Cor4, dried culture). VIETNAM. LAOCAI PROVINCE: Sapa County, Hoang Lien Mountains, on the pupa of Lepidoptera buried in soil, 26 October 2016, Yuan-Bing Wang, (YHH 14896; YFCC 5823, living culture); same location, on the larva of Lepidoptera clinging to fallen leaves, 26 October 2016, Yao Wang, (YHH 14898; YFCC 5828, living culture).

Notes: This fungus, named as Paecilomyces hepiali by Dai et al. (1989), was originally collected from the Baima Snow Mountain in Yunnan Province, China based on isolates from the larvae of $H$. armoricanus parasitized by $O$. sinensis. However, the name was effectively, but not formally published due to the failure of the authors to comply with the requirements of the Code for type indication, and the only cited material was a living culture (Dai et al. 2008; Turland et al. 2018). Paecilomyces hepiali was later validly published and the holotype IMM 82-2 was designated using a dried culture from the living culture 82-2 (ICMM 82-2) (Dai et al. 2008, 2018a). 
Based on the original description, $P$. hepiali was morphologically similar to $P$. xylariiformis (Lloyd) Samson, originally named as I. xylariiformis Lloyd, but it differs in the globose or subglobose conidia with smaller size and the host of hepialid larvae (Dai et al. 1989). In addition, $P$. hepiali differed from I. farinosa (Holmsk.) Fr., currently recombined into $C$. farinosa, by the shape and arrangement of phialides, the shape of conidia, its host belonging to the genus Hepialus, and its habitat of an extremely cold area at an altitude of 4000-4500 m (Dai et al. 1989).

Paecilomyces xylariiformis, probably belonging to Isaria, is only known from dried type herbarium material no. 42613, and its phylogenetic analyses have not been conducted (Samson 1974; Luangsa-ard et al. 2005). Its generic status, and even so, higher taxonomic rank remain unresolved. Recent phylogenetic analyses together with our fivegene phylogeny of the family Cordycipitaceae show that $C$. farinosa belongs to the type genus Cordyceps of this family (Kepler et al. 2017; Mongkolsamrit et al. 2018). In our phylogenetic analyses, the holotype material ICMM 82-2 and seven other samples of $P$. hepiali were used to determined its systematic position. Our results show that the eight samples of $P$. hepiali group together with strong statistical support (BP $=80 \%$ and $\mathrm{PP}=100 \%$ ), are clustered within the recently established genus Samsoniella of Cordycipitaceae, and form a single clade related to an undescribed taxon Isaria sp. TNS 16333 (Fig. 1, 2). Consequently, P. hepiali is phylogenetically distinguished from $C$. farinosa which also produces Isaria-like asexual morph. Based on the strong phylogenetic and morphological evidence, a new combination, namely $S$. hepiali is proposed for P. hepiali.

Here, a redescription of $S$. hepiali is made on the basis of morphological observations of the ex-holotype living culture ICMM 82-2 and related samples collected in this study. Samsoniella hepiali has Isaria-like asexual morph and is characterized by branched or unbranched synnemata arising from the whole body of lepidopteran insects, cylindrical or clavate stipes with a powdery conidia at the apex, white to yellowish, moderately fast-growing colonies with white to yellowish colors, cottony, solitary conidiophores with cylindrical shape, solitary or verticillate phialides with cylindrical to narrowly lageniform basal portion, fusiform or oval conidia often in chains.

Samsoniella hepiali is morphologically similar to $S$. alpina and $S$. yunnanensis in the Isaria-like asexual conidiogenous structure, producing synnemata with powdery conidia at the apex. However, $S$. hepiali differs from $S$. alpina by its white to yellowish colonies, solitary conidiophores with phialides in whorls of two to five and longer phialides. It differs from $S$. yunnanensis because the latter has synnemata with orange to pink stipes, white colonies, solitary or verticillate conidiophores up to $23.5 \mu \mathrm{m}$ long with phialides in whorls of two to seven. Ecologically, S. hepiali and S. alpina share similar host larvae of Hepialus, whereas $S$. hepiali has a wider lepidopteran species host range.

Samsoniella kunmingensis $\mathrm{H}$. Yu, Y.B. Wang, Y. Wang, Q. Fan \& Zhu L. Yang, sp. nov.

Mycobank: MB 833116; Fig. 15

Etymology: Named after the location Kunming City where the species was collected.

Holotype: YHH 6002

Sexual morph: Stromata arising from the lepidopteran pupa buried in soil, solitary, up to $23 \mathrm{~mm}$ long, cylindrical to clavate, bifurcated. Stipes fleshly, white to orange, 0.5-0.9 mm wide, with a terminal bifurcated branch of 5.2-11.4 mm long. Fertile parts reddish orange, clavate, ateral sides usually have a longitudinal ditch without producing perithecia, 3.3-4.2 $\times 0.8-1.2 \mathrm{~mm}$. Perithecia crowded, superficial, narrowly ovoid to fusiform, 330-395 × 110-185 $\mu \mathrm{m}$. Asci eight-spored, hyaline, cylindrical, 150-297 ×3.0-4.6 $\mu \mathrm{m}$. Ascus caps hemispherical, 2.3-3.6 $\times 1.5-2.5 \mu \mathrm{m}$. Ascospores hyaline, bola-shaped, septate, $127-190 \times 0.8-1.5 \mu \mathrm{m}$, central part filiform, terminal part narrowly fusiform, do not disarticulate into part-spores. Asexual morph: Undetermined.

Host: Pupa of Lepidoptera.

Habitat: On the pupa of Lepidoptera buried in soil.

Distribution: Kunming City, China.

Material examined: CHINA. YUNNAN PROVINCE: Kunming City, Wild Duck Lake Forest Park, on the pupa of Lepidoptera buried in soil, 12 August 2017, Qi Fan, (YHH 16002, holotype).

Notes: Samsoniella kunmingensis is characterized by solitary stromata, bifurcated, clavate fertile parts with reddish orange color, ateral sides usually have a longitudinal ditch without producing perithecia, superficial perithecia, narrowly ovoid to fusiform, and cylindrical asci with bolashaped ascospores.

Phylogenetically, it is closely related to the new species S. ramosa described in this study. However, S. kunmingensis is morphologically similar to $S$. antleroides, S. lanmaoa and $S$. inthanonensis by producing superficial perithecia, narrowly ovoid to fusiform, cylindrical asci with bola-shaped ascospores. Samsoniella kunmingensis differs from S. antleroides, S. lanmaoa, S. inthanonensis and $S$. ramosa by its solitary and bifurcated stromata, and clavate fertile parts with reddish orange color. The latter three species have Isaria-like asexual morphs, whereas asexual morph of $S$. kunmingensis was not determined in this study.

Samsoniella lanmaoa H. Yu, Y.B. Wang, Y. Wang, Q. Fan \& Zhu L. Yang, sp. nov.

Mycobank: MB 833115; Fig. 16

Etymology: Lanmaoa is named for Mr. Lan Mao (1397-1476, Ming Dynasty), an ancient Chinese botanist, who recorded medicinal fungi in the famous Chinese literature "Dian Nan Ben Cao". 

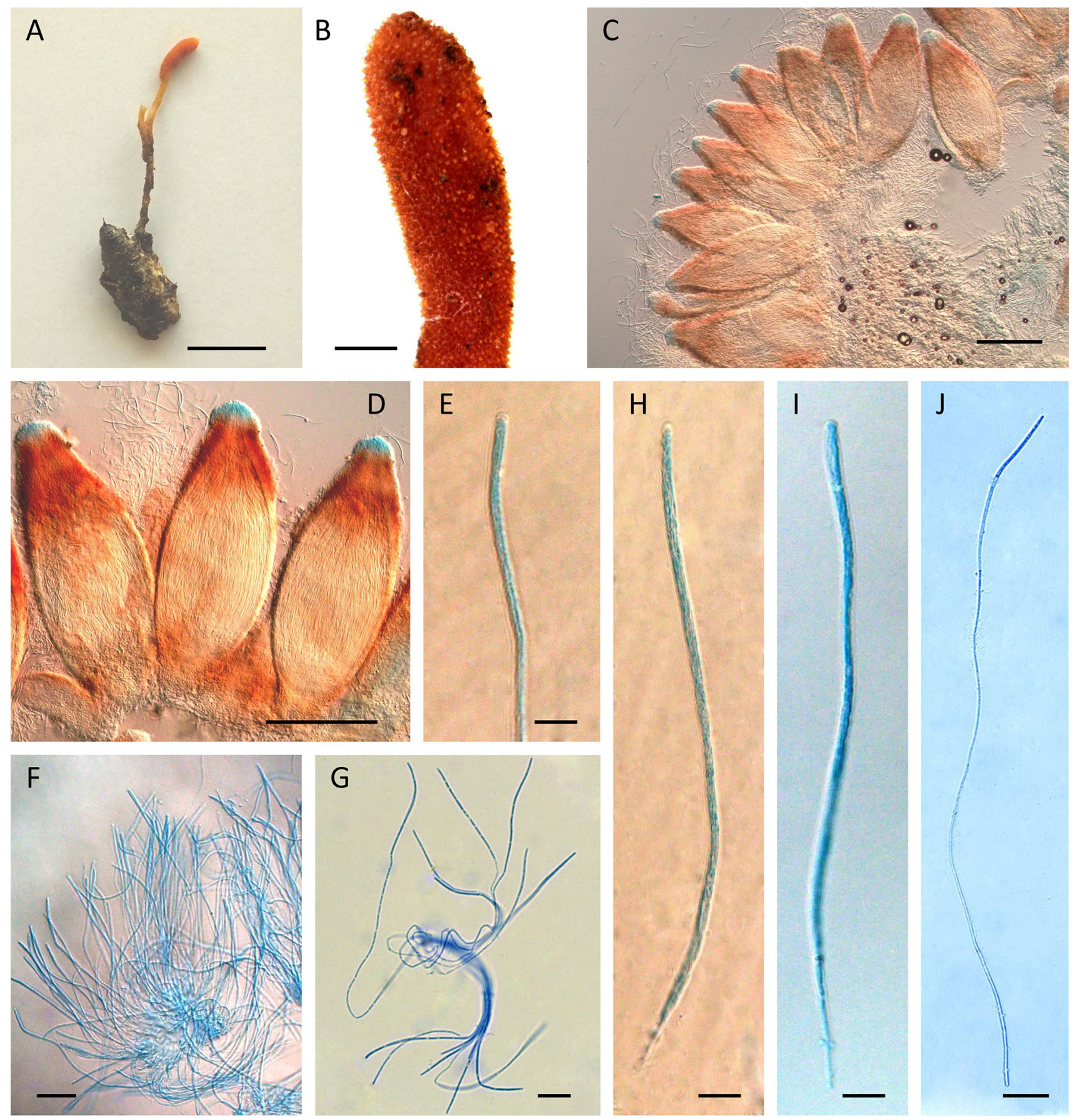

Fig. 15 Samsoniella kunmingensis. A Fungus on the pupa of Lepidoptera. B Fertile parts. C, D Perithecia. E, F Asci. G Ascospores. H, I Asci. J Ascospore. Scale bars: $\mathbf{A}=1 \mathrm{~cm} ; \mathbf{B}=500 \mu \mathrm{m} ; \mathbf{C}, \mathbf{D}=200 \mu \mathrm{m} ; \mathbf{E}=5 \mu \mathrm{m} ; \mathbf{F}=20 \mu \mathrm{m} ; \mathbf{G}-\mathbf{I}=5 \mu \mathrm{m} ; \mathbf{J}=10 \mu \mathrm{m}$

Holotype: YHH 15740

Sexual morph: Stromata arising from insect cocoons, two to five, 38-69 $\mathrm{mm}$ long, palmately branched, orange. Stipes fleshly, clavate, palmated at the branching portion, 1.2-3.9 mm wide. Fertile parts reddish orange, clavate, ateral sides usually have a longitudinal ditch without producing perithecia, $8.5-11.2 \times 0.6-2.3 \mathrm{~mm}$.
Perithecia crowded, superficial, narrowly ovoid to fusiform, 360-467× 124-210 $\mu \mathrm{m}$. Asci eight-spored, hyaline, cylindrical, 160-325 ×3.3-4.8 $\mu \mathrm{m}$. Ascus caps hemispherical, 2.5-3.8 $\times 1.6-2.3 \mu \mathrm{m}$. Ascospores hyaline, bola-shaped, septate, $135-260 \times 0.9-1.4 \mu \mathrm{m}$, central part filiform, terminal part narrowly fusiform, do not disarticulate into partspores. Asexual morph: Isaria-like. Colonies on PDA 

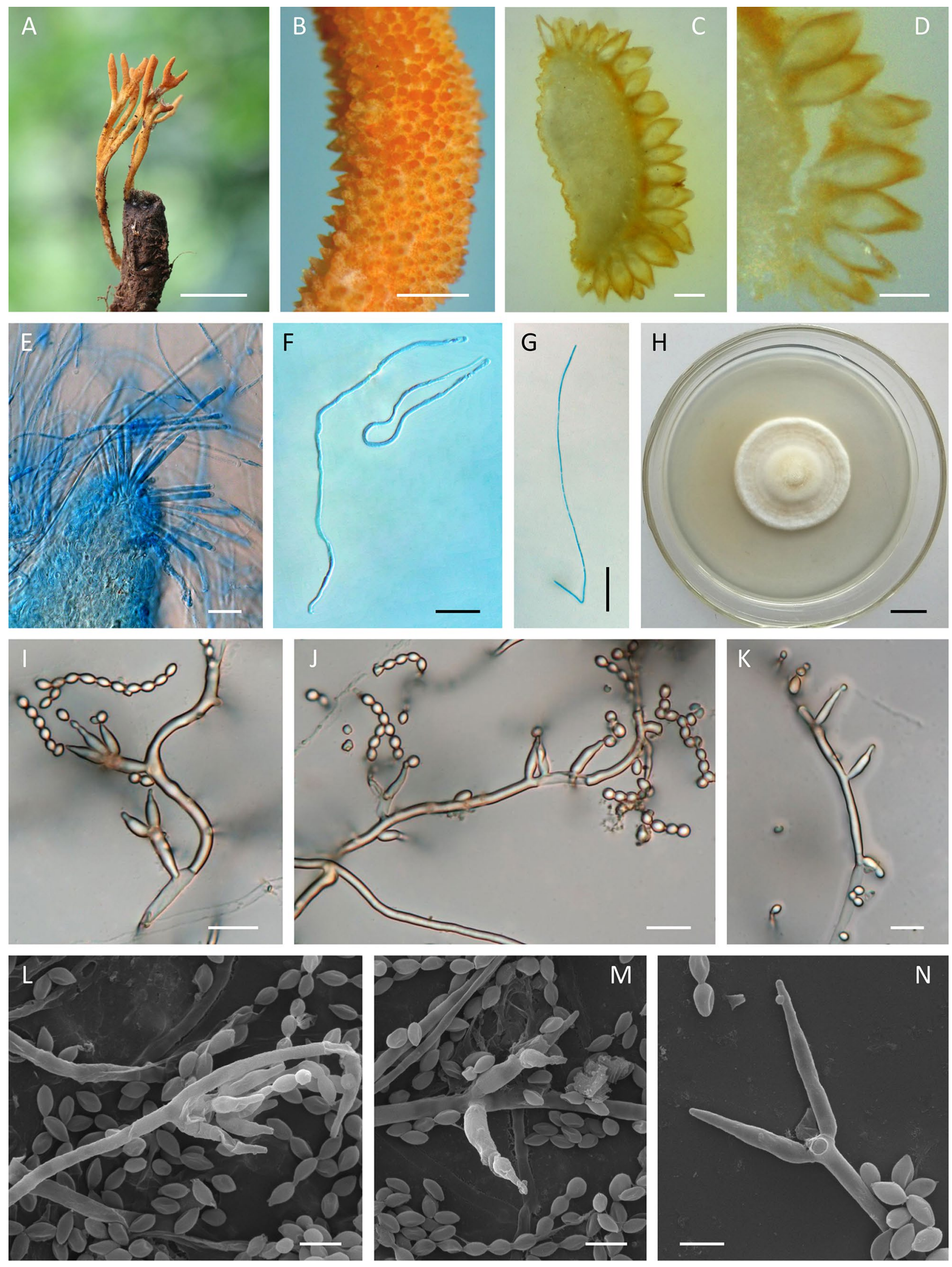
4Fig. 16 Samsoniella lanmaoa. A Stromata arising from the pupa of Lepidoptera. B Fertile parts. C, D Perithecia. E-F Asci. G Ascospore. H Colony on PDA. I, J Verticillate phialides on conidiophores. K Solitary phialides on hypha. L, N Verticillate phialides on conidiophores. Scale bars: $\mathbf{A}=1 \mathrm{~cm} ; \mathbf{B}=500 \mu \mathrm{m} ; \mathbf{C}-\mathbf{E}=200 \mu \mathrm{m}$; $\mathbf{E}-\mathbf{G}=20 \mu \mathrm{m} ; \mathbf{H}=1 \mathrm{~cm} ; \mathbf{I}-\mathbf{N}=5 \mu \mathrm{m}$

fast-growing, $38-40 \mathrm{~mm}$ diameter in 14 days at $25^{\circ} \mathrm{C}$, white to cream-colored, cottony, with high mycelial density at the centrum, forming concentric rings around the inoculum, reverse pale yellow to yellowish-brown. Hyphae smooth-walled, branched, septate, hyaline, 1.2-1.9 $\mu \mathrm{m}$ wide. Conidiophores smooth-walled, cylindrical, solitary or verticillate, $3.8-13.3 \times 1.5-2.1 \mu \mathrm{m}$. Phialides verticillate, in whorls of two to six, usually solitary on hyphae, basal portion cylindrical to narrowly lageniform, tapering gradually or abruptly toward the apex; 3.5-20.7 $\mu \mathrm{m}$ long, $1.7-2.6 \mu \mathrm{m}$ wide at the base, and $0.5-1.1 \mu \mathrm{m}$ wide at the apex. Conidia one-celled, smooth-walled, hyaline, fusiform or oval, 1.9-2.7×1.4-2.0 $\mu \mathrm{m}$, often in chains.

Host: Pupae of Lepidoptera.

Habitat: On the pupae of Lepidoptera in cocoons buried in soil.

Distribution: Kunming City, China.

Material examined: CHINA. YUNNAN PROVINCE: Kunming City, Wild Duck Lake Forest Park, on the pupa of Lepidoptera in a cocoon buried in soil, 12 August 2017, Hong Yu, (YHH 15740, holotype; YFCC 6148, ex-holotype living culture); Ibid., (YHH 15753, paratype; YFCC 6193, ex-paratype living culture).

Notes: Samsoniella lanmaoa is characterized by palmately branched stromata, clavate fertile parts with reddish orange color, superficial perithecia with narrowly ovoid to fusiform shapes, bola-shaped ascospores, white to creamcolored colonies, and Isaria-like asexual conidiogenous structure.

Phylogenetic analyses show that S. lanmaoa is sister to the recently described species $S$. inthanonensis, by which the genus Samsoniella was established. Morphologically, S. lanmaoa is similar to $S$. inthanonensis in producing branched stromata with reddish to orange colors, bola-shaped ascospores and Isaria-like asexual conidiogenous structure (Mongkolsamrit et al. 2018). However, it differs from $S$. inthanonensis by its longer stromata $(38-69 \mathrm{~mm})$, narrowly ovoid to fusiform perithecia, white to cream-colored colonies and larger phialides (3.5-20.7 $\mu \mathrm{m}$ long). Ecologically, $S$. lanmaoa is parasitic on the lepidopteran pupae in cocoons buried in soil, whereas $S$. inthanonensis was reported as a parasite of the lepidopteran larvae in leaf litter (Mongkolsamrit et al. 2018).

Samsoniella ramosa H. Yu, Y.B. Wang, Y. Wang, Q. Fan \& Zhu L. Yang, sp. nov.

Mycobank: MB 833117; Fig. 17
Etymology: Referring to the ramose stromata.

Holotype: YHH 15988

Sexual morph: Stromata arising directly from an insect cocoon, fascicular, multi-branched, often confluent at the base $15-32 \times 0.8-1.5 \mathrm{~mm}$. Stipes oblate or flaky, white at first, then turning into fulvous, rusty-brown when old. Fertile parts having no obvious boundary with stipes, white to pale brown, with a tapering sterile part, 1.6-7.8 mm long, white to khaki. Perithecia crowded, superficial, narrowly ovoid to fusiform, $340-435 \times 130-197 \mu \mathrm{m}$. Asci and ascospores not observed. Asexual morph: Isaria-like. A mass of conidia producing toward the apex of stromatic branches, white, powdery and floccose. Conidiophores on the apex of stromata with phialides in whorls of two to five, cylindrical, 5.1-12.3 $\times 1.4-2.5 \mu \mathrm{m}$. Phialides with a basal portion cylindrical to narrowly lageniform, $4.5-12.6 \times 1.5-2.6 \mu \mathrm{m}$, tapering abruptly into a narrow neck of $0.5-1.1 \mu \mathrm{m}$ wide. Conidia in chains, one-celled, smooth-walled, hyaline, fusiform or oval, $2.0-3.3 \times 1.4-2.0 \mu \mathrm{m}$. Colonies on PDA moderately fast-growing, $45-50 \mathrm{~mm}$ diameter in 14 days at $25{ }^{\circ} \mathrm{C}$, floccose, crater-shaped, white to cream-coloured, sporulating abundantly, reverse off-white. Hyphae smoothwalled, branched, septate, hyaline, 1.5-2.8 $\mu \mathrm{m}$ wide. Conidiophores smooth-walled, cylindrical, solitary or verticillate, 4.3-10.5 × 1.3-2.4 $\mu \mathrm{m}$. Phialides verticillate, in whorls of two to six, usually solitary on hyphae, basal portion cylindrical to narrowly lageniform, tapering gradually or abruptly toward the apex; 5.3-14.6 $\mu \mathrm{m}$ long, $1.3-2.8 \mu \mathrm{m}$ wide at the base, and $0.6-1.2 \mu \mathrm{m}$ wide at the apex. Conidia one-celled, smooth-walled, hyaline, fusiform or oval, 2.0-3.6 $\times 1.5-2.2 \mu \mathrm{m}$, often in chains.

Host: Pupa of Limacodidae.

Habitat: On the pupa of Limacodidae in a cocoon buried in soil.

Distribution: Kunming City, China.

Material examined: CHINA. YUNNAN PROVINCE: Kunming City, Wild Duck Lake Forest Park, on the pupa of Limacodidae in a cocoon buried in soil, 12 August 2017, Hong Yu, (YHH 15988, holotype; YFCC 6020, ex-holotype living culture).

Notes: Samsoniella ramosa is characterized by fascicular stromata, multi-branched, oblate or flaky stipes, fertile parts with no obvious boundary with stipes, superficial perithecia, narrowly ovoid to fusiform, floccose and crater-shaped colonies, having Isaria-like asexual conidiogenous structure, and on the pupa of Limacodidae in a cocoon buried in soil.

It is similar to its phylogenetically closely related species $S$. kunmingensis in producing superficial perithecia, narrowly ovoid to fusiform. However, $S$. ramosa is easily distinguished by its fascicular stromata, multi-branched, oblate or flaky stipes and fertile parts having no obvious boundary with stipes. 

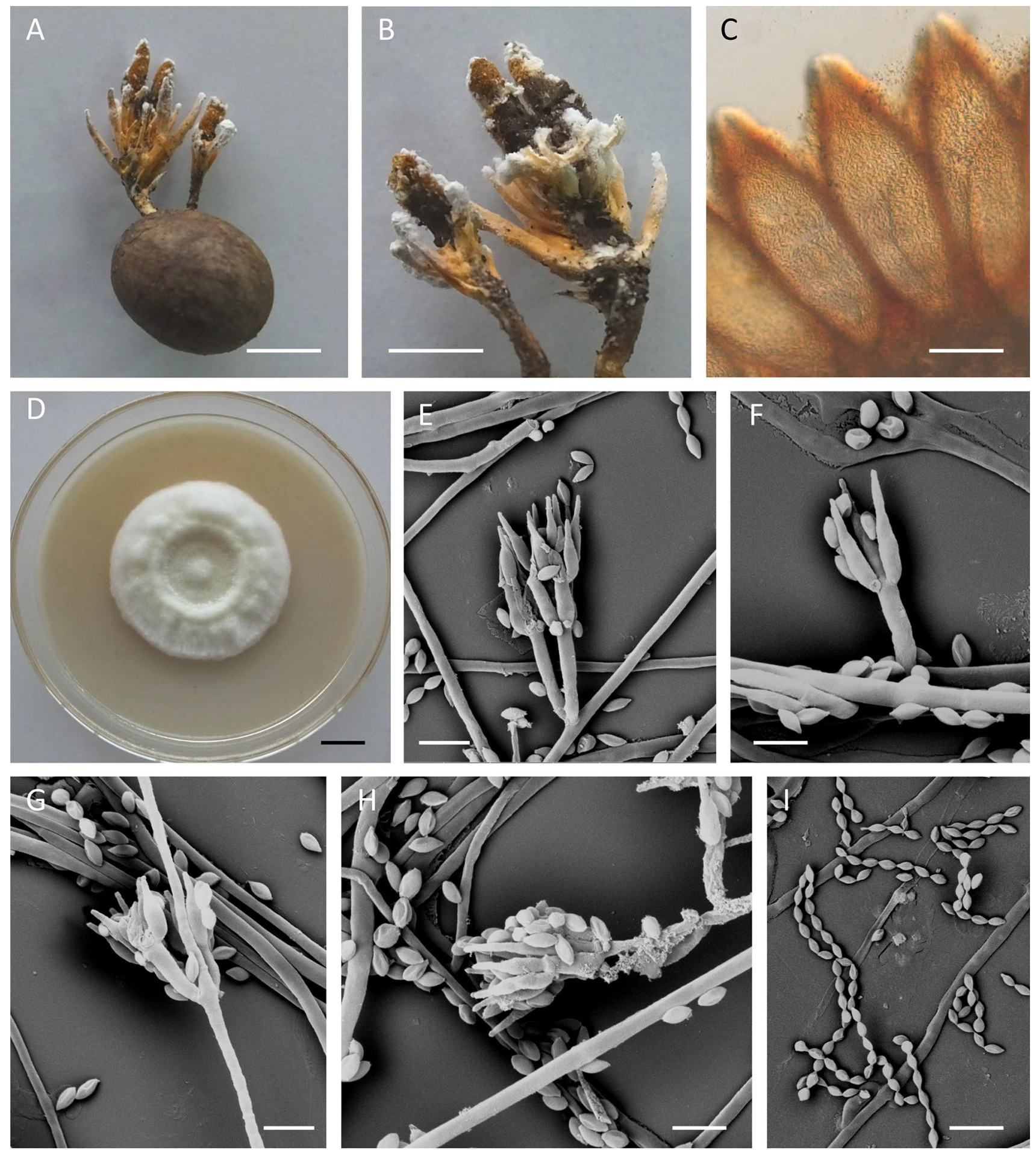

Fig. 17 Samsoniella ramosa. A Fungus on the pupa of Limacodidae in a cocoon. B Ramose stromata. C Perithecia. D Colony on PDA. E-H Verticillate phialides on conidiophores. I Conidia in chains. Scale bars: $\mathbf{A}, \mathbf{B}=5 \mathrm{~mm} ; \mathbf{C}=200 \mu \mathrm{m} ; \mathbf{D}=1 \mathrm{~cm} ; \mathbf{E}-\mathbf{I}=5 \mu \mathrm{m}$

Samsoniella tortricidae H. Yu, Y.B. Wang, Y. Wang, Q. Fan \& Zhu L. Yang, sp. nov.

Mycobank: MB 833118; Fig. 18
Etymology: Named after the host belonging to the family Tortricidae (Lepidoptera).

Holotype: YHH 16050 

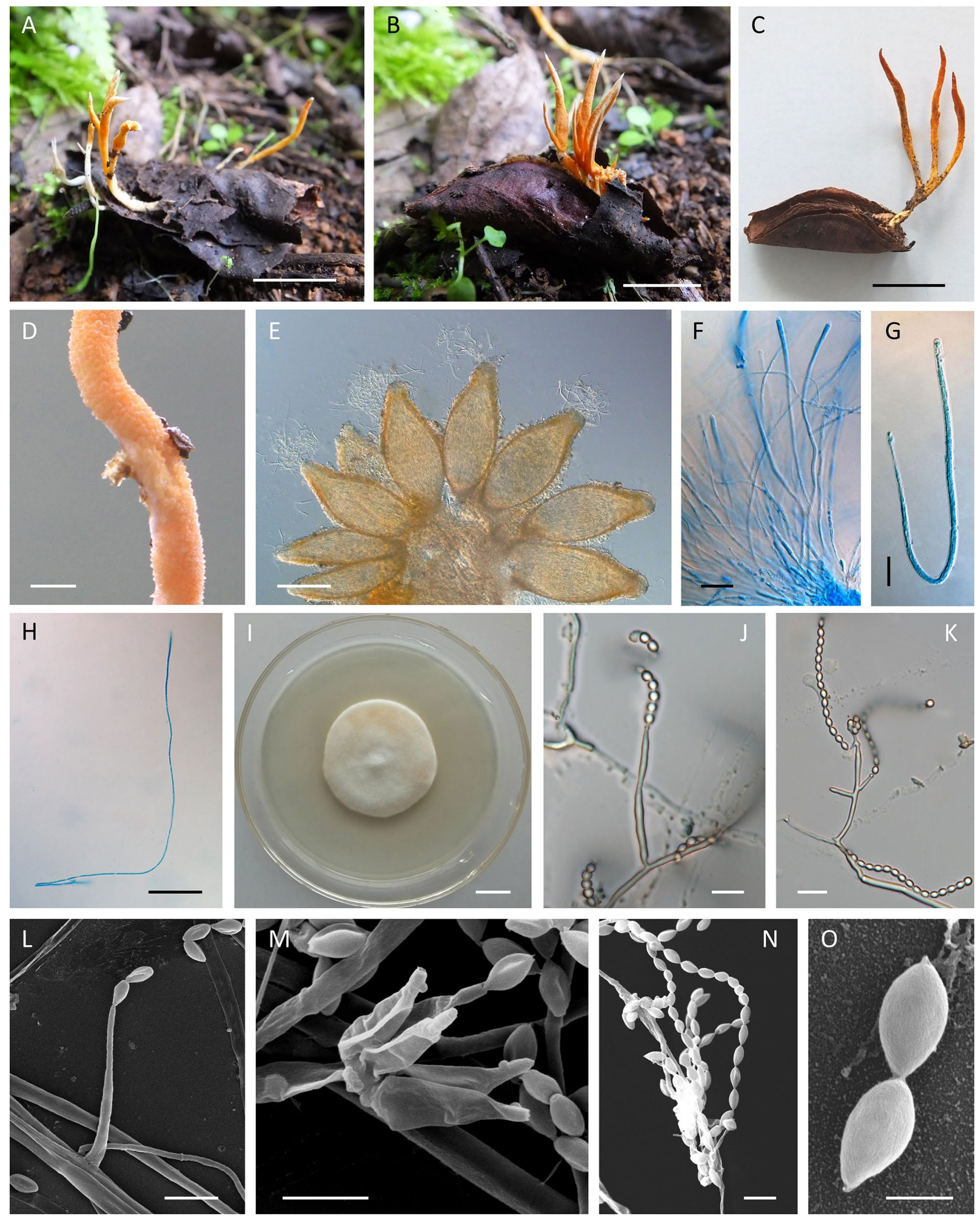

Fig. 18 Samsoniella tortricidae. A-C Stromata arising from the pupae of Tortricidae in cocoons rolled in fallen leaves. D Fertile parts. E Perithecia. F, G Asci. H Ascospore. I Colony on PDA.
J-M Conidiophores and phialides. N, O Conidia in chains. Scale bars: $\mathbf{A}-\mathbf{C}=1 \mathrm{~cm} ; \mathbf{D}=1 \mathrm{~mm} ; \mathbf{E}=200 \mu \mathrm{m} ; \mathbf{F}-\mathbf{H}=20 \mu \mathrm{m} ; \mathbf{I}=1 \mathrm{~cm}$; $\mathbf{J}-\mathbf{N}=5 \mu \mathrm{m} ; \mathbf{O}=2 \mu \mathrm{m}$ 
Sexual morph: Stromata gregarious, arising from insect cocoons rolled in fallen leaves, up to $25-60 \mathrm{~mm}$ long, unbranched or dichotomous. Stipes flexuous, yellowish to orange, cylindrical to clavate, $12-46 \times 1.1-3.0 \mathrm{~mm}$. Fertile parts reddish orange, clavate to subulate, ateral side usually has a longitudinal section without producing perithecia, 5-15×1.2-2.3 mm. Perithecia crowded, superficial, narrowly ovoid to fusiform, $350-468 \times 140-225 \mu \mathrm{m}$. Asci eightspored, hyaline, cylindrical, up to $170-285 \times 2.8-4.0 \mu \mathrm{m}$. Ascus caps hemispherical, 2.2-3.3 $\times 1.4-2.2 \mu \mathrm{m}$. Ascospores hyaline, bola-shaped, septate, $120-235 \times 0.8-1.3 \mu \mathrm{m}$, central part filiform, terminal part narrowly fusiform, do not disarticulate into part-spores. Asexual morph: Isaria-like. Colonies on PDA grow well, 30-36 mm diameter in 14 days at $25^{\circ} \mathrm{C}$, white to pale pink, cottony, sporulating abundantly, reverse orange to reddish-brown. Hyphae smooth-walled, branched, septate, hyaline, 1.1-2.4 $\mu \mathrm{m}$ wide. Conidiophores smooth-walled, cylindrical, solitary, 4.2-12.5×1.4-2.4 $\mu \mathrm{m}$. Phialides verticillate, in whorls of two to five, usually solitary on hyphae, basal portion cylindrical to narrowly lageniform, tapering gradually or abruptly toward the apex; 3.6-42.4 $\mu \mathrm{m}$ long, 1.1-2.6 $\mu \mathrm{m}$ wide at the base, and $0.4-0.9 \mu \mathrm{m}$ wide at the apex. Conidia one-celled, smoothwalled, hyaline, fusiform or oval, 2.1-3.0 $\times 1.3-1.7 \mu \mathrm{m}$, often in chains.

Host: Pupae of Tortricidae (Lepidoptera)

Habitat: On the pupae of Tortricidae (Lepidoptera) in cocoons rolled in fallen leaves.

Distribution: Kunming City, China.

Material examined: CHINA. YUNNAN PROVINCE: Kunming City, Wild Duck Lake Forest Park, on the pupa of Tortricidae (Lepidoptera) in a cocoon rolled in fallen leaves, 12 August 2017, Hong Yu, (YHH 16050, holotype; YFCC 6131, ex-holotype living culture); Ibid., (YHH 15989, paratype; YFCC 6013, ex-paratype living culture); Ibid., (YHH 16064, paratype; YFCC 6142, ex-paratype living culture).

Notes: Samsoniella tortricidae is characterized by gregarious stromata, unbranched or dichotomous, cylindrical to clavate stipes, clavate to subulate fertile parts with reddish orange color, superficial perithecia, narrowly ovoid to fusiform, cylindrical asci with bola-shaped ascospores, and having Isaria-like asexual conidiogenous structure.

Samsoniella tortricidae resembles the phylogenetic sister species $S$. cristata in producing stromata with terminal branches, subulate fertile parts, and Isaria-like asexual conidiogenous structure. However, it differs from $S$. cristata by its gregarious stromata up to $25-60 \mathrm{~mm}$ long, unbranched or dichotomous, white to pale pink cottony colonies, sporulating abundantly, longer phialides (3.6-42.4 $\mu \mathrm{m})$. Ecologically, $S$. tortricidae is parasitic on the pupae of Tortricidae (Lepidoptera) in cocoons rolled in fallen leaves and is very different from $S$. cristata, which is parasitic on the pupae of Saturniidae in cocoons buried in soil.
Samsoniella yunnanensis H. Yu, Y.B. Wang, Y. Wang, D.E. Duan \& Zhu L. Yang, sp. nov.

Mycobank: MB 833119; Fig. 19

Etymology: Named after the location Yunnan Province where the species was collected.

Holotype: YHH 3126

Sexual morph: Undetermined. Asexual morph: Isarialike. Synnemata arising from the insect cocoons, gregarious, flexuous, fleshy, $0.4-1.8 \mathrm{~cm}$ long, with terminal branches of $3-7 \times 1.0-2.0 \mathrm{~mm}$. Stipes clavate to spatulate, orange to pink, about $1 \mathrm{~mm}$ wide, producing a mass of conidia toward the apex of synnemata, powdery and floccose. Conidiophores biverticillate with phialides in whorls of two to seven, cylindrical, 4.0-22.7 $\times 1.5-2.5 \mu \mathrm{m}$. Phialides with a basal portion cylindrical to narrowly lageniform, $4.2-12.1 \times 1.3-2.5 \mu \mathrm{m}$, tapering gradually or abruptly into a narrow neck of $0.5-1.0 \mu \mathrm{m}$ wide. Conidia in chains, one-celled, smoothwalled, hyaline, fusiform or oval, $2.0-2.8 \times 1.2-2.0 \mu \mathrm{m}$. Colonies on PDA fast-growing, $48-50 \mathrm{~mm}$ diameter in 14 days at $25^{\circ} \mathrm{C}$, white, consisting of high mycelial density, loose and hairy, sporulating abundantly, reverse reddishbrown. Hyphae smooth-walled, branched, septate, hyaline, $1.2-2.3 \mu \mathrm{m}$ wide. Conidiophores smooth-walled, cylindrical, solitary or verticillate, $4.2-23.5 \times 1.4-2.3 \mu \mathrm{m}$. Phialides verticillate, in whorls of two to seven, usually solitary on hyphae, basal portion cylindrical to narrowly lageniform, tapering gradually or abruptly toward the apex; $4.5-11.6 \mu \mathrm{m}$ long, 1.2-2.4 $\mu \mathrm{m}$ wide at the base, and 0.6-1.0 $\mu \mathrm{m}$ wide at the apex. Conidia one-celled, smooth-walled, hyaline, fusiform or oval, 2.0-3.3 $\times 1.1-2.2 \mu \mathrm{m}$, often in chains.

Host/Substratum: Pupae of Limacodidae, Cordyceps sp. and Cordyceps cicadae Shing.

Habitat: On the pupae of Limacodidae in cocoons, Cordyceps sp. associated with the pupae of Lepidoptera and $C$. cicadae associated with the nymphs of Cicadidae buried in soil.

Distribution: Kunming City and Shangrila City, China.

Material examined: CHINA. YUNNAN PROVINCE: Diqing Tibetan Autonomous Prefecture, Shangrila City, Hutiaoxia Town, isolated from C. cicadae associated with the nymph of Cicadidae buried in soil, 26 July 2007, YiJian Chen, (YHH 3126, holotype); YFCC 1527, ex-holoype living culture); same location, isolated from $C$. cicadae associated with the nymph of Cicadidae buried in soil, 10 July 2008, Zi-Hong Chen, (YHH 3715; YFCC 1824, living culture); same location, on the pupa of Limacodidae in a cocoon buried in soil, 08 October 2010, Yuan-Bing Wang, (YHH 4520; YFCC 2830, living culture). Kunming City, Wild Duck Lake Forest Park, on Cordyceps sp. associated with the pupa of Lepidoptera buried in soil, 14 August 2018, Yao Wang, (YHH 17053; YFCC 7282, living culture).

Notes: Samsoniella yunnanensis is characterized by gregarious synnemata with terminal branches, clavate to 

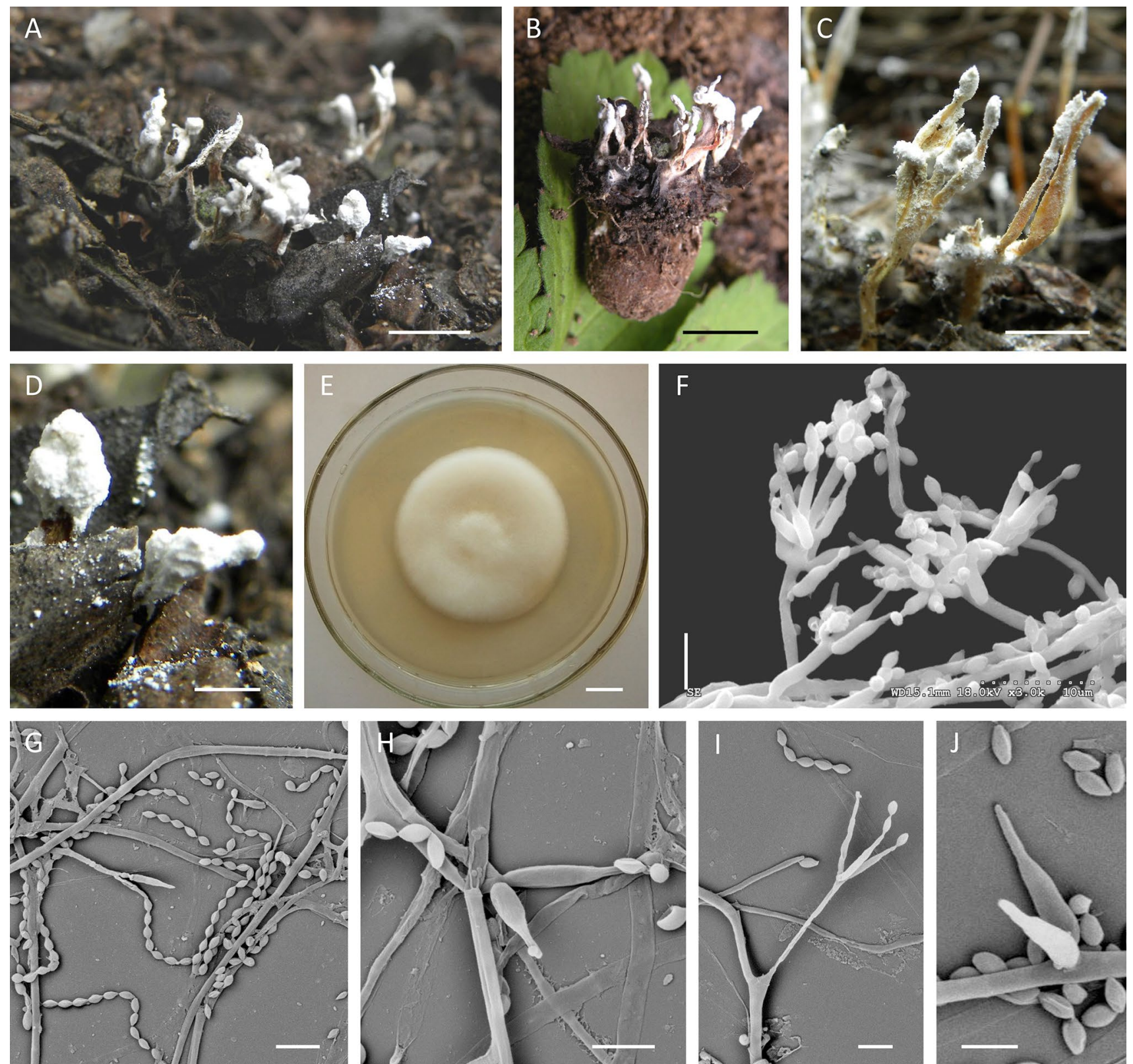

Fig. 19 Samsoniella yunnanensis. A, B Synnemata arising from the pupa of Limacodidae in a cocoon. C, D Stipes producing a white mass of conidia toward the apex. E Colony on PDA. F-I Verticillate phialides on conidiophores. J Verticillate phialides on hypha. Scale bars: $\mathbf{A}-\mathbf{D}=5 \mathrm{~mm} ; \mathbf{E}=1 \mathrm{~cm} ; \mathbf{F}-\mathbf{J}=5 \mu \mathrm{m}$

conidia toward the apex synnemata with terminal branches and Isaria-like asexual conidiogenous structure. Additionally, none of these three fungal sexual morphs have been determined yet. However, it differs from the latter two by its orange to pink stipes, and associations with the pupae of Limacodidae in cocoons, Cordyceps sp. associated with the pupa of Lepidoptera, and $C$. cicadae associated with the nymphs of Cicadidae buried in soil. 

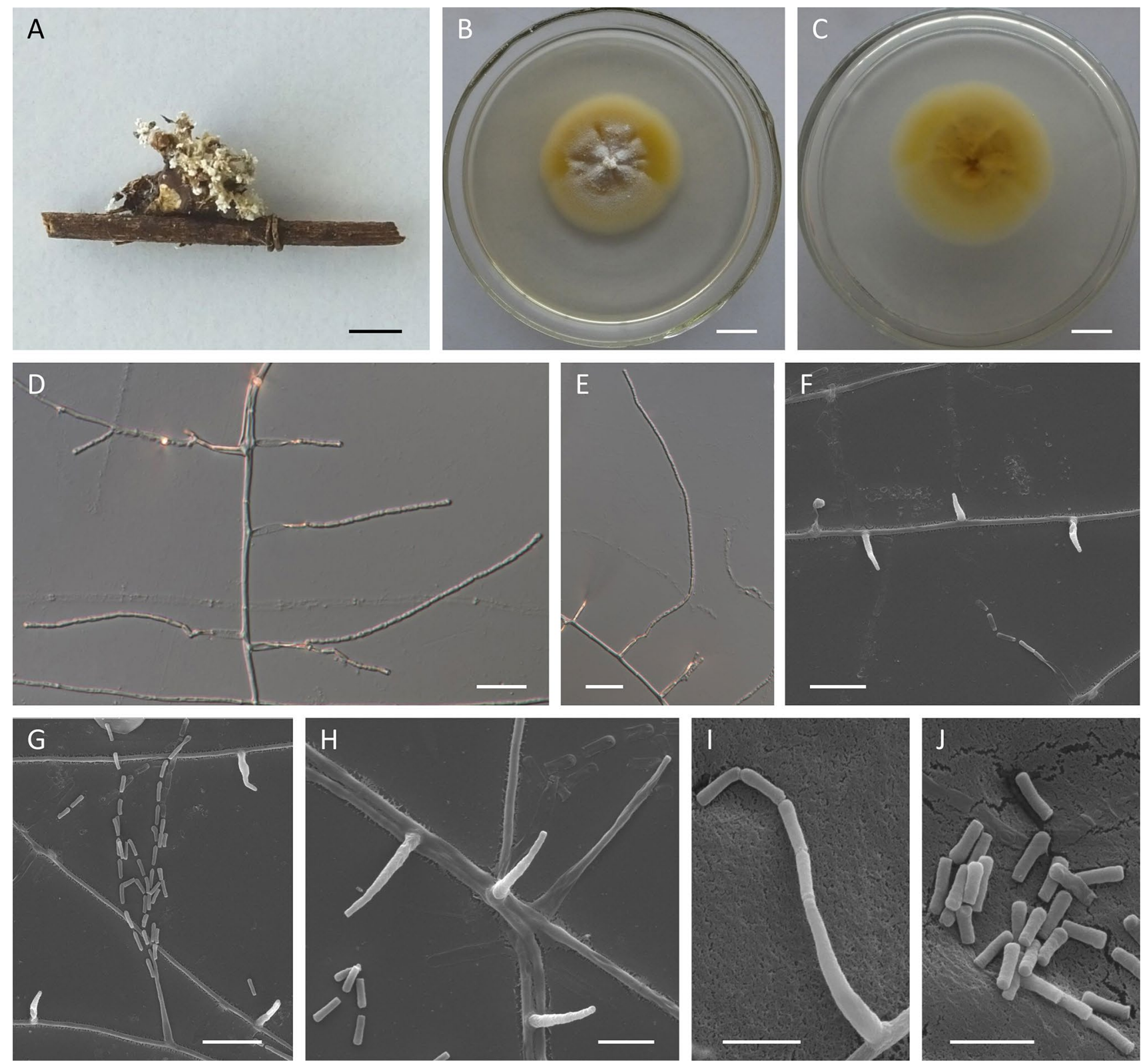

Fig. 20 Simplicillium yunnanense. A Akanthomyces waltergamsii associated with the spider on a dead stem, from which Sim. yunnanense was isolated. B Colony on PDA. C Reverse of colony. D-I

Simplicillium yunnanense H. Yu, Y.B. Wang, Y. Wang \& Zhu L. Yang, sp. nov.

Mycobank: MB 833120; Fig. 20

Etymology: Named after the location Yunnan Province where this species was collected.

Holotype: YHH 16988

Sexual morph: Undetermined. Asexual morph: Colonies on PDA fast-growing, 39-42 mm diameter in 14 days at $25{ }^{\circ} \mathrm{C}$, convex, white to light yellow, with very low mycelial density, producing hyaline droplets on the felty aerial mycelium, generating radially distributed grooves.
Solitary phialides with conidia in chains produced on prostrate aerial hyphae. J Cylindrical conidia. Scale bars: $\mathbf{A}=2 \mathrm{~mm} ; \mathbf{B}, \mathbf{C}=1 \mathrm{~cm}$; $\mathbf{D}-\mathbf{G}=10 \mu \mathrm{m} ; \mathbf{H}-\mathbf{J}=5 \mu \mathrm{m}$

Reverse grayish orange to brown. Hyphae smooth-walled, branched, septate, hyaline, $0.8-1.7 \mu \mathrm{m}$ wide. Phialides produced on prostrate aerial hyphae, solitary, discrete, subulate, tapering gradually toward the apex, 5.8-16.9 $\mu \mathrm{m}$ long, $1.1-1.5 \mu \mathrm{m}$ wide at the base, and $0.6-1.1 \mu \mathrm{m}$ wide at the apex. Conidia one-celled, smooth-walled, hyaline, cylindrical, $2.5-3.4 \times 0.7-1.1 \mu \mathrm{m}$, usually in chains at the phialidic apex.

Substratum: Akanthomyces waltergamsii Mongkols et al. Habitat: On A. waltergamsii associated with the spider on a dead stem. 
Distribution: Kunming City, China.

Material examined: CHINA. YUNNAN PROVINCE: Kunming City, Wild Duck Lake Forest Park, isolated from synnemata of $A$. waltergamsii associated with the spider on a dead stem, 14 August 2018, Yao Wang, (YHH 16988, holotype; YFCC 7133, ex-holotype living culture); Ibid., (YHH 16989; YFCC 7134, living culture).

Notes: Simplicillium yunnanense is characterized by white to light yellow colonies with very low mycelial density, convex, generating radially distributed grooves, solitary phialides, discrete, subulate, produing cylindrical conidia usually in chains at the apex of phialides, and on A. waltergamsii associated with the spider on the dead stem.

In our five-gene phylogenetic analyses (Fig. 1, 2), Sim. yunnanense forms a separate clade in Simplicillium and is likely to be related to Sim. lanosoniveum (J.F.H. Beyma) Zare \& W. Gams and Sim. obclavatum (W. Gams) Zare \& W. Gams. However, it differs from the latter two by its shorter phialides with subulate shape and producing cylindrical conidia usually in chains at the apex of phialides. Simplicillium lanosoniveum and Sim. obclavatum have much narrower and longer phialides, producing respectively oval or ellipsoidal to subcylindrical, obclavate to ellipsoidal conidia, and form respectively globose heads and short imbricate chains at the apex of phialides (Zare and Gams 2001). Ecologically, Sim. yunnanense can be differentiated due to its association with synnemata of $A$. waltergamsii parasitizing the spider.

\section{Discussion}

Many high-level phylogenetic classifications for entomopathogenic fungi have been undertaken, thus more and more available molecular data can be efficiently used to facilitate systematics and evolutionary biology of cordycipitoid fungi (Sung et al. 2007; Chaverri et al. 2008; Kepler et al. 2013, 2014, 2017; Quandt et al. 2014; Maharachchikumbura et al. 2015; Hongsanan et al. 2017; Luangsa-ard et al. 2017; Mongkolsamrit et al. 2018). In this study, we focused on the phylogenetic investigation of the family Cordycipitaceae, with special emphasis on species collected from Yunnan Province, China. Our phylogenetic study supports recognition of the genera Cordyceps, Samsoniella, Lecanicillium and Simplicillium, as previously reported (Kepler et al. 2017; Mongkolsamrit et al. 2018). We proposed two new genera Flavocillium and Liangia, 16 new species and four new combinations in the family Cordycipitaceae.

The genus Flavocillium was erected to accommodate $F$. bifurcatum, $F$. acerosium, $F$. primulinium, and $F$. subprimulinium. Even though Flavocillium is morphologically similar to other Lecanicillium species in conidiophores, phialides and two types of conidia, the genus is sufficiently distinct by possessing yellowish stromata with a furcate terminal branch, contorted fertile parts, and colonies that usually produce pastel yellow pigment (Zare and Gams 2001; Kaifuchi et al. 2013; Huang et al. 2018; Su et al. 2019). In addition, Flavocillium is distinguished from phylogenetically close relatives Engyodontium and Parengyodontium because the latter two genera usually produce white colonies, conidiiferous rachids with denticles on phialides, and terminal fertile regions that are zigzag-shaped (Gams et al. 1984; Tsang et al. 2016). Liangia is established for the new species Lia. sinensis isolated from the cordycipitoid fungus B. yunnanensis. Liangia is more closely related to C. piperis and L. psalliotae clades in the five-gene phylogenetic analyses. However, this genus differs morphologically from $C$. piperis that produces the Verticillium-like anamorph with verticillate conidiophores and phialides, subcylindrical conidia aggregating into heads and conjoined polyhedral crystals (Bischoff and White 2004). Liangia is similar to L. psalliotae in sharing the Lecanicillium-like asexual morph, but it differs from the latter that produces erect conidiophores, relatively short verticillate phialides, short-ellipsoidal conidia formed in heads and octahedral crystals (Zare and Gams 2001). The new genera Flavocillium and Liangia can be distinguished from each other by having distinct morphological characteristics and phylogenetic positions.

The economically and medically significant fungus $P$. hepiali was reexamined and assigned to the genus Samsoniella based on study of the holotype IMM 82-2 and its ex-holotype living culture, as well as seven other samples of $P$. hepiali. The systematic position of $P$. hepiali is most appropriate in the genus Samsoniella. Therefore, the new taxonomic combination $S$. hepiali is proposed for $P$. hepiali. Collections of unknown identity are found to represent nine new species of Samsoniella, which are named S. alpina, $S$. antleroides, $S$. cardinalis, S. cristata, S. kunmingensis, S. lanmaoa, S. ramosa, S. tortricidae and S. yunnanensis. Four new species of Cordyceps are described and named $C$. subtenuipes, $C$. shuifuensis, $C$. chaetoclavata and $C$. cocoonihabita. Two isolations from $A$. waltergamsii associated with the spider on the dead stem represent a new species in the genus Simplicillium, viz. Sim. yunnanense. All of the above species are recognized as new members of the family Cordycipitaceae by well-supported morphological and molecular phylogenetic evidence.

The asexual genus Lecanicillium is typified by L. lecanii with the sexual morph $T$. confragosa and previously contained 32 species (Zare and Gams 2001, 2008; Sukarno et al. 2009; Crous et al. 2018; Huang et al. 2018; Su et al. 2019; Zhou et al. 2018). However, the recent taxonomic revision rejected $L$. lecanii, the type species of Lecanicillium, and considered that it was a synonym of Akanthomyces (Kepler et al. 2017). This treatment seeks to harmonize competing names by principles of priority, recognition of monophyletic 
groups, and the practical usage of the affected taxa. Based on this, Akanthomyces was proposed to be maintained and Lecanicillium was rejected, although the generic name Lecanicillium was still being used thereafter (Crous et al. 2018; Huang et al. 2018; Su et al. 2019; Zhou et al. 2018). Species of different lineages may have similar micromorphological characteristics like those of Lecanicillium in the family Cordycipitaceae. Unfortunately, many species in the Lecanicillium lineage are published with singular gene data, such as ITS sequences. Few multigene sequences are available in online databases. Reconstructing the credible phylogenetic framework of Lecanicillium clades in the family Cordycipitaceae is difficult due to a lack of large-scale multigene sequence sampling. Thus, in this study, the phylogenetic tree of Lecanicillium inferred from ITS sequences includes eight clades, which does not match those of the tree generated from five-gene data because of differentiated available data sampling. To be prudent, we did not make major revisions to the Lecanicillium lineage but only added two genera, Flavocillium and Liangia, based on their monophyly and distinct morphological characteristics in the family Cordycipitaceae. A credible phylogenetic framework of Lecanicillium species, which have not been assigned appropriate generic names, will require more future extensive multigene taxon sampling.

Phylogenetic classifcations of entomopathogenic fungi showed that most diagnostic characteristics used in current classifcations of cordycepitoid fungi (e.g., arrangement of perithecia, ascospores fragmentation, conidiogenous structures, conidial shape and size) are not phylogenetically informative (Sung et al. 2007; Kepler et al. 2013, 2017; Ban et al. 2015; Mongkolsamrit et al. 2018). However, the characteristics that are most consistent with the phylogeny are texture, pigmentation and morphology of the stromata and synnemata. Even so, these macro- and micro-morphological characteristics could aid the identification of Cordyceps, Isaria-like and Lecanicillium-like fungi. Sexual morphs of Cordyceps are characterized by fleshy stromata, red to orange colors, superficial perithecia, asci cylindrical with thickened ascus apex, ascospores usually cylindrical and multiseptate. These are very similar to those of Samsoniella, which mainly have lepidopteran hosts. Previous studies of cordycipitoid fungi as well as our study show that both Samsoniella and Cordyceps species produce similar asexual conidiogenous structures (Samson 1974; Mongkolsamrit et al. 2018). Samsoniella and Cordyceps share similar Isaria-like asexual morphs that produce branched and white to orange synnemata, a dry mass of white to cream conidia on the synnemata, flask-shaped phialides that are produced in whorls, conidia with divergent chains. Therefore, the C. farinosa morphology is not diagnostic and represents a polyphyletic species complex as exemplified by the isolates delimited as S. alboaurantium (G. Sm.) Mongkolsamrit et al. (Kepler et al. 2017; Mongkolsamrit et al. 2018).

A review of the taxonomic history of Cordyceps concluded that Cordyceps is the oldest accepted generic name in the family Cordycipitaceae (Shrestha et al. 2014). Based on the cylindrical shape of stroma, pre-Linnaean literature of the 17th and early eighteenth centuries had recorded $C$. militaris. It is noteworthy that Cordyceps has a much longer history and culture in China. The famous Chinese medicine monograph "Shennong's Materia Medica" (Qin and Han Dynasties, second century BC) recorded the white muscardin silkworms infected by $B$. bassiana as a medicine. The archaeology of Haihun marquis (Western Han Dynasty, first century AD) discovered Cordyceps sp. in He Liu's funerary objects, proving that Cordyceps sp. had been used for health care in China as early as 2075 years ago. The "Mister Lei's Treatise on Processing Drugs" (Southern and Northern Dynasties, 5th Century AD) recorded I. cicadae Miq. as a traditional medicine. Tibetan Materia Medica "Medical King's Drugs for Medicine" (Tibetan Empire, 8th Century AD) recorded $O$. sinensis as a medicine.

In the phylogenetic classification of cordycipitoid fungi, the desire to preserve the term "cordyceps" within the family Ophiocordycipitaceae to reflect the cultural and economic importance of $O$. sinensis was expressed (Sung et al. 2007). This taxonomic revision ultimately benefits humanity, especially in Asia. Samsoniella hepiali (syn. P. hepiali) is also termed "cordyceps", and it is internationally known. Based on the above, we suggest that the Chinese name “鳞翅虫草 属” (Lín Chì Chóng Căo Shǔ), be given to Samsoniella, taking into account the similarity of its morphological and ecological characteristics with Cordyceps and practical usage.

Samsoniella hepiali is a very important fungus to humans, due to its therapeutic effects in cardiovascular, respiratory disorders, immunomodulatory, hyposexuality, hyperglycemia, renal disorder and antitumor conditions (Lou et al. 1986; Huang et al. 1988; Wang and Huang 1988; Dai et al. 1989; Zou and Huang 1993; Xiang et al. 2006; Jiang et al. 2010). The Ministry of Health of the P. R. China issued a new drug certificate (WYZZ2-67 05) in July, 1987 and listed the $S$. hepiali strain Cs-4 as a protected and confidential strain. The product of strain Cs-4, Jinshuibao capsule, was introduced into the market in 1987 . The Ministry of Health of the P. R. China issued File No. 84 on 23 March 2001 and approved S. hepiali mycelia to be used as a stand-alone or a component of health foods (equivalent to dietary supplements in other countries) (Dai et al. 2018b). Thus, $S$. hepiali is widely used as a medicinal and edible cordycipitoid fungus, creating an annual economic value of approximately 10 billion RMB in China. In addition to the Jinshuibao capsule, over 260 healthcare products have been developed with $S$. hepiali as a raw material. Its therapeutic 
effects have been demonstrated and are now widely recognized by doctors and patients. Many companies have put these products into Chinese markets and globally exported them to nearly 80 countries or regions as medicine and dietary supplements, including northeastern and southeastern Asia, the United States of America, Canada, Australia, New Zealand and other countries (Dai et al. 2018b). Samsoniella hepiali is economically, medicinally and culturally important, and share the morphologically and ecologically similar characteristics with Cordyceps. Based on its significant contribution as "cordyceps", here we strongly suggest that the Chinese name “蝙蝠蛾虫草” (Biān Fú É Chóng Căo), be given to this cordycipitoid fungus, which will allow for the convenient and unambiguous communication among the biomedical and health industries of China.

Acknowledgements We are grateful to the Institute of Chinese Materia Medica, China Academy of Chinese Medical Sciences for providing the holotype material (CHICMM) IMM 82-2 and its ex-holotype living culture of Paecilomyces hepiali. Thanks are due to Prof. Zong-Qi Liang for his valuable suggestions about the taxonomy of $P$. hepiali and its related taxa. We thank Prof. Zhuo Zhang for providing a strain of P. hepiali. We are grateful to Mr. Run-De Yang, Prof. Yu-Ling Li, Dr. Zhong-Lin Yang, Dr. Feng Yuan, Mr. Kun Yang, Mr. Chang-Kui Wu, Mr. Rui Guo, Mr. Xian-Yan Ma, Mr. Tao Sun, Mr. Yun-Peng Sun, Mrs. Xue-Yuan Jia, Mr. Da-Kun Sun, Mr. Jian Chen, Mr. Tao Shen, Mr. Xi-Jun Dang, Prof. Wen-Ju Zhang, Dr. Ya-Nan Wang, Dr. Li Wang, Mr. Van-Minh Dao, Prof. Jing-Yu Zhang, Prof. Hong-Ren Yang, Mr. Wan-Wei Xu, Mr. Zi-Hao Liu, Mr. Yue-Tin Li and Mr. Neng Li for providing collections. We are very grateful to Mrs. Jun-Yuan Yang, Ms. Jiao Cai, Mr. Dong Wang, Mrs. Si-Qi Chen, Mrs. Yan-Fang Liu, Ms. Chen-Xin Chang, Ms. Zi-Jiao Wang, Ms. Yu-Feng Ma, Ms. Manzilamu Zaman, Ms. Juan Li, Mr. Yin-Long Yang, Ms. Dong-Yi Cao, Ms. JingJing Tong and Ms. Yong-Yu Li for isolating strains. This work was jointly funded by the National Natural Science Foundation of China [31870017, 31760011], the Ministry of Science and Technology of the People's Republic of China [KC1610530], the China Postdoctoral Science Foundation [2017M613017], the Department of Science and Technology of Yunnan Province [2018IA075, 2018FY001(-006)], the Biodiversity Survey and Assessment Project of the Ministry of Ecology and Environment, China [2019HJ2096001006], the Biodiversity Investigation, Observation and Assessment Program (2019-2023) of the Ministry of Ecology and Environment of China, and the Yunnan University's Research Innovation Fund for Graduate Students [YDY17100].

Open Access This article is licensed under a Creative Commons Attribution 4.0 International License, which permits use, sharing, adaptation, distribution and reproduction in any medium or format, as long as you give appropriate credit to the original author(s) and the source, provide a link to the Creative Commons licence, and indicate if changes were made. The images or other third party material in this article are included in the article's Creative Commons licence, unless indicated otherwise in a credit line to the material. If material is not included in the article's Creative Commons licence and your intended use is not permitted by statutory regulation or exceeds the permitted use, you will need to obtain permission directly from the copyright holder. To view a copy of this licence, visit http://creativecommons.org/licenses/by/4.0/.

\section{References}

Ban S, Sakane T, Nakagiri A (2015) Three new species of Ophiocordyceps and overview of anamorph types in the genus and the family Ophiocordyceptaceae. Mycol Prog 14:1017

Bainer G (1907) Mycotheque del' Ecole de Pharmacie XI. Paecilomyces, Genre Noveau de Mucedinees. Bull Soc Mycol France 23:26-27

Bischoff JF, White JF (2004) Torrubiella piperis sp. nov. (Clavicipitaceae, Hypocreales), a new teleomorph of the Lecanicillium complex. Stud Mycol 50:89-94

Bischoff JF, Rehner SA, Humber RA (2006) Metarhizium frigidum sp. nov.: a cryptic species of $M$. anisopliae and a member of the $M$. flavoviride Complex. Mycologia 98(5):737-745

Chaverri P, Liu M, Hodge KT (2008) A monograph of the entomopathogenic genera Hypocrella, Moelleriella, and Samuelsia gen. nov. (Ascomycota, Hypocreales, Clavicipitaceae), and their aschersonia-like anamorphs in the Neotropics. Stud Mycol 60:1-66

Chen ZH, Chen K, Dai YD, Zheng Y, Wang YB, Yang XN, Yu H, Yang YM, Xu L (2019) Beauveria species diversity in the Gaoligong Mountains of China. Mycol Prog 18(7):933-943

Crous PW, Wingfield MJ, Burgess TI, Hardy GEStJ, Gené J, Guarro J, Baseia IG, García D, Gusmão LFP, Souza-Motta CM, Thangave R, Adamčík S, Barili A, Barnes CW, Bezerra JDP, Bordallo JJ, Cano-Lira JF, de Oliveira RJV, Ercole E, Hubka V, IturrietaGonzález I, Kubátová A, Martín MP, Moreau PA, Morte A, Ordoñez ME, Rodríguez A, Stchige AM, Vizzini A, Abdollahzadeh J, Abreu VP, Adamčíková K, Albuquerque GMR, Alexandrova AV,Álvarez Duarte E, Armstrong-Cho C, Banniza S, Barbosa RN, Bellanger JM, Bezerra JL, Cabral TS, Caboň M, Caicedo E, Cantillo T, Carnegie AJ, Carmo LT, Castañeda-Ruiz RF, Clement CR, Čmoková A, Conceição LB, Cruz RHSF, Damm U, da Silva BDB, da Silva GA, da Silva RMF, de A. Santiago ALCM, de Oliveira LF, de Souza CAF, Déniel F, Dima B, Dong G, Edwards J, Félix CR, Fournier J, Gibertoni TB, Hosaka K, Iturriaga T, Jadan M, Jany JL, Jurjević Ž, Kolařík M, Kušan I, Landell MF, Leite Cordeiro TR, Lima DX, Loizides M, Luo S, Machado AR, Madrid H, Magalhães OMC, Marinho P, Matočec N, Mešić A, Miller AN, Morozova OV, Neves RP, Nonaka K, Nováková A, Oberlies NH, Oliveira-Filho JRC, Oliveira TGL, Papp V, Pereira OL, Perrone G, Peterson SW, Pham THG, Raja HA, Raudabaugh DB, Řehulka J, Rodríguez-Andrade E, Saba M, Schauflerová A, Shivas RG, Simonini G, Siqueira JPZ, Sousa JO, Stajsic V, Svetasheva T, Tan YP, Tkalčec Z, Ullah S, Valente P, Valenzuela-Lopez N, Abrinbana M, Viana Marques DA, Wong PTW, Xavier de Lima V, Groenewald JZ (2018) Fungal Planet description sheets: 716-784. Persoonia 40:240-393

Dai RQ, Lan JL, Chen WH, Li XM, Chen QT, Shen CY (1989) Research on Paecilomyces hepiali Chen et Dai, sp. nov. Acta Agric Univ Pekin 15(2):221-224

Dai RQ, Li XM, Shao AJ, Lin SF, Lan JL, Chen WH, Shen CY (2008) Nomenclatural validation of Paecilomyces hepiali. Mycosystema 27(5):641-644

Dai RQ, Shen CY, Li XM, Lan JL, Lin SF, Shao AJ (2018a) Discussion on related problems of model species of fungus: Paecilomyces hepialid. China J Chin Mater Med 42(19):3843-3846

Dai RQ, Shen CY, Li XM, Lan JL, Lin SF, Shao AJ (2018b) Response to neotypification of Paecilomyces hepiali (Hypocreales) (Wang $\&$ al., 2015). Taxon 67 (4):784-786

Dayal R, Barron GL (1970) Verticillium psalliotae as a parasite of Rhopalomyces. Mycologia 62(4):826-830

Darriba D, Taboada GL, Doallo R, Posada D (2012) jModelTest 2: more models, new heuristics and parallel computing. Nat Methods 9:772 
Gams W, de Hoog GS, Samson RA, Evans HC (1984) The hyphomycete genus Engyodontium: a link between Verticillium and Aphanocladium. Persoonia 12:135-147

Hongsanan S, Maharachchikumbura SSN, Hyde KD, Samarakoon MC, JeewonR ZQ, Al-Sadi AM, Bahkali AH (2017) An updated phylogeny of Sordariomycetes based on phylogenetic and molecular clock evidence. Fungal Divers 84(1):25-41

Huang MM, Zhang JF, Pang L, Jiang Z, Wang DW (1988) Studies on immunopharmacology of Cordyceps (Fr.) Link IV. Observations on the immunosuppressive activity of artificial substance of Paecilomyces hapiali Chen. Acta Univ Med Tongji 5:329-331

Huang SK, Maharachchikumbura SSN, Jeewon R, Bhat DJ, Phookamsak R, Hyde KD, Al-Sadi AM, Kang JC (2018) Lecanicillium subprimulinum (Cordycipitaceae, Hypocreales), a novel species from Baoshan, Yunnan. Phytotaxa 348(2):099-108

Humber RA, Rocha LFN, Inglis PW, Kipnis A, Luz C (2013) Morphology and molecular taxonomy of Evlachovaea-like fungi, and the status of this unusual conidial genus. Fungal Biol 117:1-12

Hyde KD, Tennakoon DS, Jeewon R, Bhat DJ, Maharachchikumbura SSN, Rossi W, Leonardi M, Lee HB, Mun HY, Houbraken J, Nguyen TTT, Jeon SJ, Frisvad JC, Wanasinghe DN, Lücking R, Aptroot A, Caceres MES, Karunarathna SC, Hongsanan S, Phookamsak R, de Silva NI, Thambugala KM, Jayawardena RS, Senanayake IC, Boonmee S, Chen J, Luo ZL, Phukhamsakda C, Pereira OL, Abreu VP, Rosado AWC, Buyck B, Randrianjohany E, Hofstetter V, Gibertoni TB, da Silva Soares AM, Plautz HL Jr, Pontes Sotão HM, Xavier WKS, Bezerra JDP, de Oliveira TGL, de Souza-Motta CM, Magalhães OMC, Bundhun D, Harishchandra D, Manawasinghe IS, Dong W, Zhang SN, Bao DF, Samarakoon MC, Pem D, Karunarathna A, Lin CG, Yang J, Perera RH, Kumar V, Huang SK, Dayarathne MC, Ekanayaka AH, Jayasiri SC, Xiao YP, Konta S, Niskanen T, Liimatainen K, Dai YC, Ji XH, Tian XM, Mešić A, Singh SK, Phutthacharoen K, Cai L, Sorvongxay T, Thiyagaraja V, Norphanphoun C, Chaiwan N, Lu YZ, Jiang HB, Zhang JF, Abeywickrama PD, Aluthmuhandiram JVS, Brahmanage RS, Zeng M, Chethana T, Wei DP, Réblová M, Fournier J, Nekvindová J, Barbosa RN, Felinto dos Santos JE, Oliveira NT, Li GJ, Ertz D, Shang QJ, Phillips AJL, Kuo CH, Camporesi E, Bulgakov TS, Lumyong S, Jones EBG, Chomnunti P, Gentekaki E, Bungartz F, Zeng XY, Fryar S, Tkalčec Z, Liang JM, Li GS, Wen TC, Singh PN, Gafforov Y, Promputtha I, Yasanthika E, Goonasekara ID, Zhao RL, Zhao Q, Kirk PM, Liu JK, Yan JY, Mortimer PE, Xu JC, Doilom MW (2019) Fungal diversity notes 1036-1150: taxonomic and phylogenetic contributions on genera and species of fungal taxa. Fungal Divers 96:1-242

Hywel-Jones NL (2002) Multiples of eight in Cordyceps ascospores. Mycol Res 106:2-3

Jiang L, Bao HY, Yang M (2010) Antitumor activity of a petroleum ether extract from Paecilomyces hepiali mycelium. Acta Edulis Fungi 17:58-60

Johnson D, Sung GH, Hywel-Jones NL, Luangsa-Ard JJ, Bischoff JF, Kepler RM, Spatafora JW (2009) Systematics and evolution of the genus Torrubiella (Hypocreales, Ascomycota). Mycol Res 113:279-289

Kaifuchi S, Nonaka K, Mori M, Shiomi K, Ômura S, Masuma R (2013) Lecanicillium primulinum, a new hyphomycete (Cordycipitaceae) from soils in the Okinawa's main island and the Bonin Islands, Japan. Mycoscience 54:291-296

Kepler RM, Ban S, Nakagiri A, Bischoff JF, Hywel-Jones NL, Owensby CA, Spatafora JW (2013) The phylogenetic placement of hypocrealean insect pathogens in the genus Polycephalomyces: an application of one fungus one name. Fungal Biol 117(9):611-622

Kepler RM, Humber RA, Bischoff JF, Rehner SA (2014) Clarifcation of generic and species boundaries for Metarhizium and related fungi through multigene phylogenetics. Mycologia 106(4):811-829
Kepler RM, Luangsa-ard JJ, Hywel-Jones NL, Quandt CA, Sung GH, Rehner SA, Aime MC, Henkel TW, Sanjuan T, Zare R, Chen M, Li Z, Rossman AY, Spatafora JW, Shrestha B (2017) A phylogenetically-based nomenclature for Cordycipitaceae (Hypocreales). IMA Fungus 8(2):335-353

Kobayasi Y, Shimizu D (1960) Monographic studies of Cordyceps 1. Group parasitic on Elaphomyces. Bull Natl Sci Mus Tokyo 5:69-85

Kobayasi Y (1981) Revision of the genus Cordyceps and its allies 1. Bull Natl Sci Mus Tokyo Ser B 7(1):1-13

Kobayasi Y (1982) Keys to the taxa of the genera Cordyceps and Torrubiella. Trans Mycol Soc Japan 23:329-364

Kobayasi Y, Shimizu D (1982) Cordyceps species from Japan 5. Bull Natl Sci Mus Tokyo Ser B 8(4):111-123

Kobayasi Y, Shimizu D (1983) Cordyceps species from Japan 6. Bull Natl Sci Mus Tokyo Ser B 9(1):2-21

Lanfear R, Calcott B, Ho SYW, Guindon S (2012) Partitionfinder: combined selection of partitioning schemes and substitution models for phylogenetic analyses. Mol Biol Evol 29(2):1695-1701

Larkin MA, Blackshields G, Brown NP, Chenna R, McGettigan PA, McWilliam H, Valentin F, Wallace IM, Wilm A, Lopez R, Thompson JD, Gibson TJ, Higgins DG (2007) Clustal W and Clustal X version 2.0. Bioinformatics 23(21):2947-2948

Letunic I, Bork P (2019) Interactive Tree Of Life (iTOL) v4: recent updates and new developments. Nucleic Acids Res 47(W1):W256-W259

Liu ZY, Liang ZQ, Whalley AJS, Yao YJ, Liu AY (2001) Cordyceps brittlebankisoides, a new pathogen of grubs and its anamorph, Metarhizium anisopliae var. majus. J Invertebr Pathol 78:178-182

Luangsa-ard JJ, Hywel-Jones NL, Samson RA (2004) The order level polyphyletic nature of Paecilomyces sensu lato as revealed through 18S-generated rRNA phylogeny. Mycologia 96(4):773-780

Luangsa-ard JJ, Hywel-Jones NL, Manoch L, Samson RA (2005) On the relationships of Paecilomyces sect. Isarioidea species. Mycol Res 109(5):581-589

Luangsa-ard JJ, Houbraken J, van Doorn T, Hong SB, Borman AM, Hywel-Jones NL, Samson RA (2011) Purpureocillium, a new genus for the medically important Paecilomyces lilacinus. FEMS Microbiol Lett 321(2):141-149

Luangsa-Ard JJ, Mongkolsamrit S, Thanakitpipattana D, Khonsanit A, Tasanathai K, Noisripoom W, Humber RA (2017) Clavicipitaceous entomopathogens: new species in Metarhizium and a new genus Nigelia. Mycol Prog 16(4):369-391

Lou YQ, Liao XM, Lu YC (1986) Cardiovascular pharmacological studies of ethanol extracts of Cordyceps mycelia and Cordyceps fermentation solution. Chin Tradit Herbal Drugs 17:17-21

Li DD, Zhang GD, Huang LD, Wang YB, Yu H (2019) Complete mitochondrial genome of the important entomopathogenic fungus Cordyceps tenuipes (Hypocreales, Cordycipitaceae). Mitochondrial DNA B 4(1):1329-1331

Li ZZ, Li CR, Huang B, Fan MZ (2001) Discovery and demonstration of the telemorph of Beauveria bassiana, an important entomogenous fungus. Chin Sci Bull 46(6):470-473

Liang ZQ (1983) A record and description on Cordyceps pruinosa Petch and its conidial state. J Guizhou Agric Coll 2:72-80

Liang ZQ (1991) Verification and identification of the anomorph of Cordyceps pruinosa Petch. Acta Mycol Sin 10(2):104-107

Liang ZQ (2007) Flora fungorum sinicorum vol 32 Cordyceps, vol 32. Science Press, Beijing

Maharachchikumbura SSN, Hyde KD, Jones EBG, McKenzie EHC, Huang SK, Abdel-Wahab MA, Daranagama DA, Dayarathne M, D'souza MJ, Goonasekara ID, Hongsanan S, Jayawardena RS, Kirk PM, Konta S, Liu JK, Liu ZY, Norphanphoun C, Pang KL, Perera RH, Senanayake IC, Shang QJ, Shenoy BD, Xiao YP, Bahkali AH, Kang JC, Somrothipol S, Suetrong S, Wen TC, Xu 
JC (2015) Towards a natural classification and backbone tree for Sordariomycetes. Fungal Divers 72:199-301

Mains EB (1949) New species of Torrubiella, Hirsutella and Gibellula. Mycologia 41:303-310

Mains EB (1958) North American entomogenous species of Cordyceps. Mycologia 50:169-222

Mongkolsamrit S, Noisripoom W, Thanakitpipattana D, Wutikhun T, Spatafora JW, Luangsa-ard JJ (2018) Disentangling cryptic species with isaria-like morphs in Cordycipitaceae. Mycologia 110(1):230-257

Petch T (1924) Studies in entomogenous fungus. IV. Some Ceylon Cordyceps. Trans Br Mycol Soc 10:28-45

Quandt CA, Kepler RM, Gams W, Araújo JPM, Ban S, Evans HC, Hughes D, Humber R, Hywel-Jones NL, Li ZZ, Luangsa-ard JJ, Rehner SA, Sanjuan T, Sato H, Shrestha B, Sung GH, Yao YJ, Zare R, Spatafora JW (2014) Phylogenetic-based nomenclatural proposals for Ophiocordycipitaceae (Hypocreales) with new combinations in Tolypocladium. IMA Fungus 5(1):121-134

Rehner SA, Samuels GJ (1994) Taxonomy and phylogeny of Gliocladium analysed from nuclear large subunit ribosomal DNA sequences. Mycol Res 98(6):625-634

Ronquist F, Huelsenbeck JP (2003) MrBayes 3: Bayesian phylogenetic inference under mixed models. Bioinformatics 19(12):1572-1574

Rossman AY, Samuels GJ, Rogers JS, Lowen R (1999) Genera of Bionectriaceae, Nectriaceae and Hypocreaceae (Hypocreales, Ascomycetes). Stud Mycol 42:1-248

Samson RA, Evans HC (1974) Notes on entomogenous fungi from Ghana. II. The genus Akanthomyces. Acta Bot Neerl 23:28-35

Samson RA (1974) Paecilomyces and some allied hyphomycetes. Stud Mycol 6:1-119

Shrestha B, Tanaka E, Han JG, Oh J, Han SK, Lee KH, Sung GH (2014) A brief chronicle of the genus Cordyceps Fr., the oldest valid genus in Cordycipitaceae (Hypocreales, Ascomycota). Mycobiology 42(2):93-99

Spatafora JW, Quandt CA, Kepler RM, Sung GH, Shrestha B, HywelJones NL, Luangsa-ard JJ (2015) New 1F1N species combinations in Ophiocordycipitaceae (Hypocreales). IMA Fungus 6(2):357-362

Stamatakis A (2006) RAxML-VI-HPC: maximum likelihood-based phylogenetic analyses with thousands of taxa and mixed models. Bioinformatics 22(21):2688-2690

Su CH, Wang HH (1986) Phytocordyceps, a new genus of the Clavicipitaceae. Mycotaxon 26:337-344

Su L, Zhu H, Guo YX, Du XP, Guo JG, Zhang L, Qin C (2019) Lecanicillium coprophilum (Cordycipitaceae, Hypocreales), a new species of fungus from the feces of Marmota monax in China. Phytotaxa 387:55-62

Sukarno N, Kurihara Y, Ilyas M, Mangunwardoyo W, Yuniarti E, Sjamsuridzal W, Park JY, Saraswati R, Inaba S, Widyastuti Y, Ando K, Harayama S (2009) Lecanicillium and Verticillium species from Indonesia and Japan including three new species. Mycoscience 50(5):369-379

Sung GH, Hywel-Jones NL, Sung JM, Luangsa-Ard JJ, Shrestha B, Spatafora JW (2007) Phylogenetic classification of Cordyceps and the clavicipitaceous fungi. Stud Mycol 57:5-59

Swofford DL (2002) PAUP*: Phylogenetic analysis using parsimony (*and other methods), Version 4. Sinauer Associates, Sunderland

Tamura K, Stecher G, Peterson D, Filipski A, Kumar S (2013) MEGA6: molecular evolutionary genetics analysis version 6.0. Mol Biol Evol 30(12):2725-2729

Treschew D (1941) The Verticillium diseases of cultivated mushrooms. Dansk bot Ark 11(1):1-31

Tsang CC, Chan JFW, Pong WM, Chen JHK, Ngan AHY, Cheung M, Lai CKC, Tsang DNC, Lau SKP, Woo PCY (2016) Cutaneous hyalohyphomycosis due to Parengyodontium album gen. et comb. Nov. Med Mycol 54:699-713
Turland NJ, Wiersema JH, Barrie FR, Greuter W, Hawksworth DL, Herendeen PS, Knapp S, Kusber WH, Li DZ, Marhold K, May TW, McNeill J, Monro AM, Prado J, Price MJ, Smith GF (eds.) (2018) International Code of Nomenclature for algae, fungi, and plants (Shenzhen Code) adopted by the Nineteenth International Botanical Congress Shenzhen, China, July 2017. [Regnum Vegetabile no. 159.] Glashütten: Koeltz Botanical Books.

Vilgalys R, Hester M (1990) Rapid genetic identification and mapping of enzymatically amplified ribosomal DNA from several Cryptococcus species. J Bacteriol 172(8):4238-4246

Wang DW, Huang MM (1988) Studies on immunopharmacology of Cordyceps (Fr.) Link V. Influence of artificial fermentative substance of Paecilomyces hapiali Chen on the function of T cell and its subgroup in mice. Acta Univ Med Tongji 5:332-334

Wang YB, Yu H, Dai YD, Wu CK, Zeng WB, Yuan F, Liang ZQ (2015a) Polycephalomyces agaricus, a new hyperparasite of Ophiocordyceps sp. infecting melolonthid larvae in southwestern China. Mycol Prog 14:70

Wang YB, Yu H, Dai YD, Chen ZH, Zeng WB, Yuan F, Liang ZQ (2015b) Polycephalomyces yunnanensis (Hypocreales), a new species of Polycephalomyces parasitizing Ophiocordyceps nutans and stink bugs (hemipteran adults). Phytotaxa 208:034-044

White TJ, Bruns TD, Lee SB, Taylor JW (1990) Amplification and direct sequencing of fungal ribosomal RNA genes for phylogenetics. In: Innis MA, Gelfand DH, Sninsky JJ, White TJ (eds) PCR protocols: a guide to methods and applications. Academic, New York, pp 315-322

Yan JQ, Bau T (2015) Cordyceps ningxiaensis sp. nov., a new species from dipteran pupae in Ningxia Hui Autonomous Region of China. Nova Hedwigia 100:251-258

Yang JK, Huang XW, Tian BY, Sun H, Duan JX, Wu WP, Zhang KQ (2005) Characterization of an Extracellular Serine Protease Gene from the Nematophagous Fungus Lecanicillium psalliotae. Biotechnol Lett 27(17):1329-1334

Xiang M, Tang J, Chu T, Zhang CL, Zou XL (2006) Hypoglycemic effect and mechanism study on streptozocin induced diabetes in mice by Paecilomyces hepiali Chen mycelium. Chin J Hosp Pharm 26:556-559

Yang ZL, Yu H, Chen ZH, Wang YB (2012) Study on the Biological and Ecological Habits of Populations of Cordyceps militaris in Middle of Yunnan Edible Fungi of China. Edible Fungi China 30(5):43-47

Zhang ZF, Liu F, Zhou X, Liu X, Liu SJ, Cai L (2017) Culturable mycobiota from Karst caves in China, with descriptions of 20 new species. Persoonia 39(1):1-31

Zare R, Gams W (2001) A revision of Verticillium section Prostrata. IV. The genera Lecanicillium and Simplicillium gen. nov. Nova Hedwigia 73(1):1-50

Zare R, Gams W (2008) A revision of the Verticillium fungicola species complex and its affinity with the genus Lecanicillium. Mycol Res 112(7):811-824

Zare R, Gams W (2016) More white verticillium-like anamorphs with erect conidiophores. Mycol Prog 15:993-1030

Zhou YM, Zhi JR, Ye M, Zhang ZY, Yue WB, Zou X (2018) Lecanicillium cauligalbarum sp. nov. (Cordycipitaceae, Hypocreales), a novel fungus isolated from a stemborer in the Yao Ren National Forest Mountain Park. MycoKeys 43:59-74

Zou WP, Huang MM (1993) Primary studies on the mechanism of Paecilomyces hepiali Chen against the rejection reaction. Acta Univ Med Tongji 22:282-284 


\section{Affiliations}

\section{Yuan-Bing Wang ${ }^{1,2,3} \cdot$ Yao Wang ${ }^{1,2,4} \cdot$ Qi Fan $^{1,2} \cdot$ Dong-E Duan $^{1,2} \cdot$ Guo-Dong Zhang ${ }^{1,2,3} \cdot$ Ru-Qin Dai ${ }^{6}$.

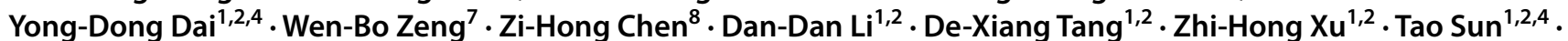 Thi-Tra Nguyen ${ }^{2} \cdot$ Ngoc-Lan Tran $^{9} \cdot$ Van-Minh Dao ${ }^{9} \cdot$ Can-Ming Zhang ${ }^{10} \cdot$ Luo-Dong Huang $^{1} \cdot$ Yong-Jun Liu ${ }^{11}$. Xiao-Mei Zhang ${ }^{1,2,3,12} \cdot$ Da-Rong Yang ${ }^{13} \cdot$ Tatiana Sanjuan $^{14} \cdot$ Xing-Zhong Liu ${ }^{15} \cdot$ Zhu L. Yang ${ }^{4,5} \cdot$ Hong Yu $^{1,2}$}

1 Yunnan Herbal Laboratory, School of Ecology and Environmental Science, Yunnan University, Kunming 650091, China

2 The International Joint Research Center for Sustainable Utilization of Cordyceps Bioresources in China and Southeast Asia, Yunnan University, Kunming 650091, China

3 The Research Center of Cordyceps Development and Utilization of Kunming, Yunnan Herbal Biotech Co. Ltd., Kunming 650106, China

4 CAS Key Laboratory for Plant Diversity and Biogeography of East Asia, Kunming Institute of Botany, Chinese Academy of Sciences, Kunming 650201, China

5 Yunnan Key Laboratory for Fungal Diversity and Green Development, Kunming 650201, China

6 Institute of Chinese Materia Medica, China Academy of Chinese Medical Sciences, Beijing 100700, China

7 College of Evironment and Resources, Wenshan University, Wenshan 663099, China
8 Institute of Biological Resources of Gaoligong Mountains, Baoshan University, Baoshan 678000, China

9 Institute of Regional Research and Development, Ministry of Science and Technology, Hanoi, Vietnam

10 YiKangBao Biotech Co., Ltd, Shangri-La 674400, China

11 School of Chemical Science and Engineering, Yunnan University, Kunming 650091, China

12 College of Basic Medicine, Yunnan University of Chinese Medicine, Kunming 650500, China

13 Key Laboratory of Tropical Forest Ecology, Xishuangbanna Tropical Botanical Garden, Chinese Academy of Sciences, Kunming 650223, China

14 Laboratorio de Taxonomíay Ecología de Hongos, Universidad de Antioquia, calle 67 No. 53-12 108, A.A. 1226, Medellin, Colombia

15 State Key Laboratory of Mycology, Institute of Microbiology, Chinese Academy of Sciences, Beijing 100101, China 